\title{
A Universal Allosteric Mechanism for G Protein Activation.
}

Kevin M. Knight ${ }^{1}$, Soumadwip Ghosh ${ }^{2}$, Sharon L. Campbell ${ }^{3}$, Tyler J. Lefevre ${ }^{4}$, Reid H. J. Olsen $^{1}$, Alan V. Smrcka ${ }^{4}$, Natalie H. Valentin ${ }^{1}$, Guowei Yin ${ }^{3}$, Nagarajan Vaidehi2, ${ }^{2}$, and Henrik G. Dohlman ${ }^{1,5, *}$

${ }^{1}$ Department of Pharmacology, University of North Carolina at Chapel Hill, Chapel Hill, NC 27599, USA

${ }^{2}$ Department of Computational and Quantitative Medicine, Beckman Research Institute of the City of Hope, Duarte, CA 91010, USA

${ }^{3}$ Department of Biochemistry and Biophysics, University of North Carolina at Chapel Hill, Chapel Hill, NC 27599, USA

${ }^{4}$ Department of Pharmacology, University of Michigan, Ann Arbor, MI 48109, USA

${ }^{5}$ Lead contact

* Corresponding authors:

Henrik G. Dohlman hdohlman@med.unc.edu 919-843-6894

Nagarajan Vaidehi NVaidehi@coh.org 626-301-8408 


\section{SUMMARY}

$\mathrm{G}$ proteins play a central role in signal transduction and pharmacology. Signaling is initiated by cell-surface receptors, which promote GTP binding and the dissociation of G $\alpha$ from the G $\beta \gamma$ subunits. Structural studies have revealed the molecular basis for subunit association with receptors, RGS proteins and downstream effectors. In contrast, the mechanism of subunit dissociation is poorly understood. We used cell signaling assays, MD simulations, biochemistry and structural analysis to identify a conserved network of amino acids that dictates subunit release. In the presence of the terminal phosphate of GTP, a glycine forms a polar network with an arginine and glutamate, putting torsional strain on the subunit binding interface. This " $G-R-E$ motif" secures GTP and, through an allosteric link, discharges the G $\beta \gamma$ dimer. Replacement of network residues prevents subunit dissociation, regardless of agonist or GTP binding. These findings reveal the molecular basis for the final committed step of $G$ protein activation.

\section{KEYWORDS}

G protein; molecular dynamics simulations; nuclear magnetic resonance spectroscopy; bioluminescence resonance energy transfer

\section{HIGHLIGHTS}

- Receptors promote GTP-GDP exchange and dissociation of G protein $\alpha$ and $\beta \gamma$ subunits

- We find an allosteric network linking the $\gamma$ phosphate of GTP with release of G $\beta \gamma$

- The network consists of a conserved Gly-Arg-Glu "activation triad"

- Triad mutations prevent subunit dissociation, regardless of agonist or GTP binding

- Triad mutations are responsible for human endocrine and neurological disorders 


\section{INTRODUCTION}

Many hormones, neurotransmitters, and clinically important drugs elicit their effects through $G$ protein coupled receptors (GPCRs). Receptors of this class represent one of the largest gene families, and by far the largest class of drug targets. Upon receptor activation by agonist, the $G$ protein $\alpha$ subunit releases GDP, binds GTP, and dissociates from the G $\beta \gamma$ dimer. The free $\mathrm{G} \alpha$ subunit then modulates the activity of downstream effector proteins such as adenylyl cyclase and phospholipase $\mathrm{C}$, among others. The $\mathrm{G} \beta \gamma$ dimer can regulate many of these same effectors as well as potassium channels, calcium channels, phosphatidylinositol-3 kinase and MAPKs. Signaling is terminated after G $\alpha$ hydrolyzes GTP and the heterotrimer reassembles. In most cases this inactivation step is accelerated by RGS proteins. Thus, receptors serve as signal discriminators while RGS proteins serve as timing devices and $G$ proteins serve as determinants of output specificity.

The effects of $G$ protein activation -- such as vasodilation, pain reduction, euphoria as well as light and odor sensing -- are quite diverse depending on the cellular context. Additional diversity comes from differential expression of $800+$ GPCRs, which includes members with little or no shared sequence similarity. In contrast, $G$ proteins are highly conserved in structure and function, and in mammals consist of only four subfamilies $\left(G_{s}, G_{i / o}, G_{q / 11}\right.$ and $\left.G_{12 / 13}\right)$ encoded by 16 individual genes.

The ability of such widely divergent receptors to activate a comparatively small number of $G$ proteins indicates a shared mechanism of activation (Flock et al., 2017; Flock et al., 2015). Broadly speaking, this process can be broken down into three discrete protein transformations: (i) ligand-specific conformational changes in the receptor, (ii) GTP-induced conformational changes in $G \alpha$, and (iii) displacement of the $G \beta \gamma$ subunits. It had long been postulated that an activated receptor must induce an opening of the two domains within $G \alpha$, stabilizing the nucleotide free state and facilitating GTP-GDP exchange. Direct evidence of such inter-domain opening was first provided through double electron-electron resonance experiments on rhodopsin-activated $\mathrm{G} \alpha_{i}$ (Van Eps et al., 2011) and on $\mathrm{G} \alpha_{s}$ (Dror et al., 2015), hydrogendeuterium-exchange studies (Chung et al., 2011) and through X-ray crystallographic studies of the $\beta_{2}$ adrenergic receptor bound to the $G_{s}$ protein heterotrimer (Rasmussen et al., 2011). Collectively, these findings indicate that $\mathrm{G} \alpha$ undergoes a "clam-shell like' opening to facilitate nucleotide release, and that both domains contribute to the process. In all of these structures however, $\mathrm{G} \beta \gamma$ remains associated with $\mathrm{G} \alpha$, even in the nucleotide-free and agonist-bound complex. Thus, the conformational changes associated with the release of nucleotide are necessary but not sufficient for the subsequent release of $G \beta \gamma$.

The third and final step of G protein activation begins with GTP binding. This entails conformational changes in three segments of $\mathrm{G} \alpha$ known as Switch I, II and III (Lambright et al., 1994). Crystal structures revealed that that Switch I connects the two domains of the $G \alpha$ subunit, comprised of a RAS-homologous domain and an all-helical domain. Switches II and III are located within the RAS-like domain and are disordered in the GDP-bound structure. Upon GTP binding Switch II forms a well-ordered helix and makes multiple contacts with residues in the $\alpha 3$ helix. Switches I and II interact directly with the $\gamma$-phosphate of GTP and form part of the G $\beta \gamma$-interacting surface (Lambright et al., 1996; Wall et al., 1995). Therefore, to form the active state, residues critical to maintaining $G \beta \gamma$ binding must retract inward toward the newly bound GTP molecule, creating a "molecular tug-of-war." Less is known about the mechanism behind the dynamic cascade of events linking the $\gamma$-phosphate to Switch I and II and the displacement of $\mathrm{G} \beta \gamma$. Here, by combining cross-species sequence analysis, Molecular Dynamics (MD) 
simulations, thermostability measurements, direct $G \alpha$ and $G \beta \gamma$ binding assays, NMR chemical shift analysis and cell-based functional assays we have uncovered the dynamic and transient effect of GTP binding leading to G $\beta \gamma$ dissociation. These models were also tested functionally, in biological contexts including the model organism S. cerevisiae. Our analysis reveals the existence of an evolutionarily conserved Gly-Arg-Glu triad that underpins all G protein activation. In particular, we show that these residues form an allosteric link between GTP and $\mathrm{G} \beta \gamma$. Mutations in any of these residues impair subunit dissociation, most likely due to a loss of a polar network between the terminal $(\gamma)$ phosphate of GTP and the G $\beta \gamma$-binding interface. Given that this motif is perfectly conserved among known $\mathrm{G} \alpha$ subunits, it is likely to be a universal feature of $G$ protein activity. Thus, our findings reveal how a single phosphate group controls $G$ protein subunit dissociation and signaling, processes that underlie much of human physiology and a substantial fraction of medical pharmacology. 


\section{RESULTS}

\section{Triad hypothesis}

The active state of a receptor is able to bind simultaneously to agonist and nucleotide-free $G$ protein; agonist binding stabilizes the empty form of the $G$ protein heterotrimer, thereby facilitating the exchange of GTP for GDP. Binding to either nucleotide favors release of G protein from receptor and of receptor from agonist (De Lean et al., 1980; DeVree et al., 2016). However, it is GTP alone that triggers the release of G $\beta \gamma$ from G $\alpha$ (Figure 1). Thus, the active state of the $G$ protein can be defined as the conformation stabilized by the terminal phosphate of GTP. Our goal here was to determine the molecular rearrangements responsible for this third and final committed step of $G$ protein activation.

To guide our experimental analysis, we began with a detailed examination of the known $\mathrm{G} \alpha$ structures. In particular we focused on the amino acids that bridge the $\gamma$-phosphate to the $G \beta \gamma$ binding domain. This analysis revealed a triad of amino acids (in $G \alpha_{i 1}$ numbering: $G 203$, R208, and E245) that are conserved in all G $\alpha$ proteins (Figure S1, Supplemental Information). On GTP binding, the glycine repositions the arginine and allows it to form a salt bridge with the glutamate. This Arg-Glu pair has been described previously as a molecular "hasp" or latch that fastens Switch II to the $\alpha 3$ helix and locks $G \alpha$ in the active conformation (Figure 1) (liri et al., 1997). Accordingly, the conserved glycine functions as a lock or pin that secures the latch and prevents access of $\mathrm{G} \beta \gamma$ to $\mathrm{G} \alpha$. In support of this model, small $\mathrm{G}$ proteins such as RAS contain the conserved glycine but lack the arginine or glutamate and do not bind to G $\beta \gamma$ subunits. Moreover, replacement of the conserved glycine in $G \alpha_{s}(G 226 A)$ or $G \alpha_{i 1}$ (G203A) disrupts formation of the Glu-Arg salt bridge (Berghuis et al., 1996), and appears to stabilize the protein in the $\mathrm{G} \beta \gamma$-bound state (Berghuis et al., 1996; Lee et al., 1992; Miller et al., 1988). Mutations in the arginine ( $\mathrm{G} \alpha_{s}-\mathrm{R} 231 \mathrm{H}$ and yeast Gpa1-R327S) likewise impede cell signaling by G $\beta \gamma$ (Apanovitch et al., 1998; liri et al., 1997). Based on our modeling and previous observations, we hypothesized that this triad of amino acids senses the presence of the $\gamma$ phosphate of GTP and allosterically controls subunit dissociation.

\section{Triad residues regulate $G \beta \gamma$ signaling in vivo.}

While RAS and $G \alpha$ are functionally related and share the same architecture, the acquisition of the arginine-glutamate latch may account for the ability of $\mathrm{G} \alpha$ to regulate $\mathrm{G} \beta \gamma$ release. As an initial test of our model, we replaced the conserved arginine and glutamate with all 19 other amino acids and tested their functionality in a systematic fashion. Given the large number of mutants and, given the need to isolate the effects of each mutant from other functionally similar $\mathrm{G} \alpha$ subtypes, we conducted our analysis in yeast. The yeast system has some unique advantages that would help in our interpretation of the data. Most notably, yeast express a single canonical GPCR and G protein, greatly simplifying analysis of structure-function relationships. This is in marked contrast to humans where multiple $G$ proteins receive signals from hundreds of distinct receptors. Moreover, $G$ proteins in yeast and animals have conserved structures, and heterologous expression studies indicate that the receptors and $\mathrm{G}$ protein subunits are functionally interchangeable (Dowell and Brown, 2009). Therefore, any perturbation in the yeast $\mathrm{G} \alpha$ are usually predictive of perturbations in mammalian $\mathrm{G}$ proteins.

We first compared pheromone signaling in cells that express wild-type or mutant forms of Gpa1, expressed using the native promoter in a single copy plasmid. All of our functional assays used the extensively-studied G323A mutant as a positive control. This substitution has been 
characterized mechanistically for $G \alpha_{s}$ and $G \alpha_{i 1}$, and demonstrated to reduce the binding affinity for magnesium and GTP (Berghuis et al., 1996; Lee et al., 1992; Miller et al., 1988). These mutants bind properly to receptors, GDP-P $\mathrm{P}_{\mathrm{i}}$ and $\mathrm{G} \beta \gamma$, and partially stimulate $(25-60 \%$ of full activity) adenylyl cyclase in vitro. Thus, the Gly-to-Ala mutants appear to be locked in a permanently $G \beta \gamma$-bound state, but are otherwise properly folded and competent to interact with other known binding partners. Based on these previous studies, and given the proximity of Gly323 to the guanine nucleotide, we predicted that G323A would exhibit the strongest suppression of $\mathrm{G}$ protein dissociation, making it our benchmark control.

We measured two outcomes of pheromone signaling. The first was $\mathrm{G} \beta \gamma$-mediated cell growth arrest (halo assay). Pheromone spotted onto a filter disk produces a zone of growth arrest, the size of which correlates with pheromone sensitivity. As shown in Figure 2, substitutions of Arg327 with Cys, Asn, Ser, and Val conferred the most effective inhibition (Figure 2A, bottom). Inhibition was absent when the arginine was replaced with glutamine. Other replacements yielded intermediate results. Most substitutions of the triad glutamate likewise yielded intermediate effects, with the strongest effect observed for E364K. None of the arginine or glutamate substitutions were as potent as the benchmark G323A mutant.

We then examined the effects of the triad mutations using a G $\beta \gamma$-dependent gene transcription assay. In this method, induction of a pheromone-inducible promoter (from FUS1) leads to increased expression of a reporter protein. As shown in Figure 2B, expression of Gpa1R327S caused a substantial reduction in reporter activity, but not to the extent of the benchmark G323A mutant. Thus, the two functional assays are largely in agreement and together show that pheromone signaling is diminished upon expression of some, but not all, triad mutants. Given that almost any substitution should disrupt the salt bridge that defines the hasp, we conclude that arginine and glutamate have unique functional properties that go beyond their ion-pair interaction.

\section{Triad residues control subunit dissociation.}

Our yeast-based measurements indicate that many, but not all, triad substitutions exhibit inhibitory effects on $\mathrm{G} \beta \gamma$ release. To determine if these functions are universally conserved, we introduced our best-performing mutations into human $\mathrm{G} \alpha$ subunits and tested their activity in a mammalian expression system. To this end we used an optimized protein proximity assay that detects receptor-stimulated release of $\mathrm{G} \beta \gamma$ from $\mathrm{G} \alpha$. In this bioluminescence resonance energy transfer (BRET) assay, a G $\alpha$-Renilla luciferase (RLuc) fusion is co-transfected with $\mathrm{G} \beta_{3}$ and a G $\gamma_{9}$-GFP fusion (Olsen et al., 2020). For these experiments we used the neurotensin receptor NTR1 because it couples efficiently to multiple $G \alpha$ subtypes including $G \alpha_{i}, G \alpha_{q}$ and $G \alpha_{s}$. When agonist stimulates the receptor, dissociation of the G $\alpha$-RLuc (energy donor) and G $\beta \gamma$-GFP (energy acceptor) is measured as the ratio of the emission of GFP to the emission of RLuc. As shown in Figure 3A, activation of all three $G$ proteins was substantially diminished by the triad substitutions, with the exception of the Arg-to-GIn replacement in $G \alpha_{i 3}$, (but not $G \alpha_{s}$ or $G \alpha_{q}$, discussed below). Signaling by the $\mu$-opioid receptor was likewise dampened by mutations in $\mathrm{G} \alpha_{i 3}$, indicating that the inhibitory effects are shared by multiple receptors and agonists (Figure S3, Supplemental Information). We conclude from these data that the triad residues are not only structurally conserved but are functionally conserved as well. These findings also demonstrate the utility of the yeast system for prioritizing mutants suitable for follow up analysis in a more complex but physiologically informative system. 
The data provided above indicate that the conserved triad is needed for proper G $\beta \gamma$ release in yeast and in human cells. To delineate the mechanism by which the triad acts, we tested the ability of the R208S mutant form of the protein to bind G $\beta \gamma$ directly. We focused on the conserved arginine because it connects the Switch II glycine (binds $\gamma$-phosphate) and the G $\beta \gamma$ binding interface. In addition, the R208S mutant exhibited the strongest inhibitory effect of the 19 substitutions tested. For this analysis we chose bio-layer interferometry (BLI), which analyzes the interference pattern of white light reflected from two surfaces: a layer of immobilized protein on the biosensor tip, and an internal reference layer. We combined biotin$\mathrm{G} \beta_{1} \gamma_{2}$, immobilized onto Streptavidin-coated biosensor tips, with a solution containing either 1 $\mu \mathrm{M}$ or $3 \mu \mathrm{M}$ purified $\mathrm{G} \alpha_{i 1}$ or $\mathrm{G} \alpha_{\mathrm{i1}}-\mathrm{R} 208 \mathrm{~S}$, and equilibrated the protein in either GDP or GDP plus aluminum fluoride $\left(\mathrm{AlF}_{4}{ }^{-}\right.$). Aluminum fluoride mimics the pentavalent transition state for GTP hydrolysis (Mixon et al., 1995; Sondek et al., 1994). Thus, the structure of the $\mathrm{AlF}_{4}{ }^{-}$state is very similar to the active GTP-bound state and preserves the triad polar network, but activation by $\mathrm{AlF}_{4}-$ does not depend on nucleotide exchange. After 5 min the tips were transferred to an identical solution but lacking $\mathrm{G} \alpha_{\mathrm{i} 1}$. By this measure, the wildtype (Figure 3B, left) and R208S mutant (Figure 3B, right) exhibited similar association and dissociation kinetics in the presence of GDP (red and pink traces) (Table S1, Supplemental Information). As expected, GDP-AlF $_{4}-\mathrm{G} \alpha_{\mathrm{i} 1}$ bound more poorly to $\mathrm{G} \beta \gamma$ (black and grey traces), with a slower initial on rate and lower maximum binding at both concentrations of $\mathrm{G} \alpha$ subunit. In contrast, and in support of our model, G $\beta \gamma$ bound equally well to the "active" and "inactive" forms of the R208S mutant. The lack of an $\mathrm{AlF}_{4}{ }^{-}$-dependent alteration of the R208S binding to $\mathrm{G} \beta \gamma$ is consistent with the BRET data obtained in HEK293 cells (Figure 3A). Thus, the conserved arginine mediates the assembly of the triad and formation of an active state conformation leading to a lower affinity of $\mathrm{G} \alpha$ for $\mathrm{G} \beta \gamma$.

\section{A dynamic mechanism for the $\gamma$-phosphate of GTP in coordinating the active-state conformation of $\mathbf{G} \alpha$.}

To provide a dynamic view of events leading to dissociation of the G $\beta \gamma$ subunits, we performed all-atom MD simulations of $\mathrm{G} \alpha_{\mathrm{i1}}$ in complex with $\mathrm{G} \beta \gamma$ and bound to either GDP or GTP $\gamma \mathrm{S}$. We also simulated the dynamics of $\mathrm{G} \alpha_{i 1}-\mathrm{R} 208 \mathrm{~S}, \mathrm{G} \alpha_{i 1}-\mathrm{E} 245 \mathrm{~K}$ and $\mathrm{G} \alpha_{i 1}-\mathrm{G} 203 \mathrm{~A}$, all bound to GTP $\gamma$ S. Finally, as a counter example to these loss-of-signaling mutants, we included in our analysis $\mathrm{G} \alpha_{\mathrm{i1}}-\mathrm{R} 208 \mathrm{Q}$, which is unique in its ability to retain partial activity. Our analysis of the crystal structures of the GDP-bound trimeric $G \alpha_{i 1} G \beta \gamma$ (Wall et al., 1995) compared to GTP $\gamma$ S-bound $\mathrm{G} \alpha_{i 1}$ (Coleman et al., 1994) showed that the residues in Switch II and Switch III form tighter interactions when bound to GTP $\gamma$ S and enable G $\beta \gamma$ dissociation. To assess how mutants affect the strength of subunit interaction, we calculated the non-bond interaction energies between the $\mathrm{G} \alpha$ and the $\mathrm{G} \beta$ subunits, averaged over MD trajectories for each of the mutant and wild type proteins. We also calculated the interaction energies between the residues in the Switch II and the Switch III regions (see Methods section for more details). As anticipated, the G $\alpha-G \beta$ subunit interaction energies varied inversely with the interaction energy between the Switch II and Switch III regions, shown in the plot in Figure 3C. In contrast, the non-signaling $G \alpha_{i 1}$ mutants showed $G \alpha-G \beta$ interaction energies similar to that of GDP-bound wildtype $G \alpha_{i 1}$ even when bound to GTP $\gamma$ S.

To understand the mechanism of this dynamic "pull-push" effect we analyzed the residue interaction network emerging from the $\gamma$-phosphate of GTP connecting to Switch I, and cascading further to Switch III and the Switch II region, which forms the G $\beta$ interface. As shown in the network diagram in Figure 3D, we identified an extensive polar network linking the $\gamma$ - 
phosphate group to Arg178 in Switch I. This pull breaks the interaction of Arg178 with Glu43 in the P-loop, which in turn strengthens the interaction of Glu43 with Arg242 in the Switch III region. This interaction between Glu43 and Switch III leads to the triad formation, comprised of Glu245-Arg208 and Gly203 in the Switch II region. Additional polar interactions between Arg242, Asp237, Glu236 in Switch III with Arg205 in Switch II also strengthen the Switch II Switch III interactions. This Switch III - Switch II pulling effect in turn weakens the polar network of interactions of Lys210 and Lys209 in Switch II with Asp246, Asn230 and Asp228 residues in the $\mathrm{G} \beta$ subunit. Breaking $\gamma$-phosphate interaction with Arg178 in the GDP-bound G $\alpha_{i 1}$ disrupts the cascade of polar interactions between Switch I, Switch III and Switch II, thereby breaking the triad and preserving the polar interaction network between Switch II and the G $\beta$ interface as shown in Figure 3D. A similar interaction network mechanism was also observed for $G \alpha_{s}$ MD simulations (Figure S3, Supplemental Information). These results reveal a common mechanism leading to $G \beta \gamma$ dissociation. Specifically, we have identified an allosteric link between the terminal phosphate of GTP and the G $\alpha-G \beta \gamma$ interface.

\section{Triad mutants confer a GDP-like conformation on GTP-bound $G \alpha_{i 1}$}

Our cell-based assays indicate that mutations in the triad arginine and glutamate confer sustained binding to $G \beta \gamma$. The experimental BRET (Figure 3A) agrees reasonably well with the subunit interface interaction energy calculated from MD simulations. Our MD simulations suggested that these mutants lack important conformational changes associated with GTP binding, specifically changes in Switch II and Switch III, and that these structural differences are propagated by the $\gamma$-phosphate of GTP. To that end, we monitored the conformational stability of all three mutants when bound to guanine nucleotides.

We have previously shown that $G \alpha_{i 1}$ is thermodynamically more stable when bound to GTP $\gamma$ than to GDP (Isom et al., 2013). This difference is consistent with structural studies showing that the GTP-bound state is considerably more rigid than the GDP-bound state, as dictated by regions near the $\gamma$-phosphate group and at the G $\beta \gamma$ interface (Sun et al., 2015). Since the triad mutants function as if they were bound to GDP, we anticipated that they would exhibit a reduced thermostability even if bound to GTP. To test this, we purified wildtype and several mutant forms of $G \alpha_{i 1}$ and determined the melting temperature of each using the quantitative cysteine reactivity (fQCR) assay. In this method, cysteine residues are labeled with a fluorogenic reagent by a mechanism that is analogous to hydrogen exchange. Upon heating, protected cysteine residues of $\mathrm{G} \alpha$ become exposed and can be covalently labeled by cysteine-specific probes (Isom et al., 2013). Using a two-state model of denaturation, we analyzed each $\mathrm{G} \alpha_{\mathrm{i} 1}$ mutant unfolding profile to quantify the midpoint of temperature unfolding $\left(T_{\mathrm{m}}\right)$. Representative thermostability profiles for the wildtype and triad mutant forms of $G \alpha_{i 1}$ are shown in Figure $4 A$. These experiments confirm that the wildtype protein undergoes a $9{ }^{\circ} \mathrm{C}$ increase in $T_{\mathrm{m}}$ when bound to GTP $\gamma$ S in place of GDP; in contrast, the mutants exhibited little or no change. We conclude that that the conserved triad confers critical conformational changes that favor the dissociated state of the $G$ protein.

Structural dynamics studies have shown that thermostable mutants exhibit conformational homogeneity, meaning fewer distinct conformations in the ensemble during dynamics simulations (Vaidehi et al., 2016). The conformational homogeneity manifests as fewer energy minima in the free energy surface of thermostable mutants (Ghosh et al., 2018). To understand the structural basis for the observed thermostability differences, we generated the free energy surface of GDP- and GTP $\gamma$ S-bound $\mathrm{G}_{i 1}$ using MD simulation trajectories. Figure 4B shows the free energy surface of $G \alpha_{i 1}$ bound to GTP $\gamma$ S and GDP, along with the $G \alpha_{i 1}$ mutants bound to 
GTP $\gamma$ S. In particular, we observed that $\alpha_{i_{1}-G T P}$ S had fewer energy minima and was less conformationally homogeneous compared to the mutant forms of $G \alpha_{i 1}$. Thus, there is a correlation between conformational heterogeneity and thermal instability.

A comparison of crystal structures of GDP- and GTP $\gamma \mathrm{S}$-bound $\mathrm{G} \alpha_{\mathrm{i} 1}$ shows that residues in Switch II fold into a helix leading to the dissociation of $G \beta \gamma$. We speculated that formation of an ordered helical region in Switch II in GTP $\gamma$ S-bound G $\alpha_{i 1}$ could lead to its enhanced thermostability. Therefore, we calculated the average helicity of the residues in the Switch II region averaged over all the MD snapshots in the most occupied conformation cluster extracted from the free energy surfaces for the wildtype and mutant forms of $\mathrm{G \alpha}_{\mathrm{i1}}$. As shown in Figure $4 \mathrm{C}$, Switch II transitioned from about 15\% to 63\% helicity in going from GDP-bound to GTP $\gamma$ S-bound $\mathrm{G} \alpha_{\mathrm{i1}}$. It is important to note that we started the MD simulations from the same disordered conformation in Switch II observed in the crystal structure of the GDP-bound G $\alpha_{i 1}$ trimeric structure. MD simulations recapitulate the loop-to-helical transitions that are triggered by the $\gamma$ phosphate group of the GTP $\gamma$ S engaging the dynamic mechanism shown in Figure 3D. To examine if the loop-to-helix transitions lead to increased thermostability, we calculated the average interaction energies between the $\mathrm{G} \alpha$ and $\mathrm{G} \beta$ subunits averaged over the MD snapshots in the most occupied conformation cluster, extracted from free energy surfaces. The calculated helicity of Switch II correlates inversely with the $G \beta \gamma$ interaction energies for wildtype $\mathrm{G} \alpha_{i 1}$, bound to GDP and GTP $\gamma \mathrm{S}$, as well as the G $\alpha_{i 1}$ mutants bound to GTP $\gamma \mathrm{S}$ (Figure 4C). In summary, these findings indicate that increased helicity could bring a more ordered structure to the Switch II region and confer thermostability and conformational homogeneity for $G \alpha_{i 1}$ bound to GTP $\gamma$ S.

An alternative interpretation is that the mutants bind to GDP but not GTP. To test this experimentally we determined the rate of nucleotide exchange, measured as a gain of fluorescence as BODIPY-GTP $\gamma$ S bound to protein (McEwen et al., 2001). As shown in Figure $4 D$, the labeled analog bound to mutants as well (or faster) than the wildtype protein. We then added an excess of unlabeled GTP $\gamma \mathrm{S}$ and determined that the rate of dissociation was substantially faster for all of the mutants as compared to that of wildtype. We infer that the triad mutants confer a more dynamic binding pocket, which results in faster dissociation of nucleotide. More broadly, these data indicate that loss of the triad residues does not impede GTP binding or dissociation, but prevents the conformational changes required for $\mathrm{G} \alpha$ activation and $\mathrm{G} \beta \gamma$ dissociation. Taken together, our MD simulations and biophysical assays confirm that mutations in the triad arginine and glutamate place the protein in a permanently "inactive" conformation, compatible with sustained binding to G $\beta \gamma$.

The results presented above reveal the unique importance of the triad arginine. Generally speaking, substitutions at this site lead to diminished signaling by $G \beta \gamma$. However, as detailed above (Figures 3 and 4), substitution of the arginine with glutamine partially preserves function, but only in $\mathrm{G} \alpha_{\mathrm{i} 1}$ and not in $\mathrm{G} \alpha_{\mathrm{s}}$. In accordance with these observations, our calculations of interaction energies (Figure 4C), thermal stability measurements (Figure 4E), computed free energy surfaces (Figure 4F), and helicity calculations (Figures $4 \mathrm{C}$ and $\mathrm{G}$ ) revealed that the R208Q mutant is more similar to wildtype than any of the other arginine mutants. The weakening of interaction between $G \alpha_{i 1}$ and the $G \beta \gamma$ subunits of $G$ protein is caused by the enhanced interaction between the Switch II and Switch III regions within the $G \alpha_{i 1}$ subunit. These simulations are the theoretical basis for the proposed competition between G $\beta \gamma$ and Switch III for Switch II (Figure 4H). The calculated interaction energies for $\mathrm{G} \alpha$ and $\mathrm{G} \beta \gamma$ subunits become less favorable as the interaction energies for Switch II and Switch III become more favorable, 
and vice versa (primary and secondary y-axis respectively, Figure $3 \mathrm{C}$ ). The same paradigm holds for $\mathrm{G} \alpha_{s}$ (Figure 4I). Thus, our molecular dynamics simulations were able to predict substitution- and subtype-specific dissociation events with exquisite accuracy and mechanistic detail.

We then sought to determine the structural basis for the unique ability of $G \alpha_{i 1}-R 208 Q$ to sustain signaling. To that end, we compared the structure of wild type $G \alpha_{i 1}$ with a new X-ray crystal structure of $\mathrm{G} \alpha_{i 1}-\mathrm{R} 208 \mathrm{Q}$, both in the GTP $\gamma \mathrm{S}$-bound state (Figure 4J, compare left and right panels). This analysis revealed that $G \ln 208$, like the native arginine of $G \alpha_{i 1}$, is close enough to form a $\mathrm{H}$-bond network with the conserved glycine and glutamate. In contrast, and as anticipated by our MD simulations, the same substitution would not restore the network in $G \alpha_{s}$ or $\mathrm{G} \alpha_{\mathrm{q}}$. Whereas Arg208 of $\mathrm{G} \alpha_{\mathrm{i} 1}$ faces Switch III in both the active and inactive states, the corresponding residue in $\mathrm{G} \alpha_{s}$ must rotate $\sim 180^{\circ}$ before it can interact with the conserved glycine and glutamate.

Our simulations appear to account for the distinct functional properties of the G-R-E motif, as determined by thermostability measurements, nucleotide and protein binding measurements, as well as the signaling outputs obtained in yeast and animal cells. Most strikingly, the atomistic MD simulations anticipated the unique and unexpected differences between $G_{i}$ and $G_{s}$, and the differences between R208Q and other Arg208 substitutions. The differences between $\mathrm{G} \alpha_{i 1}$ R208Q and $G \alpha_{s}-R 231 Q$ in particular support our model that the arginine has functions beyond simply forming a salt bridge with the conserved glutamate. More broadly, these results highlight the predictive power of MD simulations, particularly when integrated with molecular and cellular experimental analysis.

\section{The triad arginine controls the final committed step of $\mathbf{G}$ protein activation.}

Finally, we sought to determine the structural basis for the unique functional properties of the R208S mutation. Based on the mechanism from MD simulations, we postulated that the mutant retains $\mathrm{G} \beta \gamma$ binding by disrupting the conserved polar network that dictates the response to GTP. To test this directly, we collected ${ }^{1} \mathrm{H}-{ }^{15} \mathrm{~N}$ 2D heteronuclear NMR spectra of wild-type and mutant $\mathrm{G}_{i 1}$, both in the presence of GDP and GTP $\gamma \mathrm{S}$. This method allows for the detection of backbone and side-chain $\mathrm{NH}$ resonances. As an $\mathrm{NH}$ resonance can be detected for every residue with the exception of proline, the spectrum contains a "fingerprint" of the protein backbone and perturbations resulting from changes in intramolecular interactions. We consider this a definitive method for detecting the conformational changes that accompany nucleotide binding and $G$ protein activation. In contrast to some other $\mathrm{G} \alpha$ isoforms and most members of the RAS family of GTPases, $G \alpha_{i 1}$ undergoes nucleotide exchange within minutes, even in the absence of exchange factor or receptor (Figure 4D).

As shown previously (Goricanec et al., 2016), a substantial number of Switch II peaks (Figure 5 arrows) for wildtype $\mathrm{G} \alpha_{i 1}$ were resolved in the presence of GTP $\gamma \mathrm{S}$ (black) but not GDP (blue). These data are consistent with X-ray structural data, indicating that Switch II is more ordered in the activated state as compared with the inactive state. Likewise, $\mathrm{G} \alpha_{i_{1}}-\mathrm{R} 208 \mathrm{~S}$ exhibited multiple peak shifts when GDP was replaced with GTP $\gamma$ S, indicating that nucleotide exchange had occurred (Figure S5 top, Supplemental Information). Consistent with our fast exchange data for R208S, the mutant GTP $\gamma$ S spectrum contains a shifted tryptophan that indicates GTP $\gamma$ S binding

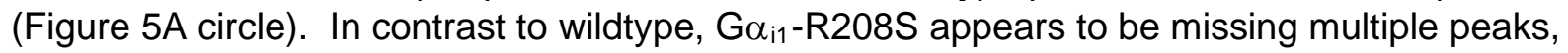
consistent with a more dynamic GTP $\gamma$ S state. In support of our observations from MD 
simulations (Figure S3 Supplemental Information), many resonances missing in the mutant spectrum were in the Switch regions for wildtype bound to GTP $\gamma \mathrm{S}$ (Figure 5B). These results indicate that $\mathrm{G} \alpha_{\mathrm{i} 1}-\mathrm{R} 208 \mathrm{~S}$ is in an alternate, more dynamic conformational ensemble, even when bound to GTP $\gamma$ S. More broadly, these results indicate that the conserved arginine is necessary to detect the presence of the $\gamma$-phosphate and undergo the structural changes necessary to maintain the active state. 


\section{DISCUSSION}

Heterotrimeric $\mathrm{G}$ proteins are present in a wide variety of organisms and exist in multiple distinct subclasses. Recent findings, including structure determinations of receptor-bound G proteins, have revealed the molecular motions leading to the release of GDP. Here we considered the subsequent molecular events leading to the release of G $\beta \gamma$. Our approach combined crossspecies sequence analysis, cell-based functional assays, MD simulations, thermostability measurements, direct $\mathrm{G} \alpha$ and $\mathrm{G} \beta \gamma$ binding measurements, and NMR spectroscopy.

Our study revealed the existence of a network of highly conserved amino acids ("G-R-E motif") that dictates the release of $\mathrm{G} \beta \gamma$ by $\mathrm{G} \alpha$. In the presence of the terminal phosphate of GTP, the conserved glycine forms a polar network with the arginine and glutamate, disrupting the $G \alpha-G \beta \gamma$ binding interface. By systematically replacing triad residues, and measuring the functional consequences of those changes, we demonstrated its unique importance in G protein activation in cells. Whereas a single phosphate can initiate subunit dissociation, we show that mutation of a single amino acid can override the process entirely. We then determined the molecular basis of activity through biochemical and biophysical experiments conducted in vitro. Finally, we established the atomic interactions responsible for dissociation of the $G$ protein subunits in silico.

Our findings provide the molecular basis for important functional behaviors documented through cellular genetic studies conducted almost 40 years ago. The $\$ 49$ cyc $^{-}$cell line, which bears the $\mathrm{G} \alpha_{\mathrm{s}}-\mathrm{G} 226 \mathrm{~A}$ mutation and is deficient in cAMP production (Bourne et al., 1981; Salomon and Bourne, 1981), was instrumental in the discovery of G proteins (Gilman, 1987). In yeast, spontaneous mutations at the triad glycine, glutamate and arginine confer strong resistance to pheromone stimulation (Apanovitch et al., 1998; Stratton et al., 1996). Replacement of the arginine was even sufficient to block unrestrained signaling in the complete absence of GTP hydrolysis (Apanovitch et al., 1998). The importance of the triad arginine has also been revealed through human genetic studies. The conserved arginine is one of two sites in $\mathrm{G} \alpha_{\mathrm{s}}$ mutated in patients with pseudohypoparathyroidism 1a, a syndrome characterized by Albright hereditary osteodystrophy and resistance to hormone stimulation (Farfel et al., 1996; liri et al., 1997). More recent studies indicate that the triad arginine and glutamate are mutation "hotspots" in $\mathrm{G} \alpha_{0}$, frequently altered in individuals with neurodevelopmental disorders including involuntary movement and seizures. Of more than 30 missense or codon deletion mutations identified so far, at least six affect the conserved arginine or glutamate (https://monarchinitiative.org/gene/HGNC:4389\#overview). All of the phenotypes associated with loss of the G-R-E motif, in yeast and humans, can be attributed to sustained subunit association.

Finally, our analysis of $\mathrm{G}$ protein activation complements recent advances in our understanding of receptor activation. The first crystal structure of a receptor-G protein complex, published in 2011 (Rasmussen et al., 2011), provided detailed insights into the conformational changes leading to nucleotide release. Most notably, that structure revealed a dramatic displacement of the $\mathrm{G} \alpha$ helical domain from the RAS-like domain, thereby exposing the nucleotide binding site. A major impediment to obtaining such structures is the difficulty of assembling an agonist-bound receptor with a nucleotide-free $G$ protein. The first structures used fusions and single chain antibodies to stabilize this otherwise ephemeral state. Subsequent studies used variants of $G \alpha$ containing a panel of up to 8 mutations, including substitutions of the triad glycine and glutamate (Draper-Joyce et al., 2018; Liang et al., 2018a; Liang et al., 2018b). Based on our findings, 
substitutions of the G-R-E motif are likely to be particularly useful in obtaining stabilized heterotrimer complexes suitable for structural analysis in the future.

Taken together, our analysis reveals events leading to the final committed step of $G$ protein activation and provides a mechanistic basis for human disease. The structural rearrangements leading to subunit dissociation are more subtle than those affecting nucleotide exchange, but they are just as consequential. In both cases, conserved residues form a network of noncovalent contacts that link a bound ligand - either agonist or GTP - to a network of contacts needed to release $\mathrm{G} \beta \gamma$. Given that the G-R-E triad residues are conserved in all $\mathrm{G}$ proteins, the allosteric mechanism detailed here is likely to be universal for all subtypes and species. Just as a detailed understanding of the molecular basis for ligand binding has led to important advances in pharmacology, a better understanding of subunit dissociation could reveal strategies to bypass or compensate for the human genetic defects that cause disease. 


\section{ACKNOWLEDGEMENTS}

Funded by NIH grants F31NS093917 (R.H.J.O.), R35-GM134962 (S.L.C.), R35-GM127303 (A.V.S.), R01-GM117923 (N.V.), R35-GM118105 (H.G.D).

\section{AUTHOR CONTRIBUTIONS}

Authors S.L.C., T.L., R.H.J.O., A.V.S., N.H.V., and G.Y. are listed alphabetically

K.M.K., S.G., N.V., H.G.D.: Conceptualization

K.M.K., S.G., S.L.C., T.L., R.H.J.O., A.V.S., N.H.V., G.Y., N.V., H.G.D.: Formal Analysis

S.L.C., A.V.S., N.V., and H.G.D.: Funding acquisition

K.M.K., S.G., T.L., N.H.V., G.Y.: Investigation

S.L.C., A.V.S., N.V., H.G.D.: Project administration

S.L.C., R.H.J.O., A.V.S., N.V., H.G.D.: Supervision

K.M.K., S.G., S.L.C., T.L., R.H.J.O., A.V.S., N.H.V., G.Y., N.V., and H.G.D.: Validation

K.M.K., S.G., N.V., H.G.D.: Writing - original draft

K.M.K., S.G., S.L.C., T.L., R.H.J.O., A.V.S., N.H.V., G.Y., N.V., H.G.D.: Writing - review \& editing

\section{DECLARATION OF INTERESTS}

The authors declare no competing interests. 


\section{FIGURE LEGENDS}

Figure 1. Schematic of the $\mathrm{G}$ protein triad hypothesis.

Top, schematic representation of the G protein (grey) bound to GDP (left), none (center), and GTP (right). Cylinders represent the movements of Switch II and Switch III.

Bottom, overlaid structural representation of $\mathrm{G} \alpha_{\mathrm{i} 1}$ and $\mathrm{G} \alpha_{\mathrm{s}}$ (grey) bound to GDP (PDB: 1GP2 6CRK) (Maeda et al., 2018; Wall et al., 1995), none (apo state, PDB:6DDE 3SN6) (Koehl et al., 2018; Rasmussen et al., 2011), and GTP (PDB:1GIA and 1AZT) (Coleman et al., 1994;

Sunahara et al., 1997). The overlaid residues for the conserved triad are highlighted in black.

Figure 2. Triad residues regulate $\mathbf{G} \beta \gamma$ signaling in vivo. Wild-type yeast cells transformed with a single copy plasmid containing GPA1 or the indicated mutant G323A (GA), R327S (RS) or E364K (EK).

Left, plate assay with filter disks containing 75,25 , or $8 \mu \mathrm{g} \alpha$-factor pheromone.

Right, transcription reporter assay after treatment with the indicated concentration of $\alpha$ factor pheromone.

Bottom, quantitation of mutant phenotypes. Values indicate extent of growth inhibition, ranging from least (-) to most (+++) turbidity.

Figure 3. Triad residues control subunit dissociation.

(A) HEK293 cells transfected with the indicated wildtype or mutant $G \alpha-R L u c 8$ donor and $G \gamma-$ GFP acceptor proteins. Concentration-response measurements $(n=2)$ using the neurotensin receptor are presented as fold-decrease in dynamic range (dissociation, Net BRET).

(B) Purified biotinylated-G $\beta$ and $G \gamma$ immobilized on streptavidin exposed for $300 \mathrm{sec}$ (bar) to the indicated concentration of purified $\mathrm{G} \alpha_{i 1}$ (WT, left) or $\mathrm{G} \alpha_{i 1}-\mathrm{R} 208 \mathrm{~S}$ (RS, right), equilibrated in either GDP (red or pink) or GDP and aluminum fluoride (AlF ${ }_{4}^{-}$, black or grey). Binding is reported as a shift in the interference pattern $(\mathrm{nm})$.

(C) Plot of interaction energies between $G \alpha$ and $G \beta$ subunits versus interaction energies between residues in Switch II and Switch III regions in wildtype and the indicated G $\alpha_{\mathrm{i} 1}$ mutants. The interaction energies are averaged over the snapshots of the MD simulation trajectories. (D) A dynamic mechanism for the effect of the $\gamma$-phosphate group triggering the release of the $\mathrm{G} \beta \gamma$ subunits. The dynamic pull-push effect from the $\gamma$-phosphate group of GTP $\gamma \mathrm{S}$, that forms the polar residue interaction network involving the P-loop, Switch I, Switch II and Switch III residues, derived from MD simulations for wildtype $G \alpha_{i 1}$ bound to GTP $\gamma$ S (top) or GDP (bottom). The nucleotides are shown in ball and stick representation. The blue and the red dotted lines indicate sustained and broken residue contacts, respectively. Right panels show residues involved in the polar network for the GTP $\gamma \mathrm{S}$ - and GDP-bound $\mathrm{G}_{{ }_{i 1}}$ switch regions (black and gray colors respectively).

Figure 4. Triad residues coordinate the active-state conformation.

(A) Thermostability of purified wildtype and the indicated $G \alpha_{i 1}$ mutants equilibrated in either GTP $\gamma \mathrm{S}$ (solid lines) or GDP (dashed lines). $T_{\mathrm{m}}$ values were quantified by fitting a two-state model of thermal unfolding $(n=3)$.

(B) Free energy surfaces computed using population densities show the conformational heterogeneity of $G \alpha_{i 1}$ and its mutant systems. The projections of the surfaces are shown on the principal components 1 and 2 (PC1 and PC2) axes. The various conformational clusters are numbered (magenta) ordered by the population of each conformation cluster. Cluster 1 for example, is the most populated conformation cluster. The $Z$ axis is the Gibb's free energy obtained by inverting the population of these microstates and is shown as a colored heatmap. (C) Helicity of residues in the Switch II regions of wild type and the indicated $G \alpha_{i 1}$ mutants, 
bound to GTP $\gamma$ S, calculated as an average over the snapshots in the most occupied conformational cluster I from the free energy surfaces.

(D) Association of $6 \mu \mathrm{M}$ purified wildtype and the indicated $\mathrm{G} \alpha_{\mathrm{i1}}$ mutants with $100 \mathrm{nM}$ BODIPYGTP $\gamma$ S, followed after $600 \mathrm{~s}$ by addition of $50 \mu \mathrm{M}$ unlabeled GTP $\gamma \mathrm{S}(\mathrm{n}=2)$.

(E) Thermostability of purified wildtype $G \alpha_{i 1}$ and R208S mutant, as detailed above (A).

(F) Free energy surfaces computed using population densities calculated for principal components PC1 and PC2 for G $\alpha_{i 1}-\mathrm{R} 208 \mathrm{~S}$, as detailed above (B).

(G) Structural representation of the Switch II regions of $\mathrm{G} \alpha_{i 1}-\mathrm{R} 208 \mathrm{~S}$ bound to GTP $\gamma \mathrm{S}$, as detailed above $(\mathrm{C})$.

(H) A dynamic mechanism for the effect of the $\gamma$-phosphate group triggering the release of the $\mathrm{G} \beta \gamma$ subunits for $\mathrm{G} \alpha_{i 1}-\mathrm{R} 208 \mathrm{Q}$ as detailed above in Figure 3D.

(I) A dynamic mechanism for the effect of the $\gamma$-phosphate group triggering the release of the $\mathrm{G} \beta \gamma$ subunits for $\mathrm{G} \alpha_{\mathrm{s}}-\mathrm{R} 231 \mathrm{Q}$ as detailed above in Figure 3D.

(J) Crystal model of $G \alpha_{i 1}$ and $G \alpha_{i 1}-R 208 Q$ triad residues and GTP $\gamma S$ (ball and stick) from PDB: $1 \mathrm{GIA}$ and $6 \mathrm{M} 8 \mathrm{H}$.

Figure 5. The triad arginine controls the final committed step of $G$ protein activation. $2 \mathrm{D}\left[{ }^{15} \mathrm{~N},{ }^{1} \mathrm{H}\right]-\mathrm{HSQC}$ NMR of $\mathrm{G} \alpha_{\mathrm{i} 1} \Delta 31$ wildtype and R208S mutant.

(A) Overlay of wildtype $G \alpha_{i 1} \Delta 31$ in complex with GDP (Blue) and R208S G $\alpha_{i 1}$ in complex with GTP $\gamma$ S (Red).

(B) Overlay of wildtype (Black) and R208S (Red) G $\alpha_{i 1}$, each in complex with GTP $\gamma$ S (Black). Switch II specific peaks are labeled (arrows). 


\section{STAR METHODS}

\section{Resource Availability.}

Lead Contact: H.G.D.

Materials Availability: The study did not generate new unique reagents

Data and Code Availability: Data are available from H.G.D. and N.V. The study did not generate new unique code. NMR data will be made available at: http://www.bmrb.wisc.edu

Mutagenesis. Primestar Max (Takara Bio) mutagenesis was performed using primers containing 5 bp 5' to the codon and 20-30 bp 3' to the codon (Table S2, Supplemental Information).

Halos. Pheromone-induced growth inhibition was measured as described previously (Hoffman et al., 2002). Briefly, 75,25 , or $8 \mu \mathrm{g}$ synthetic $\alpha$ factor was spotted onto paper disks. Agar (0.5\%) was melted and then kept at $55^{\circ} \mathrm{C}$ before mixing with $100 \mu \mathrm{L}$ saturated culture of $S$. cerevisiae strain BY4741 bar1::KanMX (bar1 $\Delta)$, transformed with pRS316 containing either no insert (vector), GPA1 or mutant GPA1 (Song et al., 1996), and grown at $30^{\circ} \mathrm{C}$ in selective medium. After spreading the agar/yeast mixture, each disk was applied to one of three predefined locations on the surface of the agar. Cell growth was imaged after 24 and $48 \mathrm{hr}$.

Transcription. Pheromone-induced gene induction was monitored as described previously (Shellhammer et al., 2019). Briefly, BY4741 bar1s cells cotransformed with plasmids pRS426pFUS1-YeGFP3 (Shellhammer et al., 2019) and pRS316-GPA1 (wildtype or mutant) were grown to saturation then diluted to $\mathrm{OD}_{600}<0.001$. After reaching $\mathrm{OD}_{600}$ of 0.8 , synthetic $\alpha$ factor was added at defined concentrations. After $1.5 \mathrm{hr}$, GFP fluorescence was measured using a Molecular Devices Spectramax i3x plate reader at an excitation wavelength of $483 \mathrm{~nm}$ and emission wavelength of $518 \mathrm{~nm}$.

Bioluminescence Resonance Energy Transfer (BRET) assay. HEK293T cells (ATCC, \#CRL11268) were used to measure basal association and receptor-mediated dissociation of $G \alpha$ and $\mathrm{G} \beta \gamma$, as described previously (Olsen et al., 2020). Briefly, cells were transfected with plasmids encoding the neurotensin or $\mu$-opioid receptor, $\mathrm{G} \alpha$ fused to Renilla luciferase 8 (Rluc8), G $\beta_{3}$ and $\gamma_{9}$ - fused to GFP in a 1:1:1:1 ratio (cell density 750,000 cells in $3 \mathrm{~mL}$ ). After transfection (approximately $16 \mathrm{hr}$ ) cells were plated into poly-D-lysine coated 96-well white clear bottom plates in medium containing Dulbecco's Modified Eagle Medium $+1 \%$ dialyzed fetal bovine serum and then incubated overnight. The following day, cells were washed twice with assay buffer (20 mM (4-(2-hydroxyethyl)-1-piperazineethanesulfonic acid) (HEPES), Hank's Balanced Salt Solution, pH 7.4). $10 \mu \mathrm{L}$ of Rluc8 substrate (coelentrazine 400a, Nanolight) was added per well at a final concentration of $5 \mu \mathrm{M}$. Cells were then incubated 5 min in the dark. $30 \mu \mathrm{L}(3 \mathrm{X})$ of agonist in drug buffer (contains assay buffer, $0.1 \%$ bovine serum albumin) was added per well then incubated for $5 \mathrm{~min}$ in the dark. Plates were read for luminescence at 485 $\mathrm{nm}$ and fluorescence emission at $530 \mathrm{~nm}$ for $1 \mathrm{sec}$ per well using a Mithras LB940 multimode microplate reader. The BRET ratio was determined by dividing fluorescence by luminescence (GFP/Rluc). The receptor-catalyzed dissociation of the heterotrimer (net BRET) was measured by comparing the energy transfer from donor to acceptor and reported as ratios: GFP/Rluc per well - Basal BRET (GFP/Rluc at lowest dose of agonist) = Net BRET/ dissociation. The net BRET was plotted as the GFP/Rluc ratio as a function of neurotensin and the curve was fit in Graphpad Prism 8 (Graphpad Software Inc., San Diego, CA) (Che et al., 2020). 
Expression and Purification of $\mathbf{G} \alpha_{\mathrm{i1}}$. RIPL (Agilent) or Rosetta (Novagen) cells were transformed with PET-SUMO-G $\alpha_{i 1}$ plasmid (Maly and Crowhurst, 2012) and grown to saturation in $\mathrm{YZ}$ medium containing $0.02 \%(\mathrm{w} / \mathrm{v})$ glucose and $0.2 \%(\mathrm{w} / \mathrm{v})$ ala-lactose; autoinduction occurred via inhibition of the Lac repressor when ala-lactose becomes the primary fuel source (Isom et al., 2013). The culture temperature was lowered from 37 to $18{ }^{\circ} \mathrm{C}$ and allowed to rotate overnight. The cultures were harvested by centrifugation for $1 \mathrm{hr}$ at $4{ }^{\circ} \mathrm{C}$ and then lysed by sonication on ice. Lysate was then clarified by centrifugation for $1 \mathrm{hr}$ at $4{ }^{\circ} \mathrm{C}$. Clarified lysate was mixed with $1 \mathrm{~mL}$ of ProBond Ni-chelating resin (Thermo Fisher Scientific, Invitrogen \#R80101) per $15 \mathrm{~mL}$ of lysate for $1 \mathrm{hr}$. The resin was washed twice with phosphate buffer $\mathrm{pH} 7$ containing $10 \mu \mathrm{M}$ imidazole, then SUMO-tagged $\mathrm{G} \alpha_{\mathrm{i1}}$ was eluted using the same buffer containing $400 \mathrm{mM}$ imidazole. To remove the SUMO, eluate was dialyzed at $4{ }^{\circ} \mathrm{C}$ in $4 \mathrm{~L}$ of phosphate buffer without imidazole after adding $1 \mathrm{mg}$ ULP1 protease to the dialysis cassette. After dialysis, the cleaved product had no tag and was efficiently purified by reverse metal affinity chromatography. The protein was further purified by passing over a Sepharose $Q$ anion exchange column (GE Healthcare) equilibrated in $100 \mathrm{mM}$ potassium phosphate. The purified yield was typically 3-30 $\mathrm{mg}$ of $\mathrm{G} \alpha / \mathrm{L}$ of cell culture.

Expression and Purification of $\mathbf{G} \beta_{1} \gamma_{2}$. High 5 cells (Invitrogen; $2 \times 10^{6}$ cells $/ \mathrm{mL}$ ) were infected with high titer $\mathrm{G} \beta_{1}$ and $\mathrm{G} \gamma_{2}$ baculoviruses. $\mathrm{G} \beta_{1} \gamma_{2}$ was purified according to (Kozasa and Gilman, 1995), with modifications. All steps were carried out at $4{ }^{\circ} \mathrm{C}$. Cells were harvested $60 \mathrm{hr}$ postinfection by centrifugation at $2600 \mathrm{xg}$ and then resuspended in $50 \mathrm{~mL}$ of lysis buffer $(20 \mathrm{mM}$ HEPES, pH 8, $150 \mathrm{mM} \mathrm{NaCl}, 5 \mathrm{mM}$ 2-mercaptoethanol (2-ME), $1 \mathrm{mM}$ EDTA, $1 \mathrm{~mL}$ of protease inhibitor cocktail (P-2714, Sigma-Aldrich) per liter of cell culture. Cells were lysed by sonication and centrifuged at $2600 \times \mathrm{xg}$ to collect the membranes. Resuspension of membranes were accomplished by dounce homogenization in $100 \mathrm{~mL}$ of lysis buffer. The membranes were solubilized by adding 1\% Lubrol (C12E10; Sigma-Aldrich) with stirring, and the resultant solution was clarified by ultracentrifugation at $125000 \times$ g. The supernatant was loaded onto Ni-NTA agarose (Qiagen) equilibrated with lysis buffer $+1 \%$ Lubrol. The resin was washed and the Lubrol exchanged for sodium cholate using buffers Ni-A (20 mM HEPES, pH 8, $0.4 \mathrm{M} \mathrm{NaCl}, 5$ $\mathrm{mM} 2-\mathrm{ME}, 0.5 \%$ Lubrol, 0.15\% cholate) and Ni-B (20 mM HEPES, pH 8, $0.1 \mathrm{M} \mathrm{NaCl}, 5 \mathrm{mM} 2-$ ME, $0.25 \%$ Lubrol, $0.3 \%$ cholate). G $\beta_{1} \gamma_{2}$ was eluted in Ni-C (20 mM HEPES, pH 8, $0.01 \mathrm{M}$ $\mathrm{NaCl}, 5 \mathrm{mM}$ 2-ME, 1\% cholate, $200 \mathrm{mM}$ imidazole). The purified yield was typically $1 \mathrm{mg}$ of $\mathrm{G} \beta_{1} \gamma_{2} / \mathrm{L}$ of cell culture (Kozasa and Gilman, 1996).

Thermostability Assays (fQCR). $T_{\mathrm{m}}$ values were determined using the fast Quantitative Cysteine Reactivity (fQCR) assay as described previously (Isom et al., 2013). Briefly, $10 \mu \mathrm{L}$ of $40 \mu \mathrm{M}$ protein was added into 12 strip PCR tubes with $170 \mu \mathrm{L}$ phosphate buffer pH $7+10 \mu \mathrm{L}$ of $1 \mathrm{mM}$ guanine nucleotide (GDP or GTP $\gamma \mathrm{S}$ ) + $10 \mu \mathrm{L}$ of $500 \mathrm{mM}$ 4-fluoro-7-

sulfamoylbenzofurazan (ABDF) for $5 \mathrm{~min}$ on ice. The protein was subjected to a temperature gradient in a standard thermocycler for $3 \mathrm{~min}$. The reaction was quenched with ice cold $0.1 \mathrm{~N}$ (final concentration) $\mathrm{HCl}$. The ABDF reacts with exposed cysteine residues and emits light detected on a PHERAstar (BMG Labtech) plate reader using wavelengths using excitation and emission bandpass filters of 400 and $500 \mathrm{~nm}$ (Isom et al., 2013).

Nucleotide loading. BODIPYFL-GTP $\gamma \mathrm{S}(100 \mathrm{nM})$ was equilibrated in buffer (50 mM HEPES, $10 \mathrm{mM} \mathrm{MgCl}_{2}, 25 \mathrm{mM} \mathrm{NaCl}, \mathrm{pH}$ 7) for $60 \mathrm{sec}$. Purified $\mathrm{G \alpha}$ protein (100 nM) was added to a 1 $\mathrm{mL}$ cuvette. The fluorescence of the BODIPYFL group (502 nm excitation, $511 \mathrm{~nm}$ emission) was measured over $600 \mathrm{sec}$. $20 \mu \mathrm{M}$ (final concentration) unlabeled GTP $\gamma \mathrm{S}$ was then added to 
displace BODIPYFL-GTP $\gamma$ S. All measurements were made with a Perkin-Elmer Luminescence Spectrometer and the FLWinLab software package (Jones et al., 2012; McEwen et al., 2001).

Biolayer Interferometry. Binding between $\mathrm{G} \alpha$ and $\mathrm{G} \beta \gamma$ was determined by Biolayer Interferometry (BLI) using Octet Red96 (Fortebio) as described previously (Seneviratne et al., 2011). Briefly, purified biotinylated $\mathrm{G} \beta \gamma(3 \mathrm{mg} / \mathrm{mL})$ was incubated for $15 \mathrm{~min}$ with streptavidin biosensors in either PBST-NGM $\left(25 \mathrm{mM} \mathrm{KPO}_{4}, \mathrm{pH} 7,50 \mathrm{mM} \mathrm{NaCl}, 0.1 \%\right.$ Tween, and $50 \mu \mathrm{M}$ $\mathrm{GDP}+5 \mathrm{mM} \mathrm{MgCl}_{2}$ ) or PBST-NGA (50 $\mu \mathrm{M}$ GDP, $5 \mathrm{mM} \mathrm{MgCl}_{2}, 30 \mu \mathrm{M} \mathrm{AlCl}_{3}$, and $1 \mathrm{mM} \mathrm{NaF}$ ). Purified $\mathrm{G}_{\alpha_{11}}$ (untagged) was diluted to $3 \mu \mathrm{M}$ or $1 \mu \mathrm{M}$ in PBST-NGM or PBST-NGA and then mixed with $\mathrm{G} \beta \gamma$-loaded sensors for 5 min (association) and then protein-free buffer for 5 min (dissociation) at $25^{\circ} \mathrm{C}$. Nonspecific binding was measured using biosensors that were exposed to buffer alone. Baseline subtraction and $G \beta \gamma$ loading normalization were done in Excel. Kinetic Analysis was done using GraphPad Prism.

NMR sample preparation and spectroscopy. ${ }^{15} \mathrm{~N}$-enriched wildtype and R208S $\mathrm{G} \alpha_{\mathrm{i1}}-\Delta 31$ (PET-SUMO-G $\alpha_{i 1}-\Delta 31$ plasmid) were expressed and purified as described above and as detailed previously (Maly and Crowhurst, 2012). The purified proteins were exchanged into NMR buffer (20 mM sodium phosphate, pH 7.0, $50 \mathrm{mM} \mathrm{NaCl}, 2 \mathrm{mM} \mathrm{MgCl}$, $200 \mu \mathrm{M}$ GDP, 5\% $\mathrm{D}_{2} \mathrm{O}$ ). To exchange GDP to GTP $\gamma \mathrm{S}$, the proteins were gently exchanged into a GDP-free solution with $1 \mathrm{mM} \mathrm{MgCl} 2$ and 10 -fold excess of GTP $\gamma \mathrm{S}$ were added to the protein. The sample was incubated on ice for $30 \mathrm{~min}$ and then $\mathrm{MgCl}_{2}$ concentration was increased to $5 \mathrm{mM}$ for another 30 min. GTP $\gamma$ S-loaded samples were finally exchanged into NMR buffer with $200 \mu \mathrm{M}$ GTP $\gamma$ S. Each NMR sample contained $100 \mu \mathrm{M} G \alpha_{i 1}-\Delta 31$. NMR spectra were acquired at $25^{\circ} \mathrm{C}$ on a Bruker Avance 850 NMR spectrometer. Two-dimensional ${ }^{1} \mathrm{H}-{ }^{15} \mathrm{~N}$ HSQC experiments were recorded with 1024 and 128 complex points in the direct and indirect dimensions, respectively, 44 scans per increment and a recovery delay of $1.0 \mathrm{sec}$. Spectral widths used were 13586.957 $\mathrm{Hz}\left({ }^{1} \mathrm{H}\right)$ and $3015.682\left({ }^{15} \mathrm{~N}\right) \mathrm{Hz}$. Spectra were processed and analyzed using NMRPipe (NIDDK, $\mathrm{NIH}$ ) and Sparky (University of California San Francisco). Backbone assignments for wildtype $\mathrm{G} \alpha_{i 1}-\Delta 31$ were transferred from BMRB 30078 for GDP and 26746 for GTP $\gamma$ S (Goricanec et al., 2016). The backbone assignment for $G \alpha_{i 1}-\Delta 31-R 208 S$ were transferred from the wildype $G \alpha_{i 1}$ $\Delta 31$. All ambiguously correlated or resolved peaks were omitted during the transfer.

Starting structural models and the details of molecular dynamics simulations. The initial coordinates of the heterotrimeric stimulatory and inhibitory human $\mathrm{G}$ proteins, $\mathrm{G} \alpha_{\mathrm{s}}$ and $\mathrm{G} \alpha_{\mathrm{i1}}$, were taken from their crystal structures (PDB IDs: 6EG8 and 1GP2 respectively) in the inactive GDP-bound states (Liu et al., 2019; Wall et al., 1995). The initial coordinates of both GTP $\gamma$ S nucleotide as well as the counterion $\mathrm{Mg}^{2+}$ were obtained from the crystal structures of the two $\mathrm{G}$ proteins in the active state (PDB IDs: 1AZT and 1GIA for $\mathrm{G} \alpha_{s}$ and $\mathrm{G} \alpha_{i 1}$ respectively) (Coleman et al., 1994; Sunahara et al., 1997). For building the GTP $\gamma \mathrm{S} / \mathrm{Mg}^{2+}$-bound heterotrimeric $\mathrm{G}$ proteins of each kind the two crystal structures of opposite states were overlaid and GDP/Mg ${ }^{2+}$ in the inactive states were swapped by the GTP $\mathrm{S} / \mathrm{Mg}^{2+}$ in the heterotrimeric states. We removed the N-terminus from both the GDP and the GTP $\gamma \mathrm{S}$ bound G proteins since this region was found to be missing in the monomeric active state crystals of both $\mathrm{G}$ proteins. The GTP $\mathrm{S}$ bound heterotrimeric $G$ proteins of each kind were simulated to capture the early events of dissociation in the $G \alpha$ and $G \beta$ subunits upon replacing GDP with GTP $\gamma$ S. The GDP-bound $G$ proteins in their resting (inactive) states were also simulated as controls. We used CHARMMGUI (Jo et al., 2008) for creating input structures for simulating $G$ proteins ( $G$ s and $G i$ ) in aqueous medium. The nucleotides (GDP and GTP $\gamma \mathrm{S}$ ) and the counterions $\left(\mathrm{Mg}^{2+}\right)$ were parameterized using the CGENFF (Vanommeslaeghe et al., 2012) force field as implemented in 
CHARMM. Point mutations either disrupting or fostering the proposed triad network in Gproteins were incorporated using the MAESTRO software in Schrodinger (https://www.schrodinger.com/maestro). Each of these heterotrimeric protein-nucleotide complexes was solvated in explicit TIP3P water molecules in a cubic box (approximate dimension of $11.30 \mathrm{~nm} \times 11.30 \mathrm{~nm} \times 11.30 \mathrm{~nm}$ ) separately and sodium and chloride counterions were added for maintaining the physiological salt concentration of each system at $150 \mathrm{mM}$. We used the software GROMACS (Hess et al., 2008) (version 2019.4) in combination with the all-atom CHARMM36 (Brooks et al., 2009) force field for performing MD simulations at $310 \mathrm{~K}$ coupled to a temperature bath with a relaxation time of $0.1 \mathrm{ps}$ (Berendsen et al., 1984). Pressure was calculated using molecular virial and held constantly by weak coupling to a pressure bath with a relaxation time of 0.5 ps. Each system was first subjected to a 5000 step steepest descent energy minimization for removing bad contacts (Petrova and Solov'ev, 1997). Then, the systems were heated for $100 \mathrm{ps}$ in steps of ramping up the temperature to $310^{\circ} \mathrm{K}$ under constant temperature-volume ensemble (NVT). Equilibrium bond length and geometry of water molecules were constrained using the SHAKE algorithm (Andersen, 1983). We used a time step of $2 \mathrm{fs}$. The short range electrostatic and van der Waals (VDW) interactions were estimated per time step using a charge group pair list with cut-off radius of $8 \AA$ between the centers of geometry of the charged groups. Long range VDW interactions were calculated using a cut-off of $14 \AA$ and long-range electrostatic interactions were treated using the particle mesh Ewald (PME) method (Darden et al., 1993). Temperature was kept constant by applying the Nose-Hoover thermostat (Evans and Holian, 1985). Parrinello-Rahman barostat (Parrinello and Rahman, 1981) with a pressure relaxation time of 2 ps was used for attaining the desired pressure for all simulations. The simulation trajectories were saved each 200 ps for analysis. The protein, nucleotide and counterion atoms were position restrained using a harmonic force constant of $1000 \mathrm{~kJ}$ mol-1 nm-2 during the NVT equilibration stage while the water molecules were allowed to move freely around the protein. The system was further equilibrated using the constant pressure NPT ensemble by reducing the force constant on protein, counterion and nucleotide atoms from $5 \mathrm{~kJ} \mathrm{~mol}^{-1} \mathrm{~nm}^{-2}$ to zero in a gradual manner for $3 \mathrm{~ns}$ each while having the pressure coupling on. We also performed an additional $10 \mathrm{~ns}$ of unrestrained simulation before beginning the actual production run. This accounts for a total 25 ns of NPT equilibration prior to the production run. We performed three productions runs each 400 ns long starting from three independent sets of initial velocities for each system. Three independent simulations (each 400 ns long) were performed for each system. Thus, we had $1.2 \mu$ s long MD trajectory for each of the wildtype $G \alpha_{s}$ and $G \alpha_{i 1}$ bound to GDP and GTP $\gamma$, four $G \alpha_{i 1}$ mutants (R208S, R208Q, E245K and G203A) and four $G \alpha_{s}$ mutants (R231S, R231Q, E268K and G226A) bound to GTP $\gamma$ S.

Calculation of root mean square fluctuations (RMSF). Per residue RMSF for each $G \alpha_{i 1}$ system was computed using the gmx rmsf utility with -res option as implemented in GROMACS. The per residue RMSFs were inserted as B-factor columns of the representative snapshots of the $\mathrm{G} \alpha$ subunits of each $\mathrm{G} \alpha_{i 1}$ system and shown as cartoons.

Calculation of interaction energy. Short range electrostatic and van der Waals interactions were estimated per time step using a charge group pair list with cut-off radius of $8 \AA$ between the centers of geometry of the charged groups. The residue pairs of the $G$ proteins engaged in van der Waals or electrostatic interactions for $>40 \%$ of the whole simulation were considered as sustained contacts and were identified using the GetContacts script available at the GitHub (https://getcontacts.github.io/). These residues of interest were indexed and tagged as energy groups. The total non-bonded interactions energies (van der Waals + Coulombic) between two protein subunits, $\mathrm{G} \alpha$ and $\mathrm{G} \beta$ or between $\mathrm{G} \alpha$ and nucleotide were extracted using the gmx 
energy module of GROMACS. The energies were calculated for each snapshot and averaged over the last $300 \mathrm{~ns}$ in each of the three simulation runs for each system. For calculating the interaction energies between Switch II and Switch III regions we used the following definition of Switch II and Switch III regions. Residues G203 to E216 make up the Switch II region for $\mathrm{G} \alpha_{i 1}$ and residues $\mathrm{G} 226$ to $\mathrm{N} 239$ for $\mathrm{G} \alpha_{\mathrm{s}}$. Switch III is comprised of residues E236 to N255 for G $\alpha_{i 1}$ and E259 to $\mathrm{N} 278$ for $\mathrm{G} \alpha_{\mathrm{s}}$.

Representative structure calculation from MD simulation trajectories. Representative snapshots from the most occupied conformation cluster for each system were chosen for structure representation in the figures. The conformation clustering of the snapshots in the MD trajectories was done using the RMSD-based clustering method using the GROMACS modules gmx rms and gmx cluster with a $1.5 \AA$ cutoff on the concatenated trajectory from three simulations (a total of $1.2 \mu$ s simulation time) for each system. The most representative structure of the most populated cluster was calculated as the frame that has the smallest RMSD to the center of this cluster conformation. Snapshots were rendered using VMD (Humphrey et al., 1996) and PYMOL (https://www.schrodinger.com/pymol).

Principal component analysis and free energy surface generation. For each system in this study, we merged the three independent MD runs into one concatenated trajectory. We then performed the principal component analysis using the gmx covar module of GROMACS for each system using covariance matrix of the $\mathrm{C} \alpha$ atoms of all of the residues. The gmx sham module of GROMACS was used to compute the probability of the microstates and convert it to free energy. The free energy surface thus generated was projected on the principal component space covered by principal component 1 and 2 (PC1 and PC2). We then clustered the MD snapshots by the values of PC1 and PC2 using the in-house extractcluster.py script in MATLAB and calculated the population of each of these conformational clusters (Figure 4). The population of the most populated cluster 1 was compared across the various systems under consideration for $\mathrm{G} \alpha_{i 1}$ (Figure S4, Supplemental Information). The MD snapshots under each conformational cluster were concatenated to obtain trajectories for each of these clusters and then the VMD script helicity.tcl was used to calculate the helicity of the Switch II regions in $G \alpha_{i 1}$. The cumulative fraction of variance in the eigenvalues of the PCs (shown in Figure S4, Supplementary Information) accounts for about $95 \%$ of the variance captured by the first ten PCs for each $\mathrm{G}_{\mathrm{i1}}$ system. 


\section{SUPPLEMENTAL FIGURE LEGENDS}

Figure S1 (Related to Figure 1). Multiple sequence alignment.

Benchling software was used to align human $\mathrm{G} \alpha$ subunits as well as Arabidopsis AtGPA1 and yeast Gpa1. Triad residues are indicated by red boxes. Crystal model of $G \alpha_{s}, G \alpha_{i 1}, G \alpha_{t} G \alpha_{13}$, $\mathrm{G} \alpha_{\mathrm{q}}$, AtGPA1 triad residues and GTP $\gamma \mathrm{S}$ or $\mathrm{GDP}$ AlF $_{4}{ }^{-}$(ball and stick) from the indicated PDB structures.

\section{Figure S2 (Related to Figure 2). Linked Excel primer table}

\section{Figure S3 (Related to Figure 3). G $\alpha_{s}$ network analysis}

(A) A dynamic mechanism for the effect of the $\gamma$-phosphate group triggering the release of the G $\beta \gamma$ subunits from $G \alpha_{s}$. The dynamic pull-push effect from the $\gamma$-phosphate group of GTP $\gamma$, that forms the polar residue interaction network involving the P-loop, Switch I, Switch II and Switch III residues, derived from MD simulations for wildtype $G \alpha_{s}$ bound to GTP $\gamma S$ (top) or GDP (bottom). The nucleotides are shown in ball and stick representation. The blue and the red dotted lines indicate sustained and broken residue contacts, respectively. Right panels show residues involved in the polar network for the GTP $\gamma$ S- and GDP-bound G $\alpha_{i 1}$ switch regions (black and gray colors respectively).

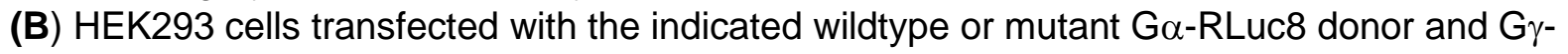
GFP acceptor proteins. Concentration-response measurements $(n=2)$ using $\mu$-opioid receptor are presented as fold-decrease in dynamic range (dissociation, Net BRET).

(C) A dynamic mechanism for the effect of the $\gamma$-phosphate group triggering the release of the G $\beta \gamma$ subunits from $\mathrm{G} \alpha_{i 1}$ R208S (red), E245K (blue), G203A (purple), and R208Q (orange). The dynamic pull-push effect from the $\gamma$-phosphate group of GTP $\gamma S$, that forms the polar residue interaction network involving the P-loop, Switch I, Switch II and Switch III residues, derived from MD simulations for wildtype $\mathrm{G} \alpha_{i 1}$ bound to GTP $\gamma \mathrm{S}$.

(D) A dynamic mechanism for the effect of the $\gamma$-phosphate group triggering the release of the $\mathrm{G} \beta \gamma$ subunits from $\mathrm{G} \alpha_{s}$ R231S (red), E264K (blue), G226A (purple), and R231Q (orange). The dynamic pull-push effect from the $\gamma$-phosphate group of GTP $\gamma$, that forms the polar residue interaction network involving the P-loop, Switch I, Switch II and Switch III residues, derived from MD simulations for wildtype $\mathrm{G} \alpha_{s}$ bound to GTP $\gamma$ S.

\section{Figure S4 (Related to Figure 4). MD simulations of triad residues that coordinate the active-state conformation.}

(A) and (B) Root mean square fluctuations (RMSF) indicating the flexibility of the residues in the protein. The heat map of RMSF is projected on the structures of wild type and various triad mutants of $G \alpha_{i}$ bound to either GDP or GTP as indicated in the figures.

(C) The variance in the principal components and its cumulative fraction shown to indicate that the top four principal components carry most of the weight in the principal component analysis that was used to project the free energy surfaces shown in Fig. 4B.

\section{Figure S5 (Related to Figure 5). NMR supplementary spectra}

$2 \mathrm{D}\left[{ }^{15} \mathrm{~N},{ }^{1} \mathrm{H}\right]-\mathrm{HSQC}$ NMR of $\mathrm{G} \alpha_{\mathrm{i} 1} \Delta 31$ wildtype and R208S mutant.

(A) Overlay of R208S G $\alpha_{i 1}$ in complex with GTP $\gamma \mathrm{S}$ (red), and GDP (orange)

(B) Overlay of wildtype (blue) and R208S (orange) $G \alpha_{i 1}$ each in complex with GDP 


\section{REFERENCES}

Andersen, H.C. (1983). Rattle: A "Velocity" Version of the Shake Algorithm for Molecular Dynamics Calculations. Journal of Computational Physics 52, 24-34.

Apanovitch, D.M., liri, T., Karasawa, T., Bourne, H.R., and Dohlman, H.G. (1998). Second site suppressor mutations of a GTPase-deficient G-protein a- subunit. Selective inhibition of bgmediated signaling. J Biol Chem 273, 28597-28602.

Berendsen, H.J.C., Postma, J.P.M., Gunsteren, W.F.v., DiNola, A., and Haak, J.R. (1984). Molecular dynamics with coupling to an external bath. The Journal of Chemical Physics 81, 3684-3690.

Berghuis, A.M., Lee, E., Raw, A.S., Gilman, A.G., and Sprang, S.R. (1996). Structure of the GDP-Pi complex of Gly203-->Ala gialpha1: a mimic of the ternary product complex of galpha-catalyzed GTP hydrolysis. Structure 4, 1277-1290.

Bourne, H.R., Kaslow, D., Kaslow, H.R., Salomon, M.R., and Licko, V. (1981). Hormone-sensitive adenylate cyclase. Mutant phenotype with normally regulated beta-adrenergic receptors uncoupled with catalytic adenylate cyclase. Mol Pharmacol 20,435-441.

Brooks, B.R., Brooks III, C.L., Mackerell Jr., A.D., Nilsson, L., Petrella, R.J., Roux, B., Won, Y., Archontis, G., Bartels, C., Boresch, S., et al. (2009). CHARMM: The biomolecular simulation program. Journal of Computational Chemistry 30, 1545-1614.

Che, T., English, J., Krumm, B.E., Kim, K., Pardon, E., Olsen, R.H.J., Wang, S., Zhang, S., Diberto, J.F., Sciaky, N., et al. (2020). Nanobody-enabled monitoring of kappa opioid receptor states. Nat Commun 11, 1145.

Chung, K.Y., Rasmussen, S.G., Liu, T., Li, S., DeVree, B.T., Chae, P.S., Calinski, D., Kobilka, B.K., Woods, V.L., Jr., and Sunahara, R.K. (2011). Conformational changes in the G protein Gs induced by the beta2 adrenergic receptor. Nature 477, 611-615.

Coleman, D.E., Berghuis, A.M., Lee, E., Linder, M.E., Gilman, A.G., and Sprang, S.R. (1994). Structures of active conformations of Gi alpha 1 and the mechanism of GTP hydrolysis. Science $265,1405-1412$.

Darden, T., York, D., and Pedersen, L. (1993). Particle mesh Ewald: An N·log(N) method for Ewald sums in large systems. The Journal of Chemical Physics 98, 10089-10092.

De Lean, A., Stadel, J.M., and Lefkowitz, R.J. (1980). A ternary complex model explains the agonist-specific binding properties of the adenylate cyclase-coupled beta-adrenergic receptor. J Biol Chem 255, 7108-7117. 
DeVree, B.T., Mahoney, J.P., Velez-Ruiz, G.A., Rasmussen, S.G., Kuszak, A.J., Edwald, E., Fung, J.J., Manglik, A., Masureel, M., Du, Y., et al. (2016). Allosteric coupling from $G$ protein to the agonist-binding pocket in GPCRs. Nature 535, 182-186.

Dowell, S.J., and Brown, A.J. (2009). Yeast assays for $G$ protein-coupled receptors. Methods Mol Biol 552, 213-229.

Draper-Joyce, C.J., Khoshouei, M., Thal, D.M., Liang, Y.L., Nguyen, A.T.N., Furness, S.G.B., Venugopal, H., Baltos, J.A., Plitzko, J.M., Danev, R., et al. (2018). Structure of the adenosinebound human adenosine A1 receptor-Gi complex. Nature 558, 559-563.

Dror, R.O., Mildorf, T.J., Hilger, D., Manglik, A., Borhani, D.W., Arlow, D.H., Philippsen, A., Villanueva, N., Yang, Z., Lerch, M.T., et al. (2015). Structural basis for nucleotide exchange in heterotrimeric G proteins. Science 348, 1361-1365.

Evans, D.J., and Holian, B.L. (1985). The Nose-Hoover thermostat. The Journal of Chemical Physics 83, 4069-4074.

Farfel, Z., liri, T., Shapira, H., Roitman, A., Mouallem, M., and Bourne, H.R. (1996).

Pseudohypoparathyroidism, a novel mutation in the betagamma-contact region of Gsalpha impairs receptor stimulation. J Biol Chem 271, 19653-19655.

Flock, T., Hauser, A.S., Lund, N., Gloriam, D.E., Balaji, S., and Babu, M.M. (2017). Selectivity determinants of GPCR-G-protein binding. Nature 545, 317-322.

Flock, T., Ravarani, C.N.J., Sun, D., Venkatakrishnan, A.J., Kayikci, M., Tate, C.G., Veprintsev, D.B., and Babu, M.M. (2015). Universal allosteric mechanism for Galpha activation by GPCRs. Nature 524, 173-179.

Ghosh, S., Bierig, T., Lee, S., Jana, S., Lohle, A., Schnapp, G., Tautermann, C.S., and Vaidehi, N. (2018). Engineering Salt Bridge Networks between Transmembrane Helices Confers Thermostability in G-Protein-Coupled Receptors. J Chem Theory Comput 14, 6574-6585.

Gilman, A.G. (1987). G proteins: transducers of receptor-generated signals. Annu Rev Biochem $56,615-649$.

Goricanec, D., Stehle, R., Egloff, P., Grigoriu, S., Pluckthun, A., Wagner, G., and Hagn, F. (2016). Conformational dynamics of a G-protein alpha subunit is tightly regulated by nucleotide binding. Proc Natl Acad Sci U S A 113, E3629-3638.

Hess, B., Kutzner, C., van der Spoel, D., and Lindahl, E. (2008). GROMACS 4: Algorithms for Highly Efficient, Load-Balanced, and Scalable Molecular Simulation. J Chem Theory Comput 4, 435-447. 
Hoffman, G., Garrison, T.R., and Dohlman, H.G. (2002). Analysis of RGS proteins in Saccharomyces cerevisiae. Methods Enzymol 344, 617-631.

Humphrey, W., Dalke, A., and Schulten, K. (1996). VMD: Visual molecular dynamics. Journal of Molecular Graphics 14, 33-38.

liri, T., Farfel, Z., and Bourne, H.R. (1997). Conditional activation defect of a human Gsalpha mutant. Proc Natl Acad Sci U S A 94, 5656-5661.

Isom, D.G., Sridharan, V., Baker, R., Clement, S.T., Smalley, D.M., and Dohlman, H.G. (2013). Protons as second messenger regulators of $\mathrm{G}$ protein signaling. Mol Cell 51, 531-538.

Jo, S., Kim, T., Iyer, V.G., and Im, W. (2008). CHARMM-GUI: a web-based graphical user interface for CHARMM. J Comput Chem 29, 1859-1865.

Jones, J.C., Jones, A.M., Temple, B.R., and Dohlman, H.G. (2012). Differences in intradomain and interdomain motion confer distinct activation properties to structurally similar Ga proteins. Proc Natl Acad Sci U S A.

Koehl, A., Hu, H., Maeda, S., Zhang, Y., Qu, Q., Paggi, J.M., Latorraca, N.R., Hilger, D., Dawson, R., Matile, H., et al. (2018). Structure of the micro-opioid receptor-Gi protein complex. Nature 558, 547-552.

Kozasa, T., and Gilman, A.G. (1995). Purification of recombinant G proteins from Sf9 cells by hexahistidine tagging of associated subunits. Characterization of alpha 12 and inhibition of adenylyl cyclase by alpha z. J Biol Chem 270, 1734-1741.

Kozasa, T., and Gilman, A.G. (1996). Protein kinase C phosphorylates G12 alpha and inhibits its interaction with $\mathrm{G}$ beta gamma. J Biol Chem 271, 12562-12567.

Lambright, D.G., Noel, J.P., Hamm, H.E., and Sigler, P.B. (1994). Structural determinants for activation of the alpha-subunit of a heterotrimeric $G$ protein [see comments]. Nature 369, 621628.

Lambright, D.G., Sondek, J., Bohm, A., Skiba, N.P., Hamm, H.E., and Sigler, P.B. (1996). The 2.0 A crystal structure of a heterotrimeric $G$ protein [see comments]. Nature $379,311-319$.

Lee, E., Taussig, R., and Gilman, A.G. (1992). The G226A mutant of Gs alpha highlights the requirement for dissociation of $\mathrm{G}$ protein subunits. J Biol Chem 267, 1212-1218.

Liang, Y.L., Khoshouei, M., Deganutti, G., Glukhova, A., Koole, C., Peat, T.S., Radjainia, M., Plitzko, J.M., Baumeister, W., Miller, L.J., et al. (2018a). Cryo-EM structure of the active, Gsprotein complexed, human CGRP receptor. Nature 561, 492-497. 
Liang, Y.L., Khoshouei, M., Glukhova, A., Furness, S.G.B., Zhao, P., Clydesdale, L., Koole, C., Truong, T.T., Thal, D.M., Lei, S., et al. (2018b). Phase-plate cryo-EM structure of a biased agonist-bound human GLP-1 receptor-Gs complex. Nature 555, 121-125.

Liu, X., Xu, X., Hilger, D., Aschauer, P., Tiemann, J.K.S., Du, Y., Liu, H., Hirata, K., Sun, X., GuixaGonzalez, R., et al. (2019). Structural Insights into the Process of GPCR-G Protein Complex Formation. Cell 177, 1243-1251 e1212.

Maeda, S., Koehl, A., Matile, H., Hu, H., Hilger, D., Schertler, G.F.X., Manglik, A., Skiniotis, G., Dawson, R.J.P., and Kobilka, B.K. (2018). Development of an antibody fragment that stabilizes GPCR/G-protein complexes. Nat Commun 9, 3712.

Maly, J., and Crowhurst, K.A. (2012). Expression, purification and preliminary NMR characterization of isotopically labeled wild-type human heterotrimeric $\mathrm{G}$ protein alphai1. Protein Expr Purif 84, 255-264.

McEwen, D.P., Gee, K.R., Kang, H.C., and Neubig, R.R. (2001). Fluorescent BODIPY-GTP analogs: real-time measurement of nucleotide binding to G proteins. Anal Biochem 291, 109-117.

Miller, R.T., Masters, S.B., Sullivan, K.A., Beiderman, B., and Bourne, H.R. (1988). A mutation that prevents GTP-dependent activation of the alpha chain of Gs. Nature 334, 712-715.

Mixon, M.B., Lee, E., Coleman, D.E., Berghuis, A.M., Gilman, A.G., and Sprang, S.R. (1995). Tertiary and quaternary structural changes in Gi alpha 1 induced by GTP hydrolysis [see comments]. Science 270, 954-960.

Olsen, R.H.J., DiBerto, J.F., English, J.G., Glaudin, A.M., Krumm, B.E., Slocum, S.T., Che, T., Gavin, A.C., McCorvy, J.D., Roth, B.L., et al. (2020). TRUPATH, an open-source biosensor platform for interrogating the GPCR transducerome. Nat Chem Biol.

Parrinello, M., and Rahman, A. (1981). Polymorphic transitions in single crystals: A new molecular dynamics method. Journal of Applied Physics 52, 7182-7190.

Petrova, S.S., and Solov'ev, A.D. (1997). The Origin of the Method of Steepest Descent. Historia Mathematica 24, 361-375.

Rasmussen, S.G., DeVree, B.T., Zou, Y., Kruse, A.C., Chung, K.Y., Kobilka, T.S., Thian, F.S., Chae, P.S., Pardon, E., Calinski, D., et al. (2011). Crystal structure of the beta2 adrenergic receptor-Gs protein complex. Nature 477, 549-555.

Salomon, M.R., and Bourne, H.R. (1981). Novel S49 lymphoma variants with aberrant cyclic AMP metabolism. Mol Pharmacol 19, 109-116. 
Seneviratne, A.M., Burroughs, M., Giralt, E., and Smrcka, A.V. (2011). Direct-reversible binding of small molecules to $G$ protein betagamma subunits. Biochim Biophys Acta 1814, 1210-1218.

Shellhammer, J.P., Pomeroy, A.E., Li, Y., Dujmusic, L., Elston, T.C., Hao, N., and Dohlman, H.G. (2019). Quantitative analysis of the yeast pheromone pathway. Yeast 36, 495-518.

Sondek, J., Lambright, D.G., Noel, J.P., Hamm, H.E., and Sigler, P.B. (1994). GTPase mechanism of $\mathrm{G}$ proteins from the 1.7-A crystal structure of transducin a-GDP-AIF-4. Nature 372, 276-279.

Song, J., Hirschman, J., Gunn, K., and Dohlman, H.G. (1996). Regulation of membrane and subunit interactions by N-myristoylation of a $\mathrm{G}$ protein a subunit in yeast. J Biol Chem 271, 20273-20283.

Stratton, H.F., Zhou, J., Reed, S.I., and Stone, D.E. (1996). The mating-specific Ga protein of Saccharomyces cerevisiae downregulates the mating signal by a mechanism that is dependent on pheromone and independent of Gbg sequestration. Mol Cell Biol 16, 6325-6337.

Sun, D., Flock, T., Deupi, X., Maeda, S., Matkovic, M., Mendieta, S., Mayer, D., Dawson, R., Schertler, G.F.X., Madan Babu, M., et al. (2015). Probing Galphai1 protein activation at singleamino acid resolution. Nat Struct Mol Biol 22, 686-694.

Sunahara, R.K., Tesmer, J.J., Gilman, A.G., and Sprang, S.R. (1997). Crystal structure of the adenylyl cyclase activator Gsalpha. Science 278, 1943-1947.

Vaidehi, N., Grisshammer, R., and Tate, C.G. (2016). How Can Mutations Thermostabilize GProtein-Coupled Receptors? Trends Pharmacol Sci 37, 37-46.

Van Eps, N., Preininger, A.M., Alexander, N., Kaya, A.I., Meier, S., Meiler, J., Hamm, H.E., and Hubbell, W.L. (2011). Interaction of a G protein with an activated receptor opens the interdomain interface in the alpha subunit. Proc Natl Acad Sci U S A 108, 9420-9424.

Vanommeslaeghe, K., Raman, E.P., and MacKerell, A.D., Jr. (2012). Automation of the CHARMM General Force Field (CGenFF) II: assignment of bonded parameters and partial atomic charges. J Chem Inf Model 52, 3155-3168.

Wall, M.A., Coleman, D.E., Lee, E., Iniguez-Lluhi, J.A., Posner, B.A., Gilman, A.G., and Sprang, S.R. (1995). The structure of the G protein heterotrimer Gi alpha 1 beta 1 gamma 2. Cell 83, 10471058. 
bioRxiv preprint doi: https://doi.org/10.1101/2020.11.15.383679; this version posted November 16,2020 . The copyright holder for this preprint (which was not certified by peer review) is the author/funder. All rights reserved. No reuse allowed without permission. 


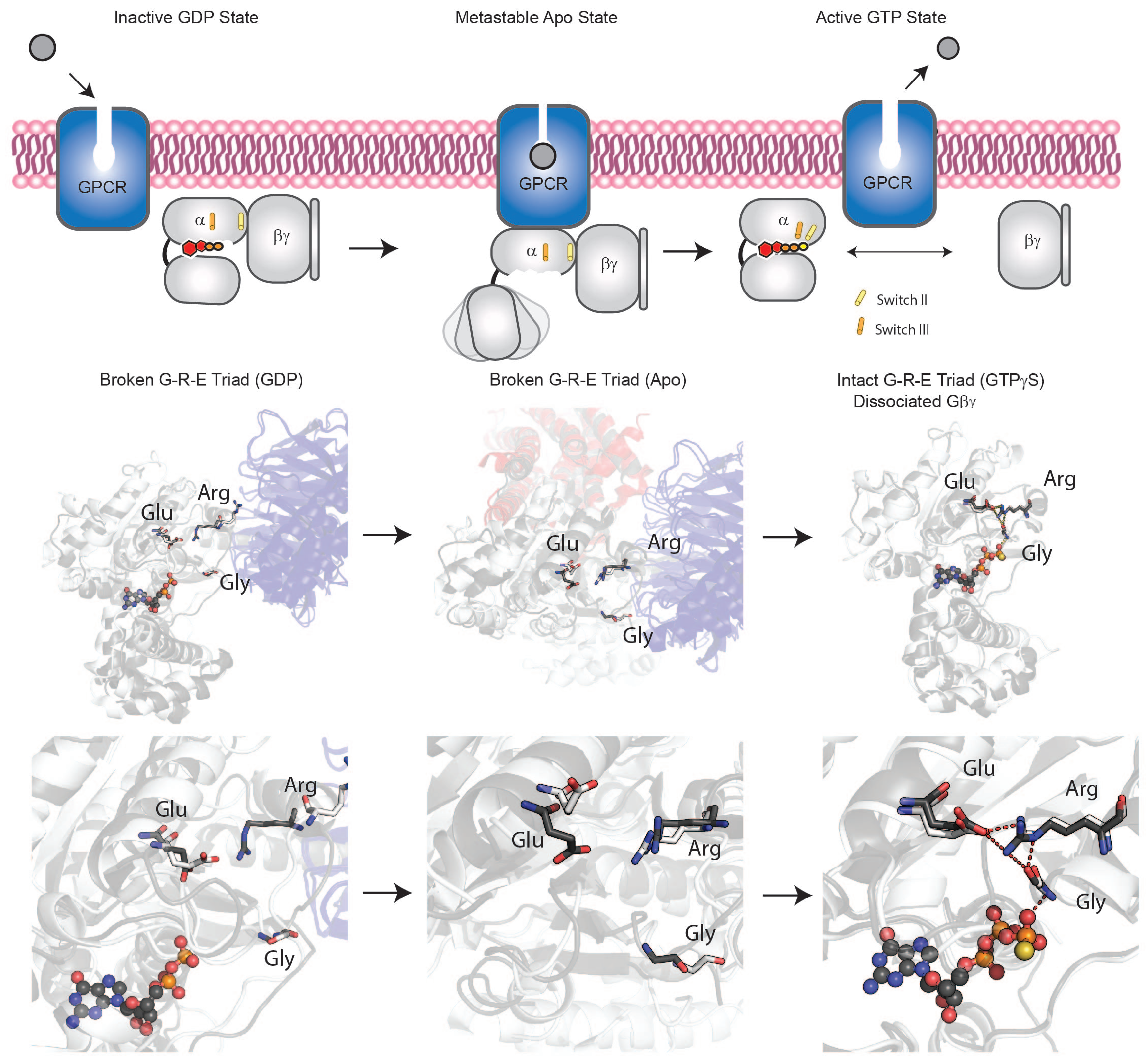



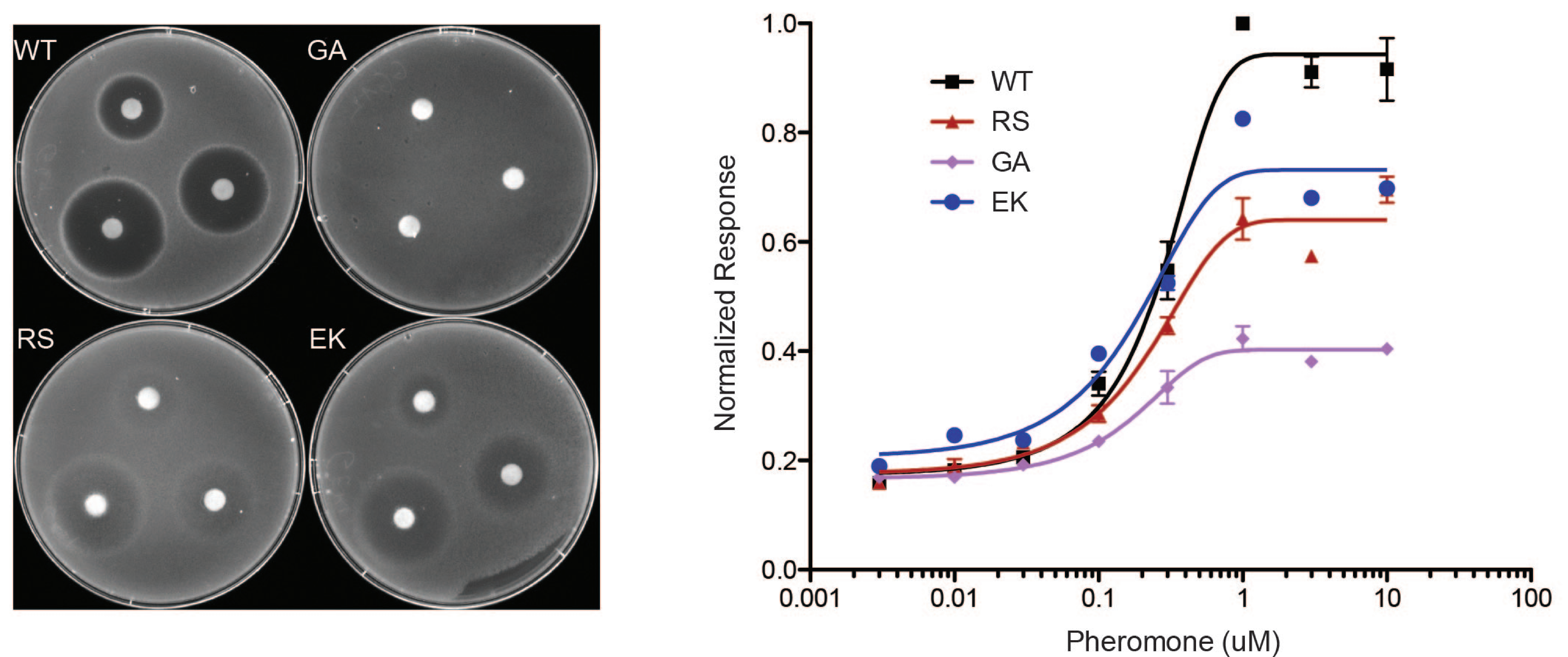

Mutant WT RA RC RD RE RF RG RH RI RK RL RM RN RP RQ RS RT RV RW RY RX

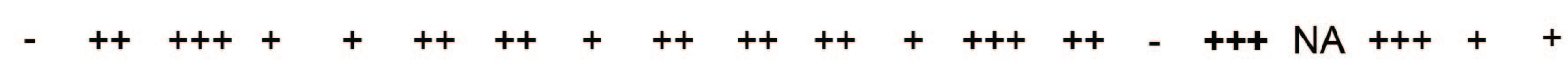


A

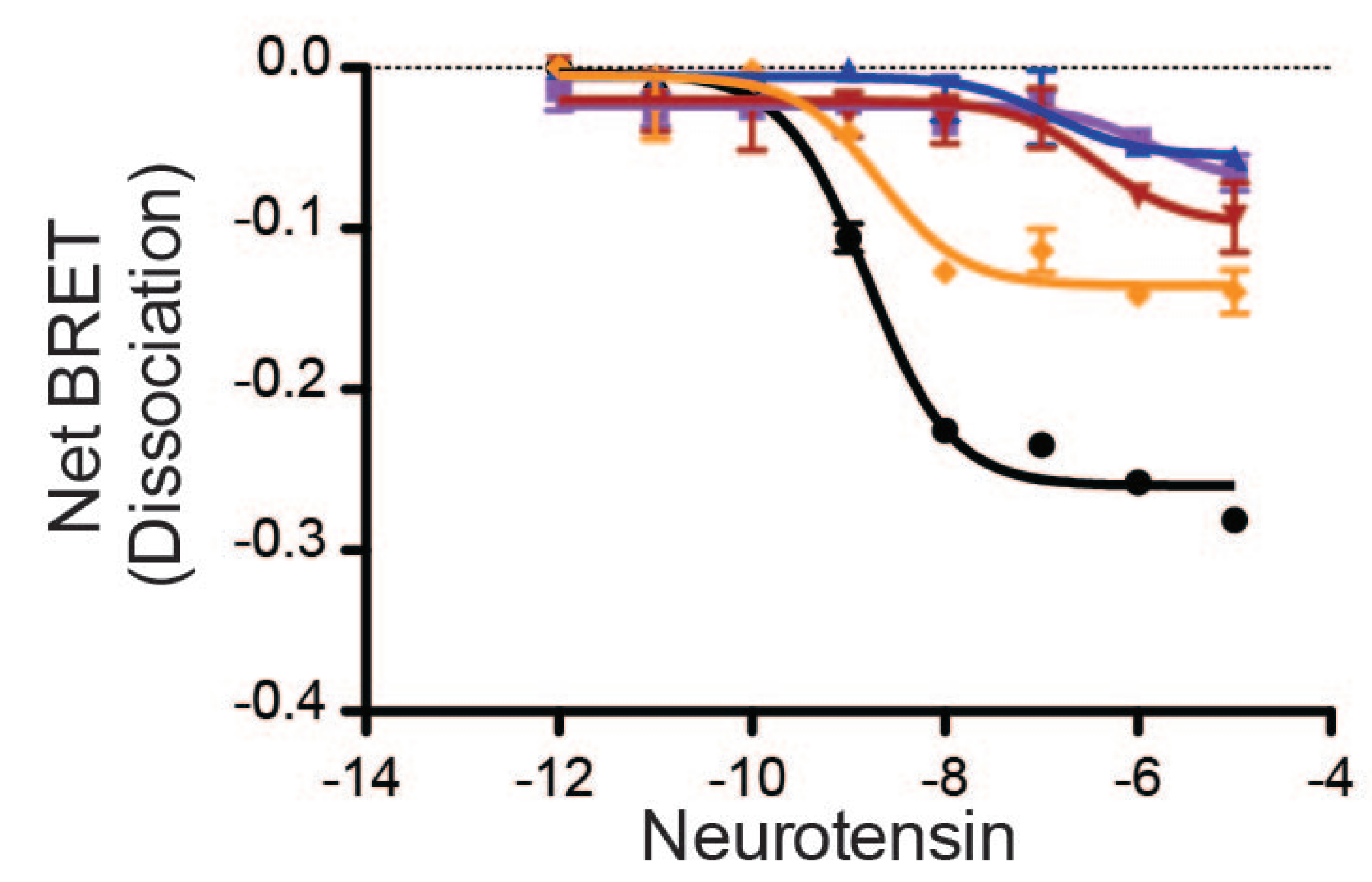

B

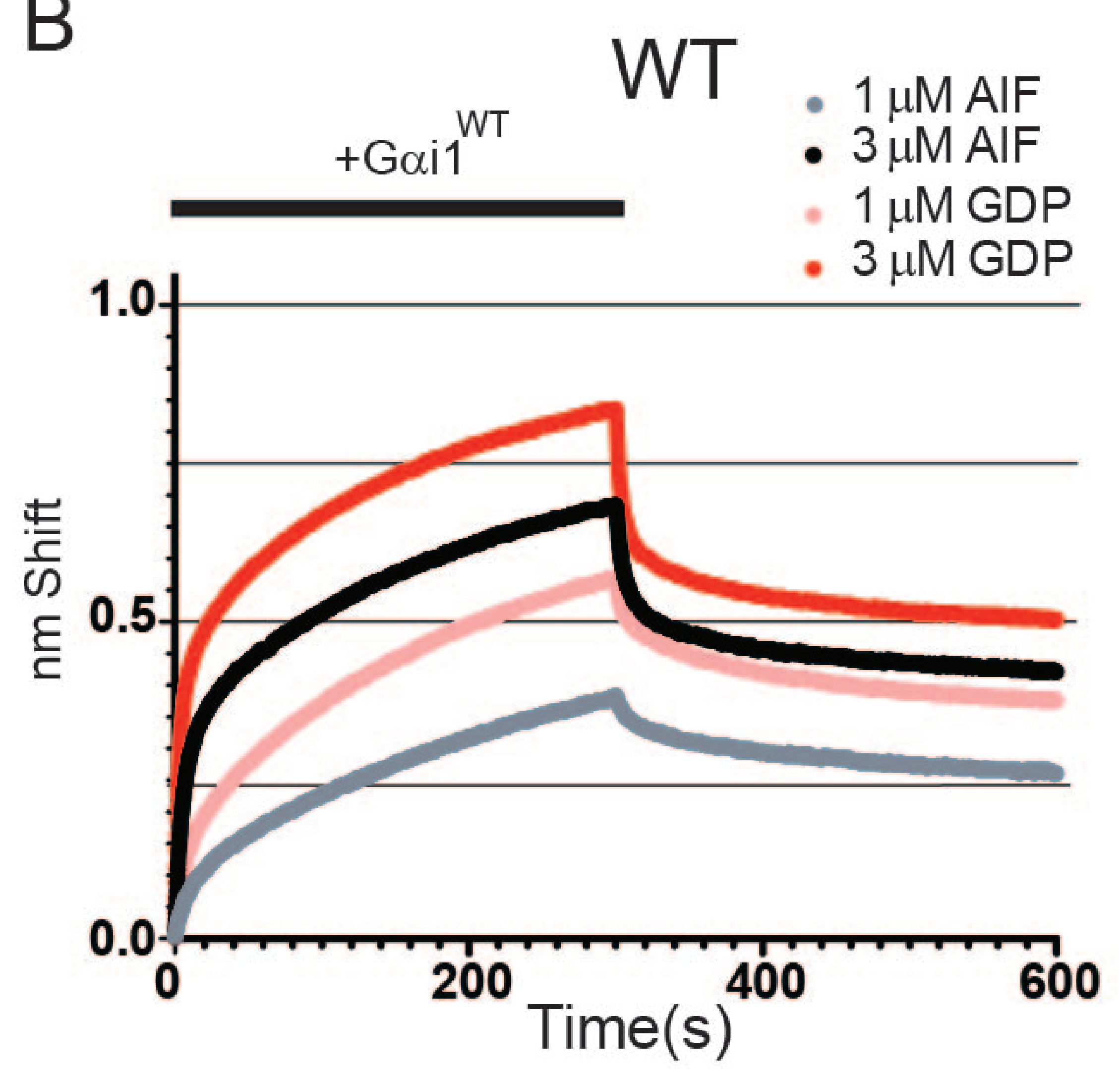

D Contacts forme

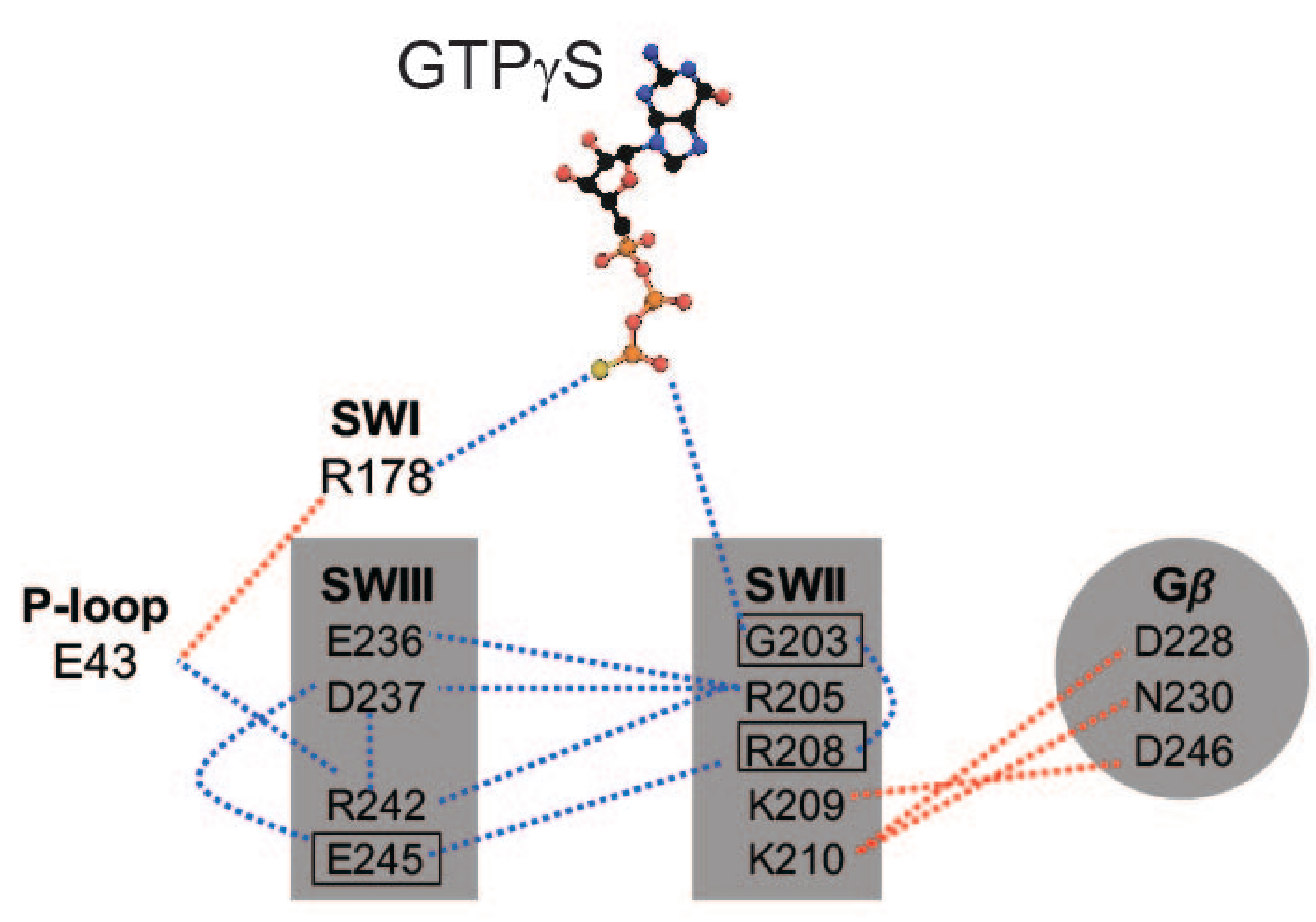

Gs
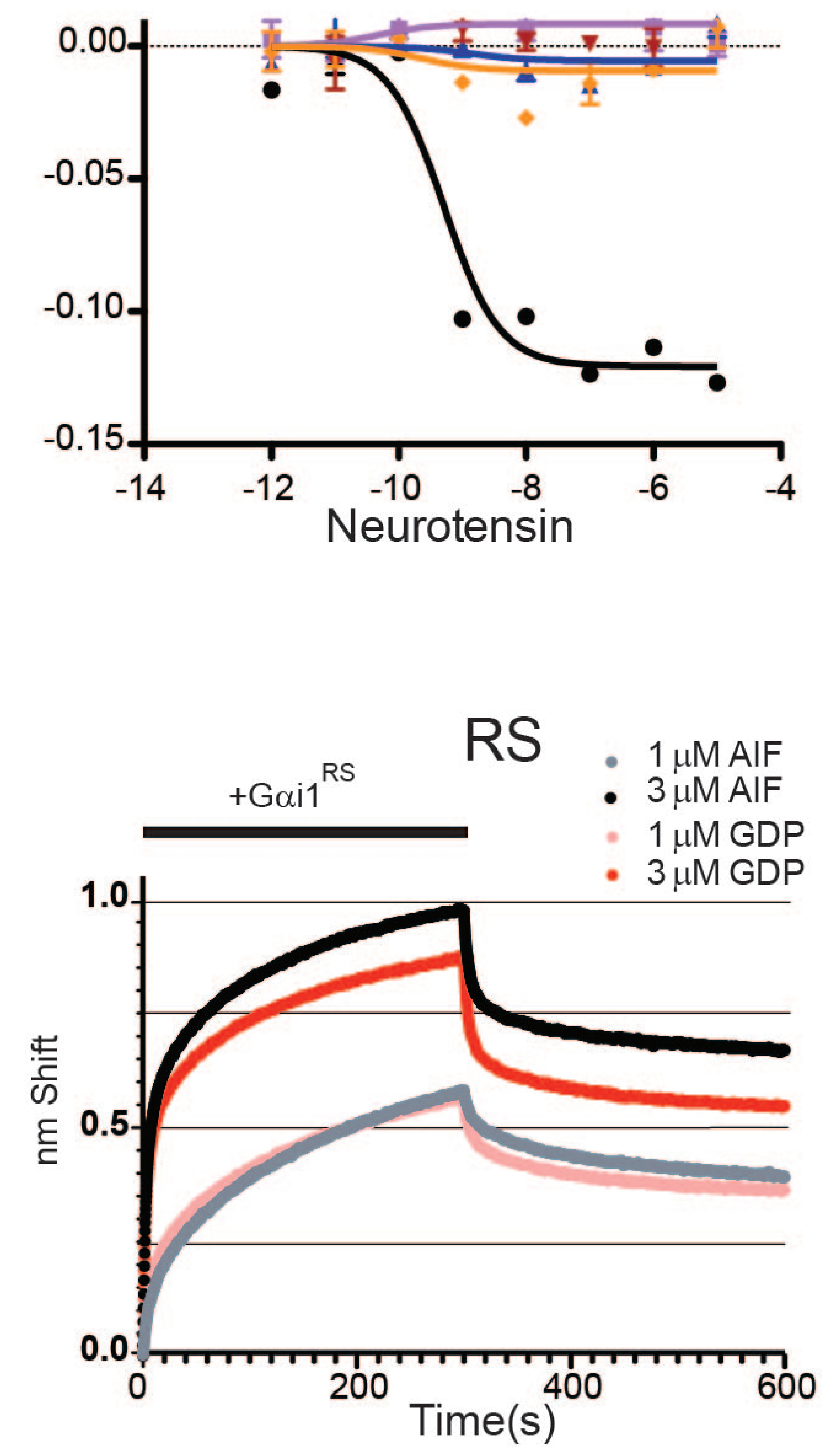

SWII-SWIII Interface (GTP $\gamma$ S)

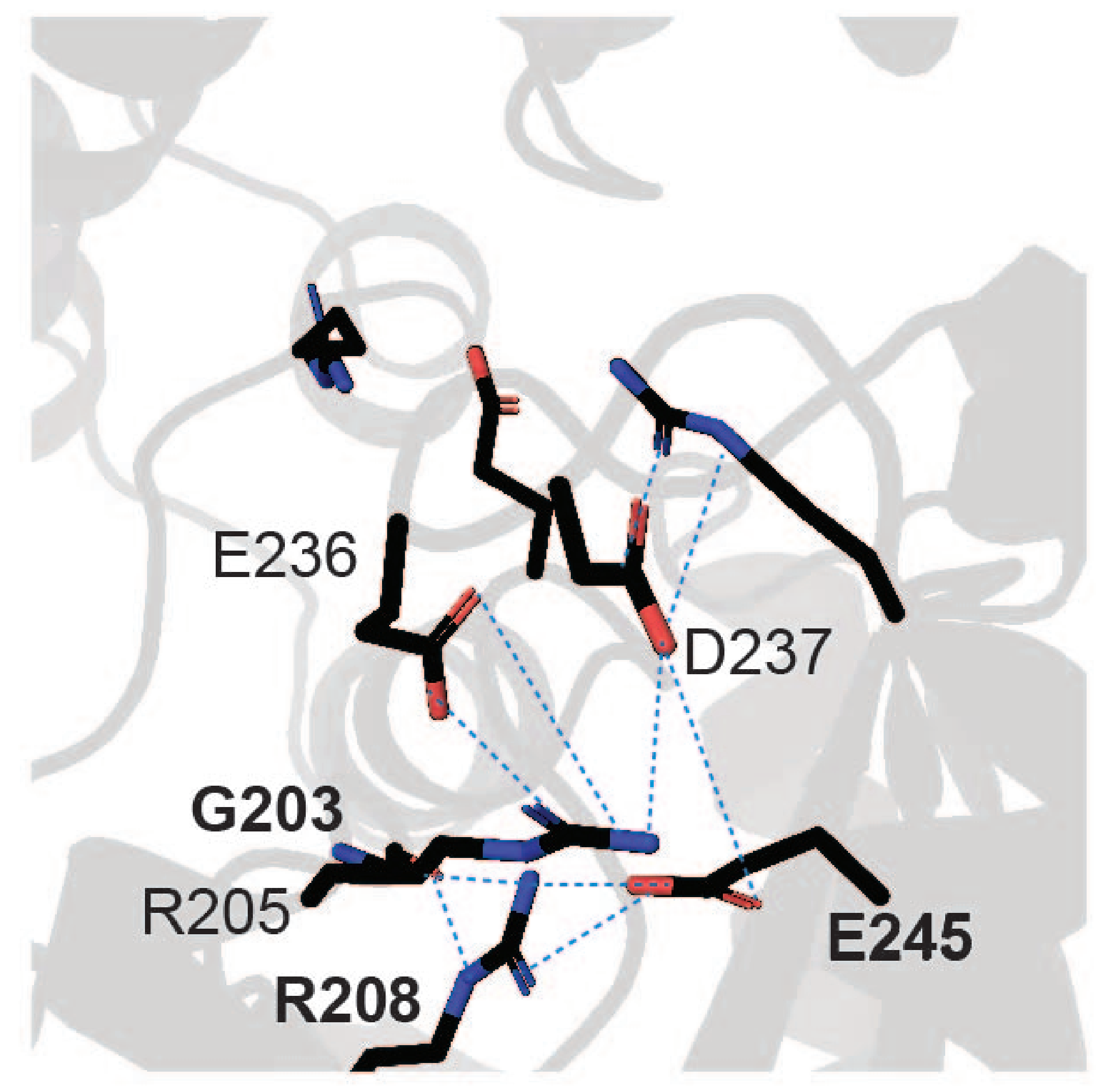

SWII-SWIII Interface (GDP)

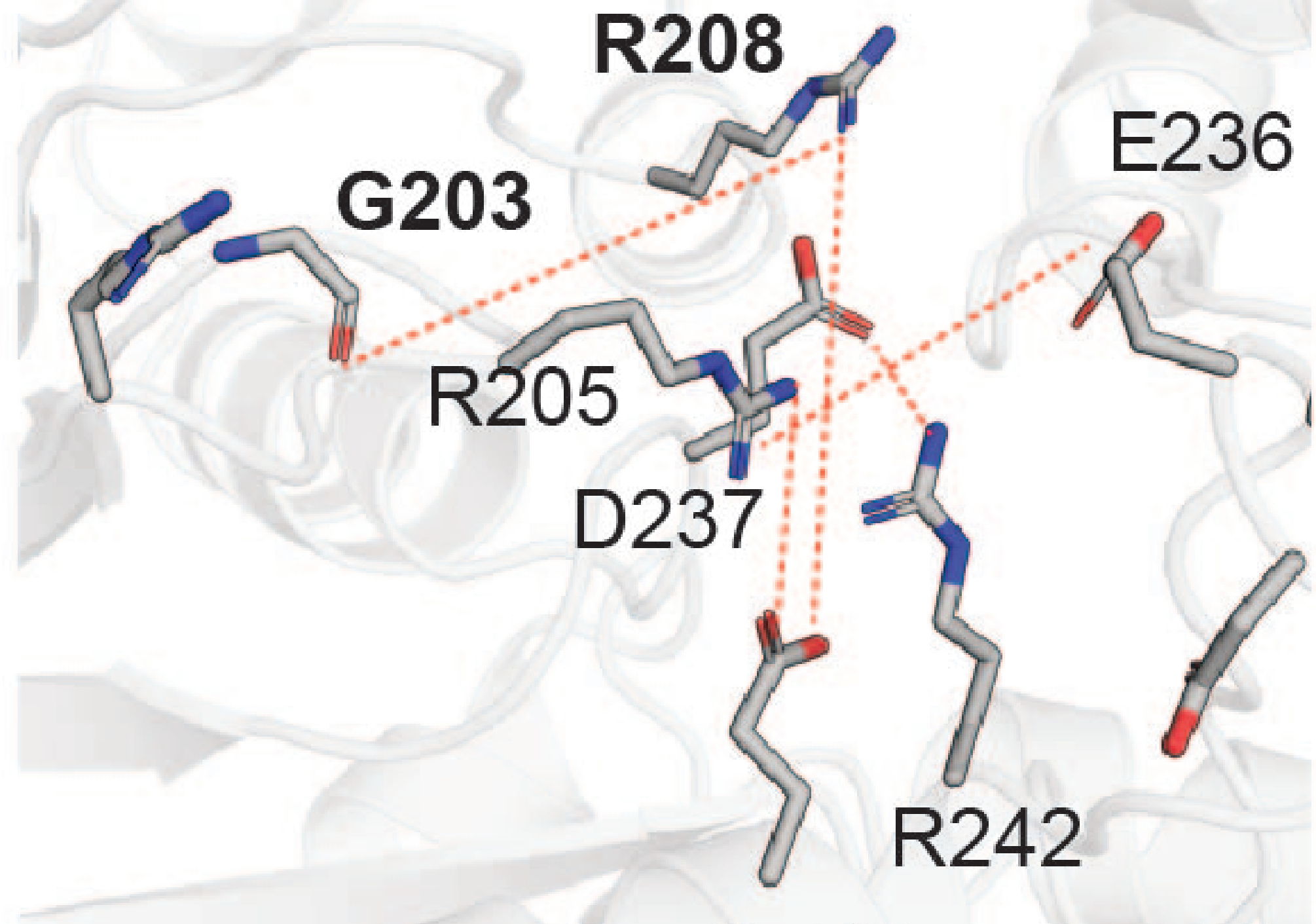

$\mathrm{Gq}$

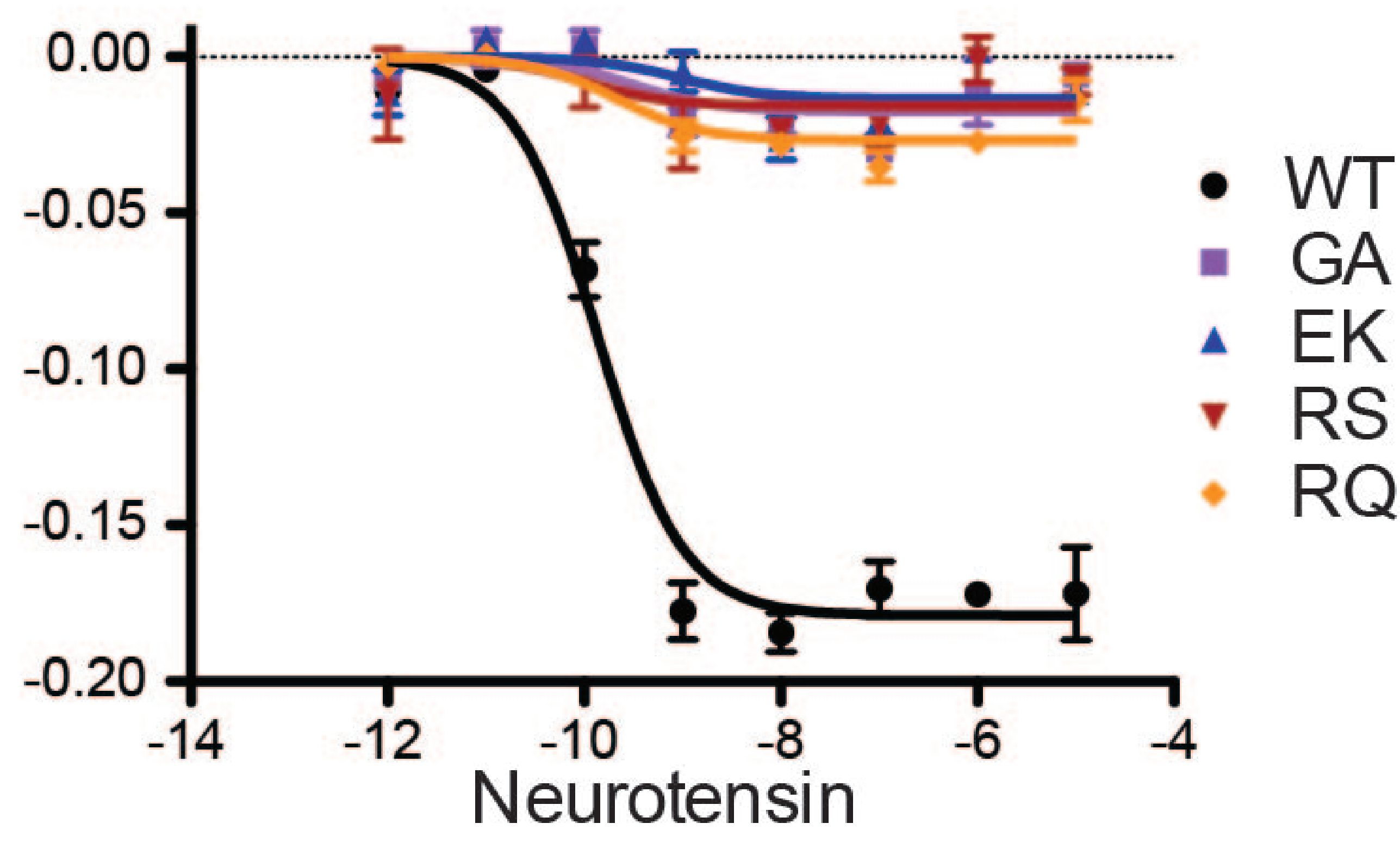

C

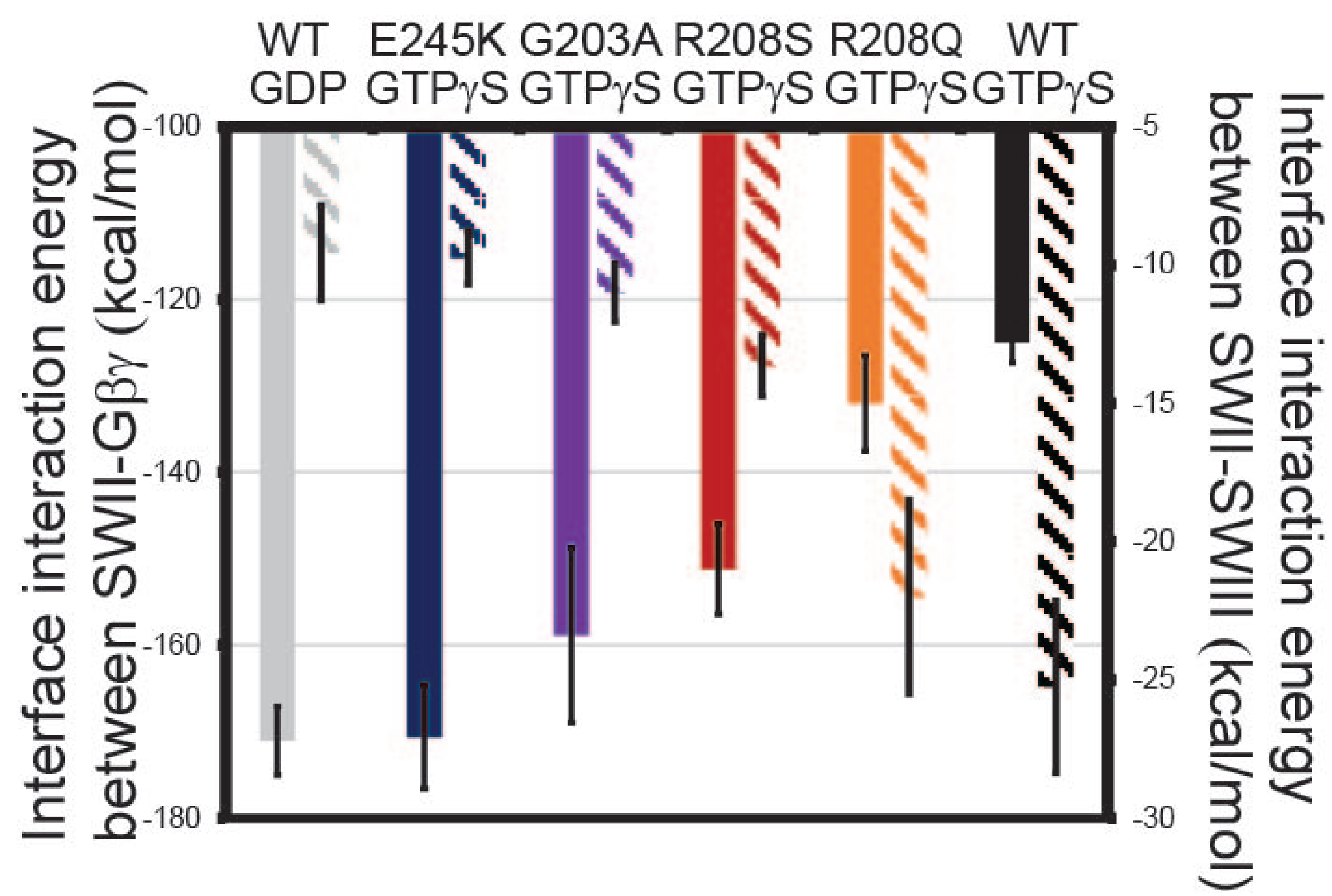

G $\alpha-G \beta \gamma$ Interface (GTP $\gamma S$ )

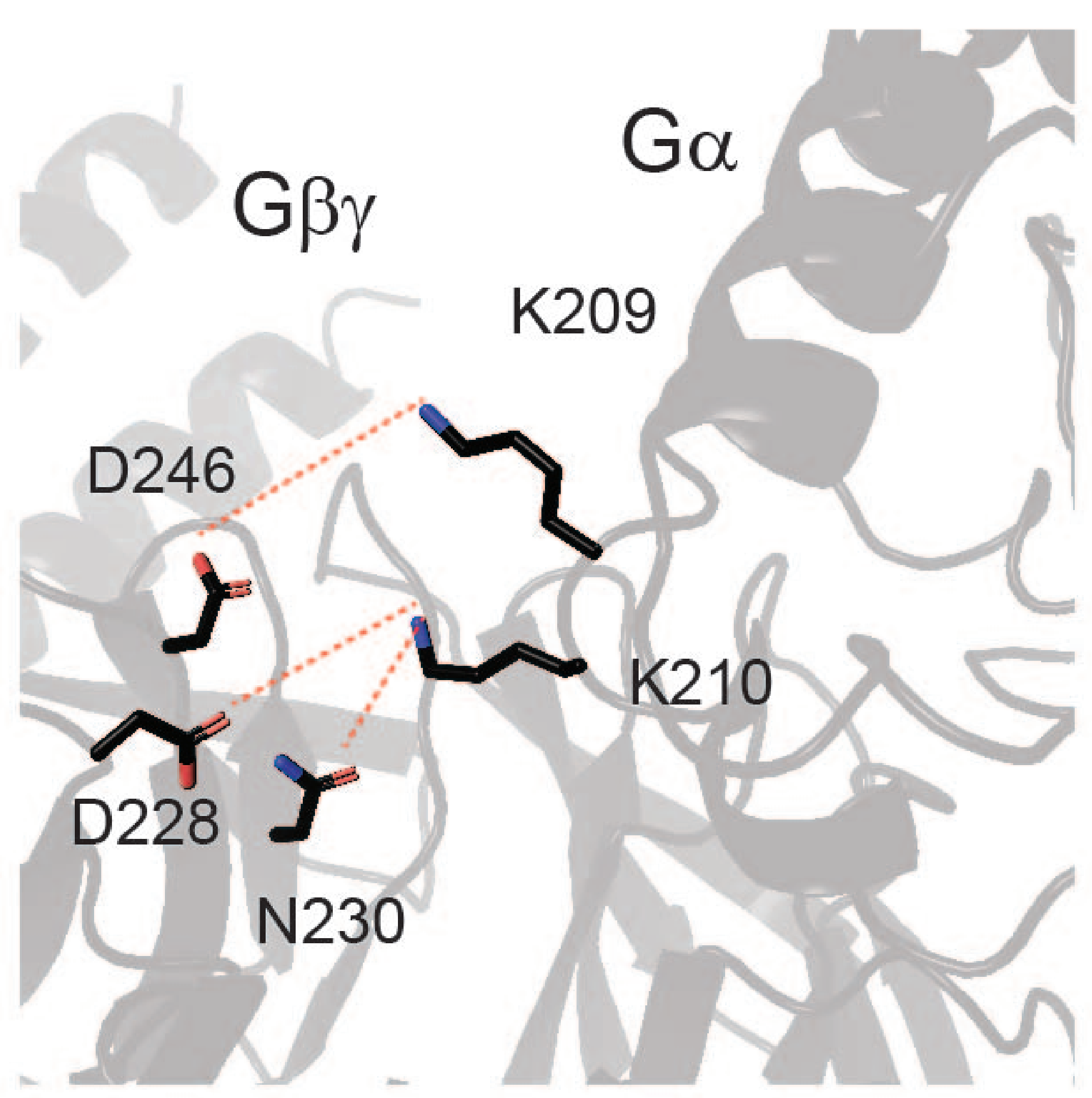

G $\alpha-G \beta \gamma$ Interface (GDP)

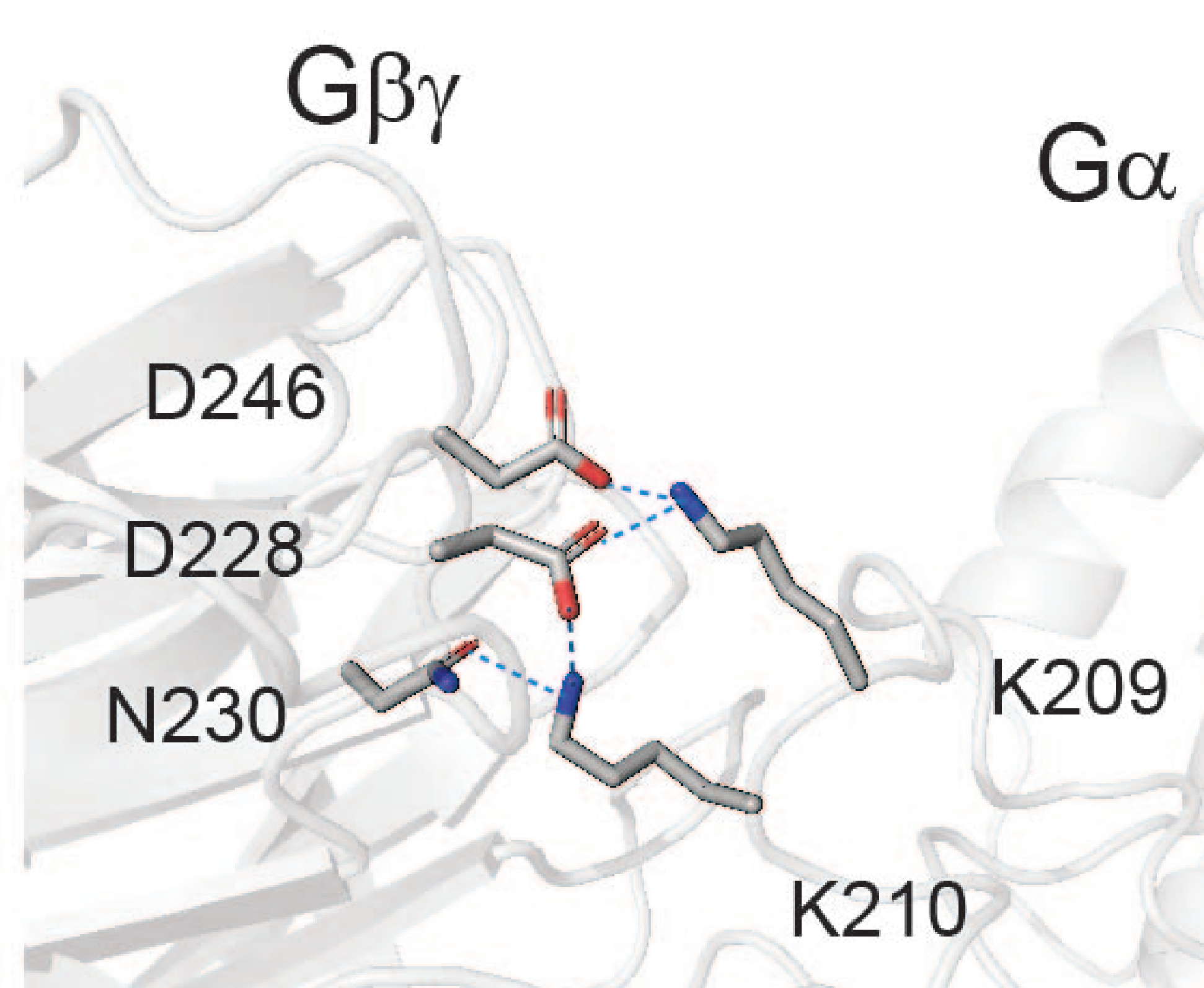


A
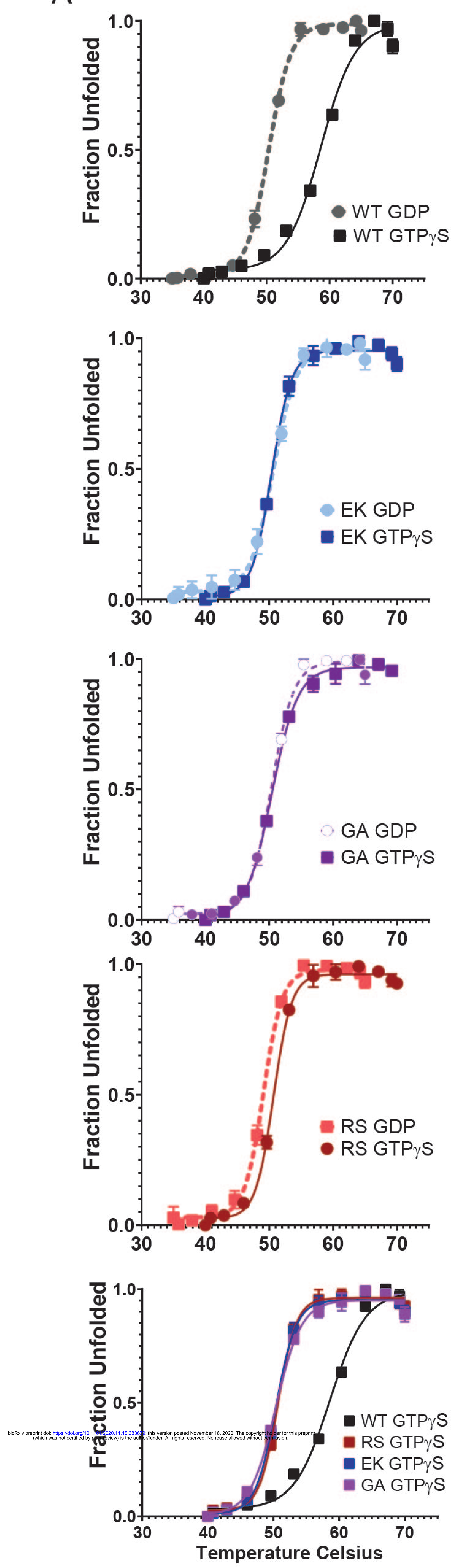

B

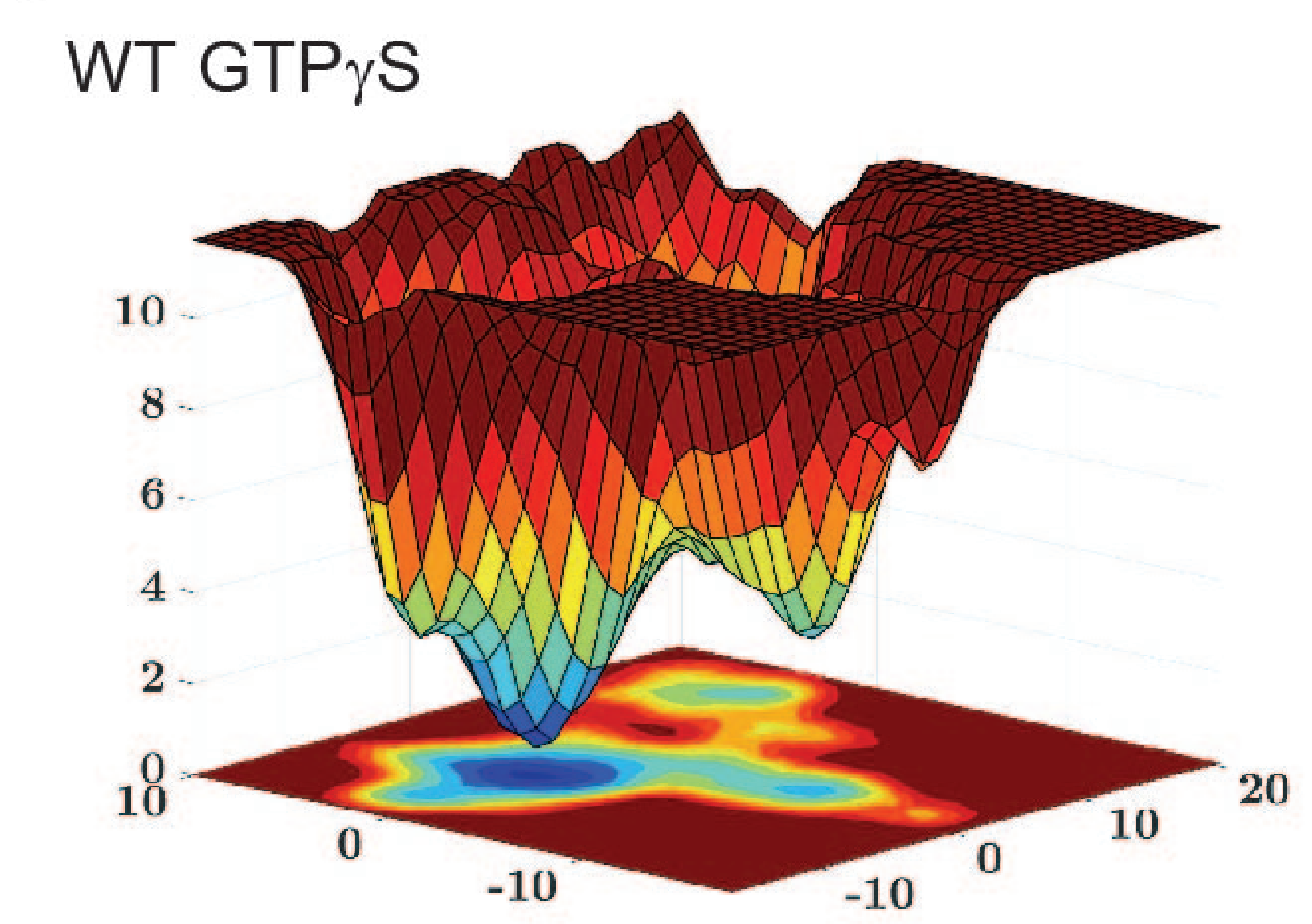

EK GTP $\gamma S$

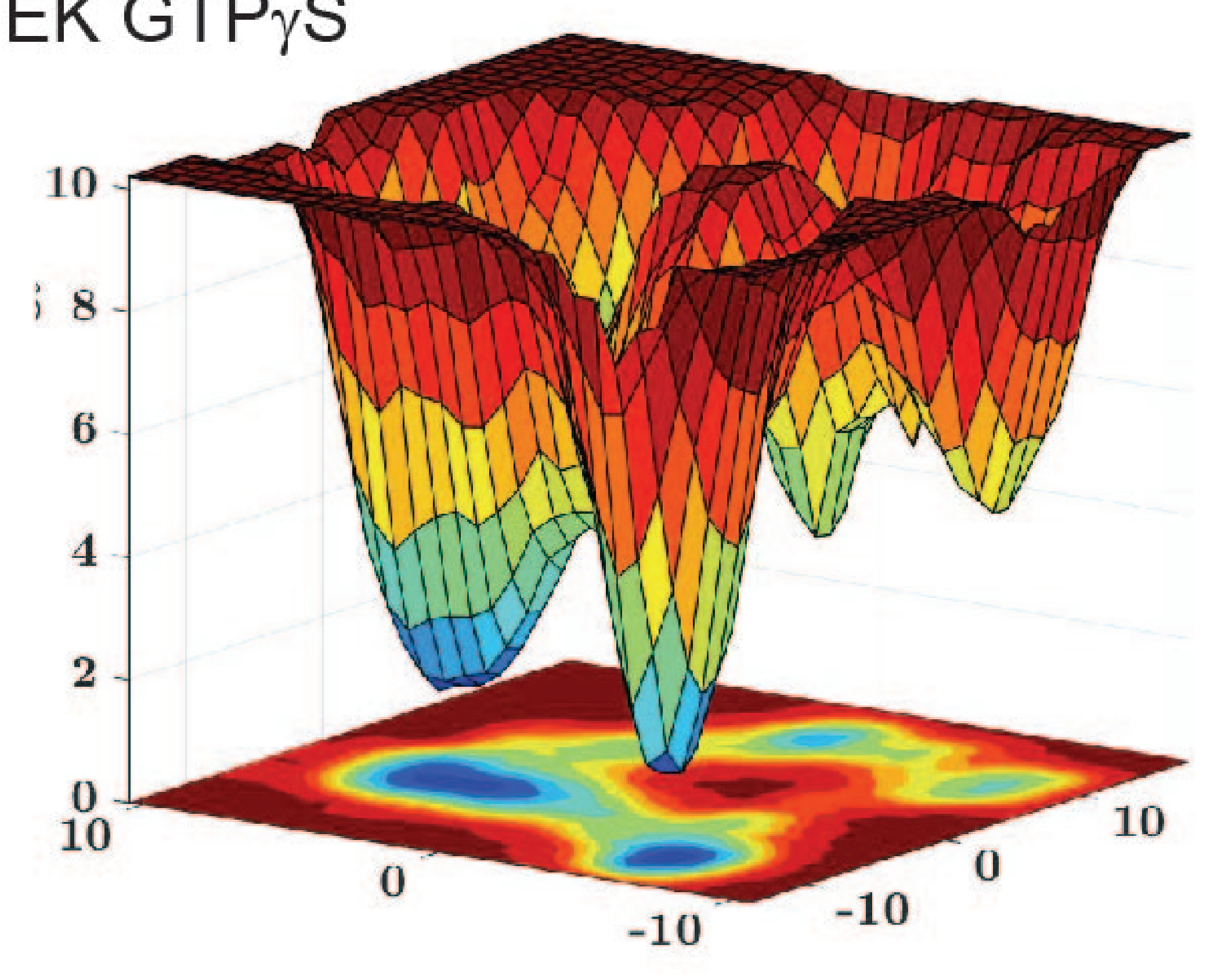

GA GTP $\gamma$ S

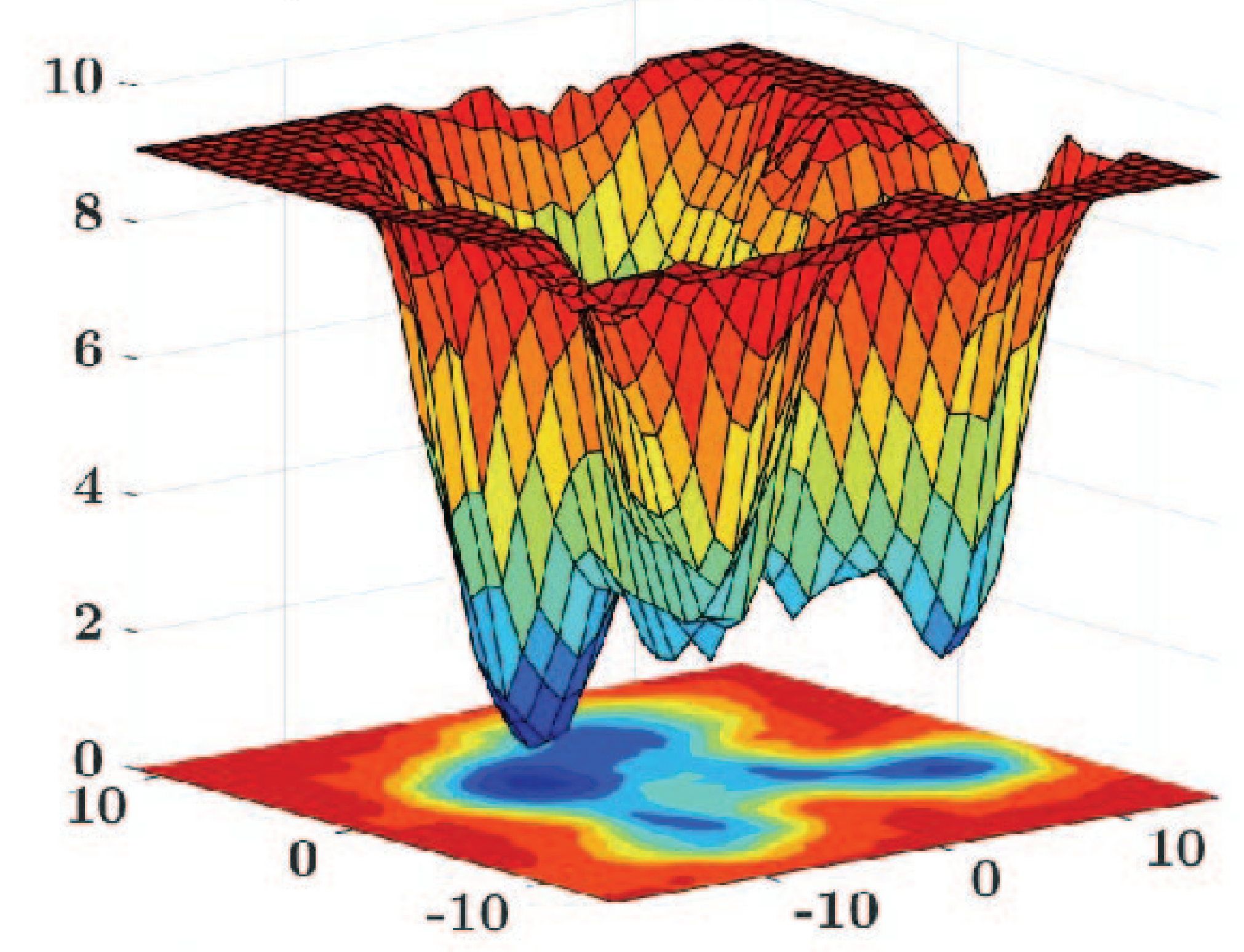

RS GTP $\gamma$ S

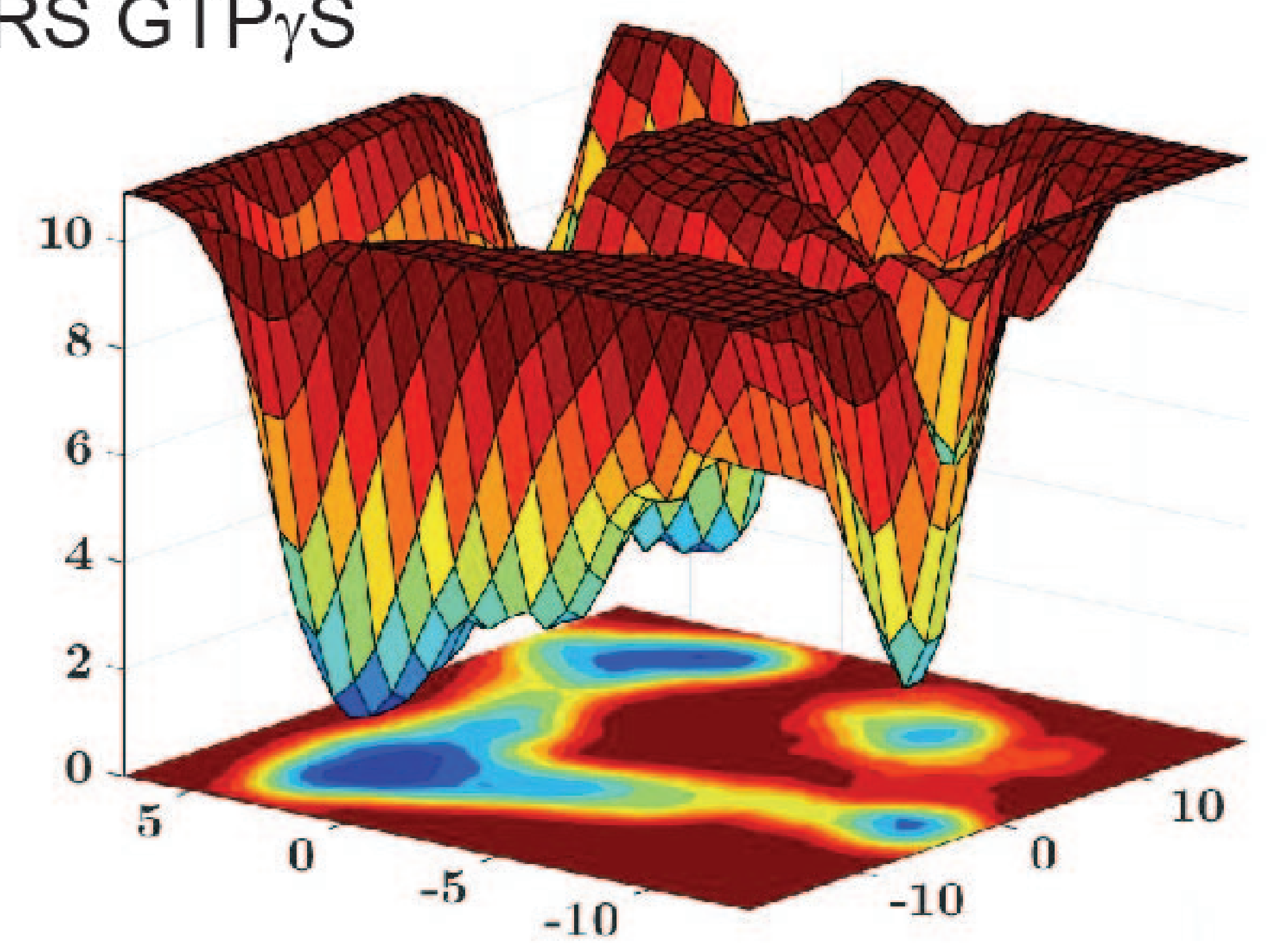

WT GDP

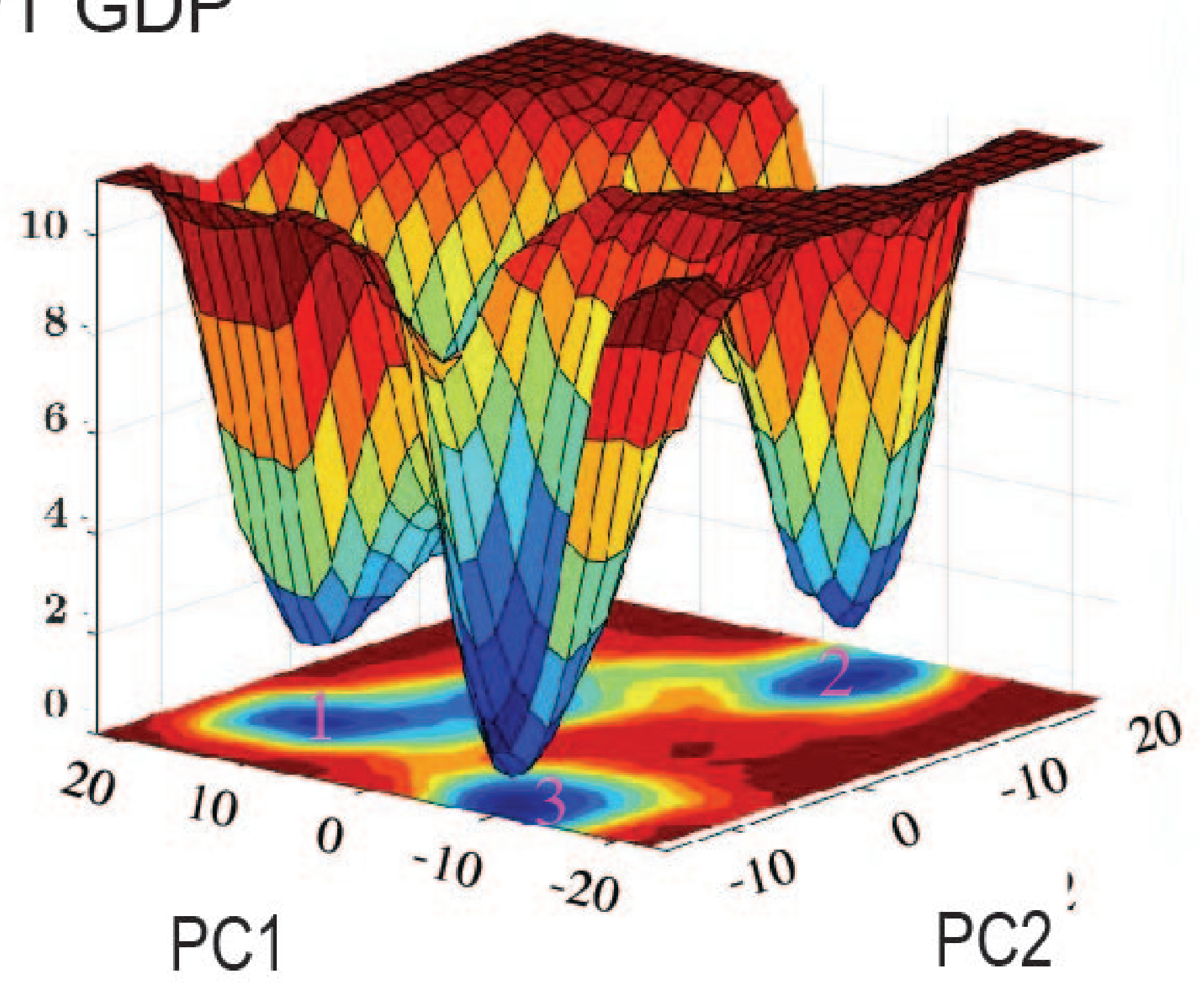

C
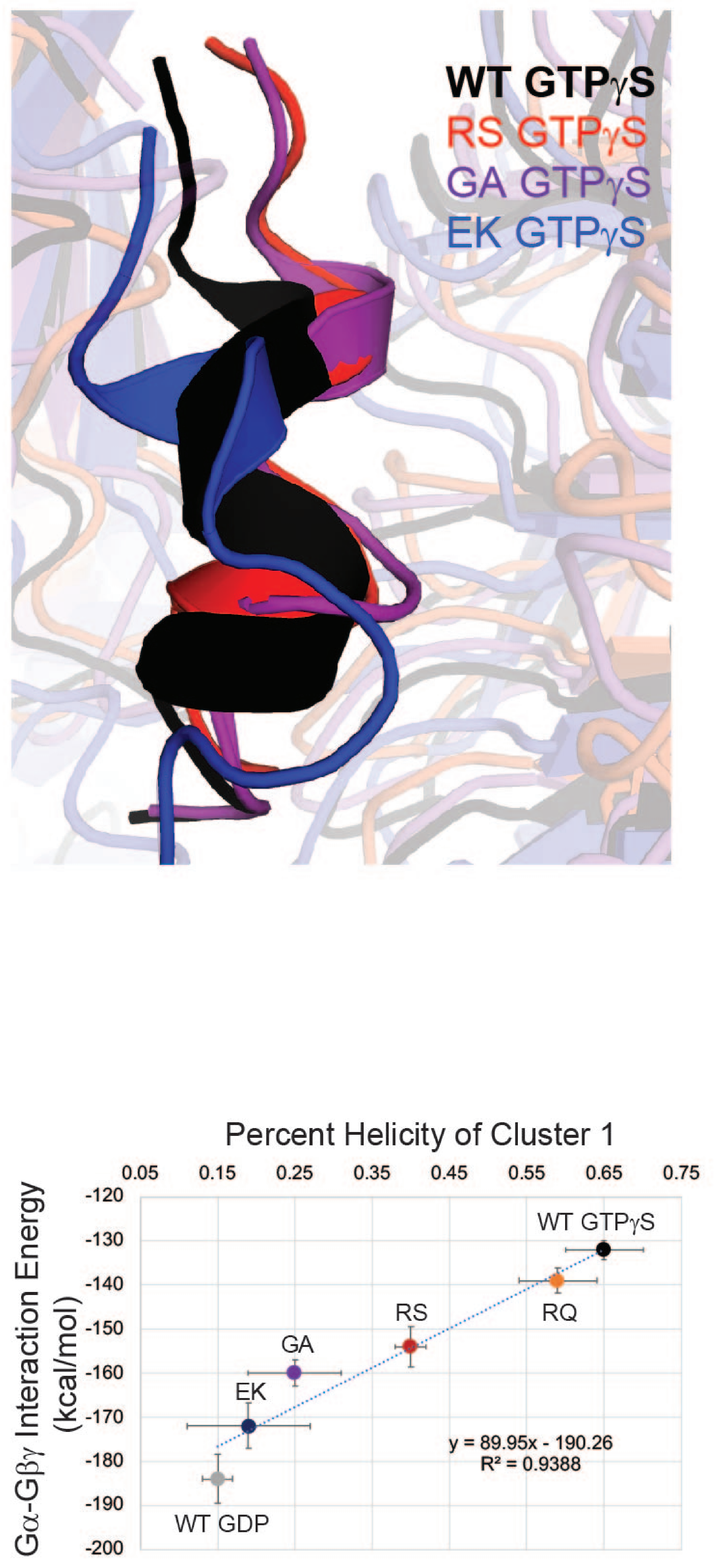

D

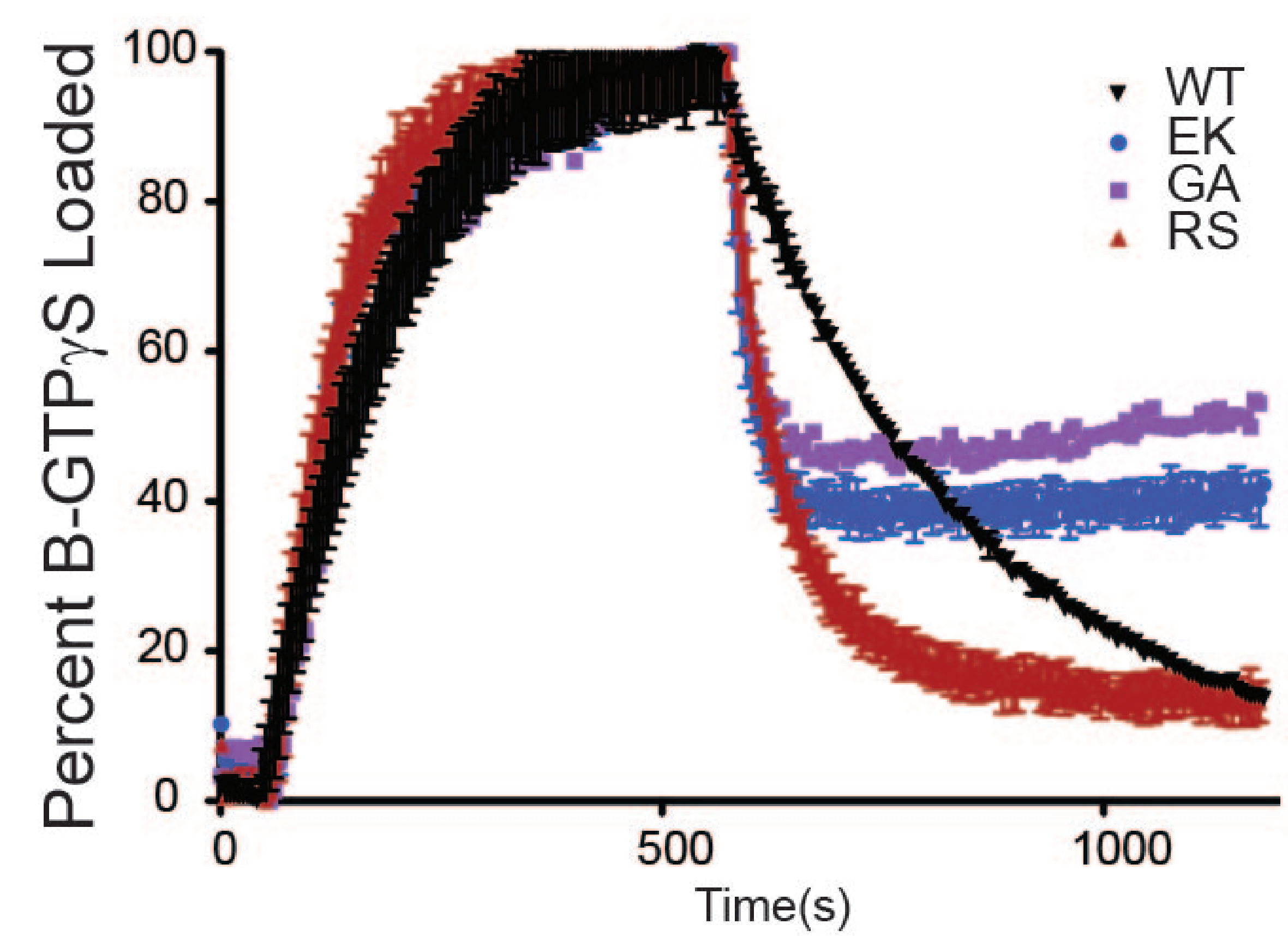


E

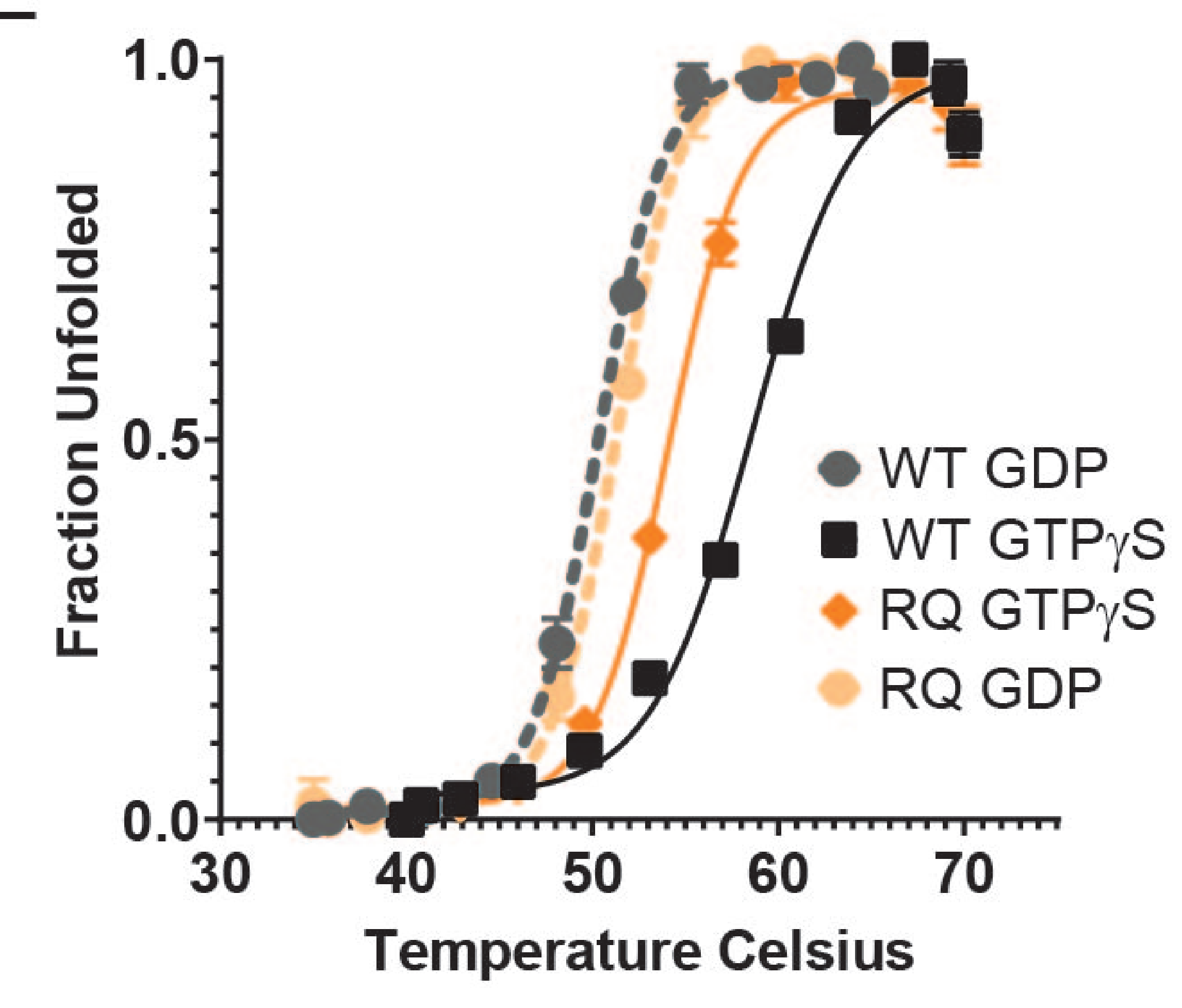

$\mathrm{H}$
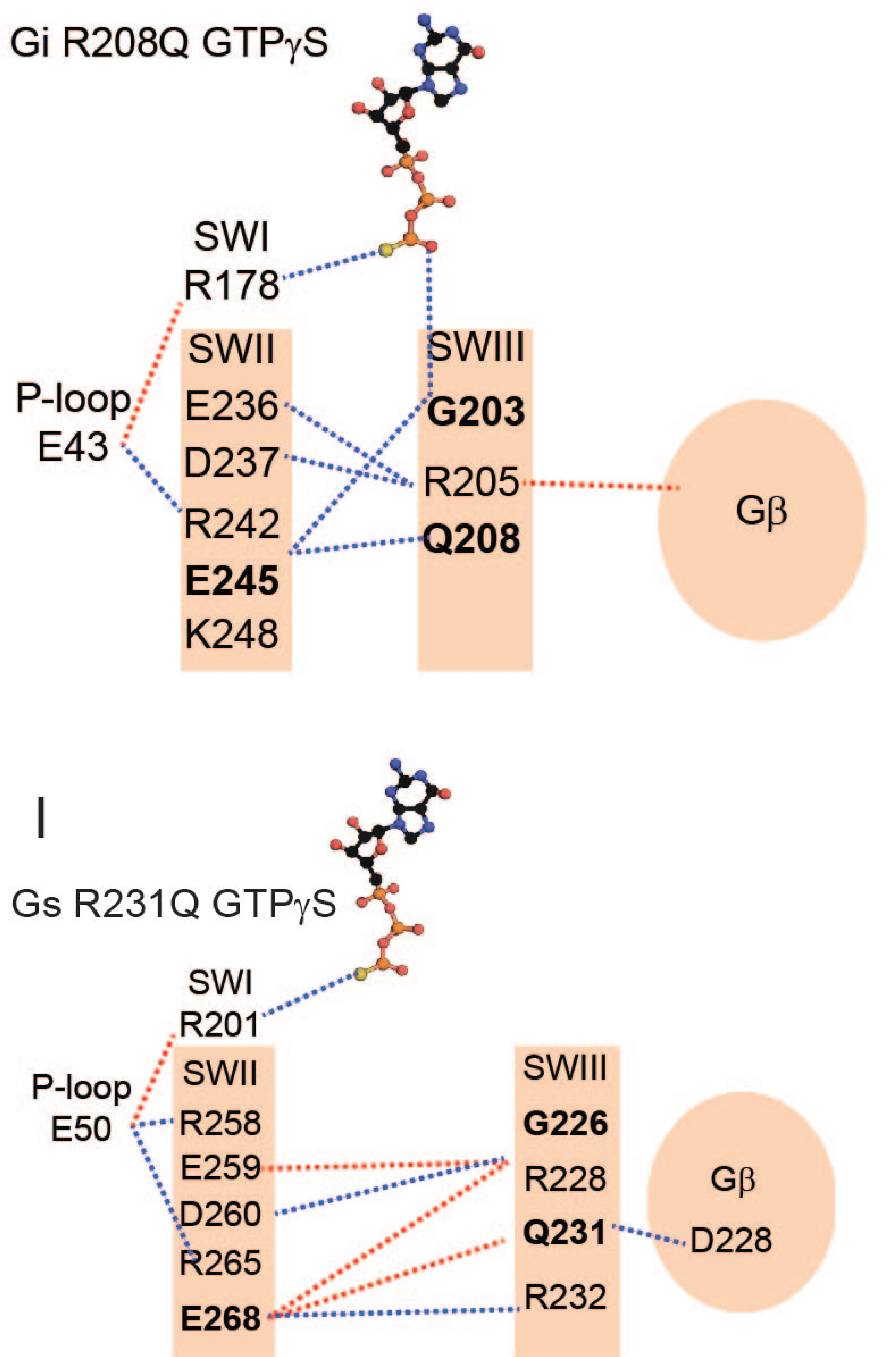

$\mathrm{F}$
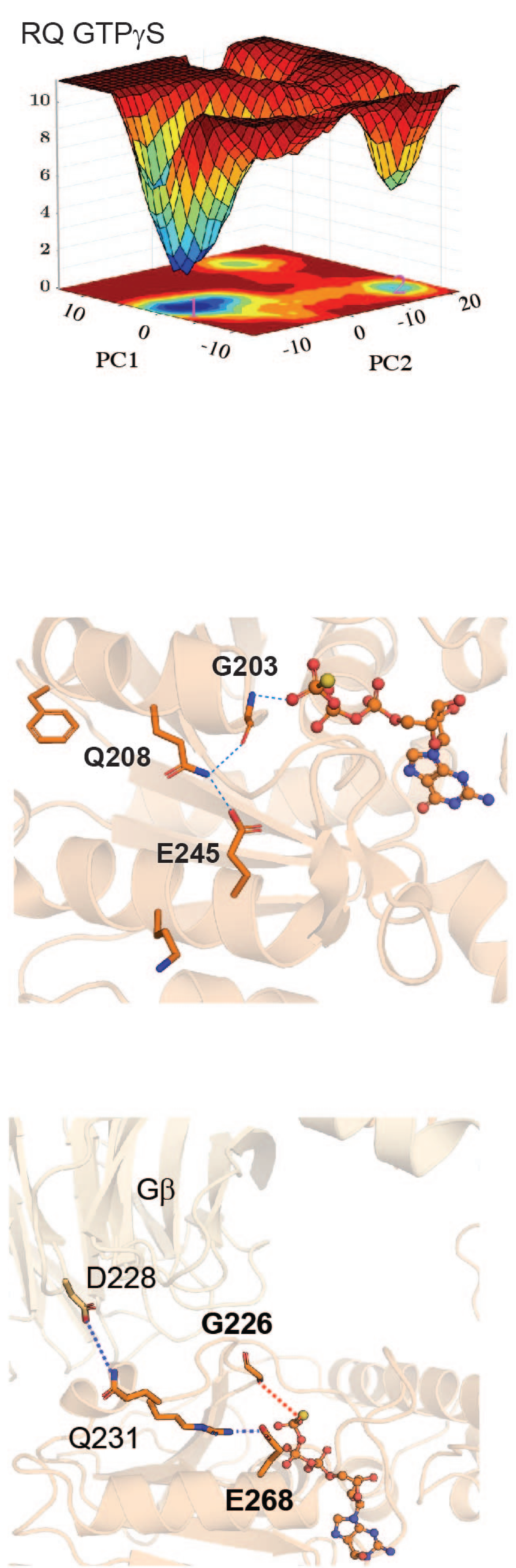

G
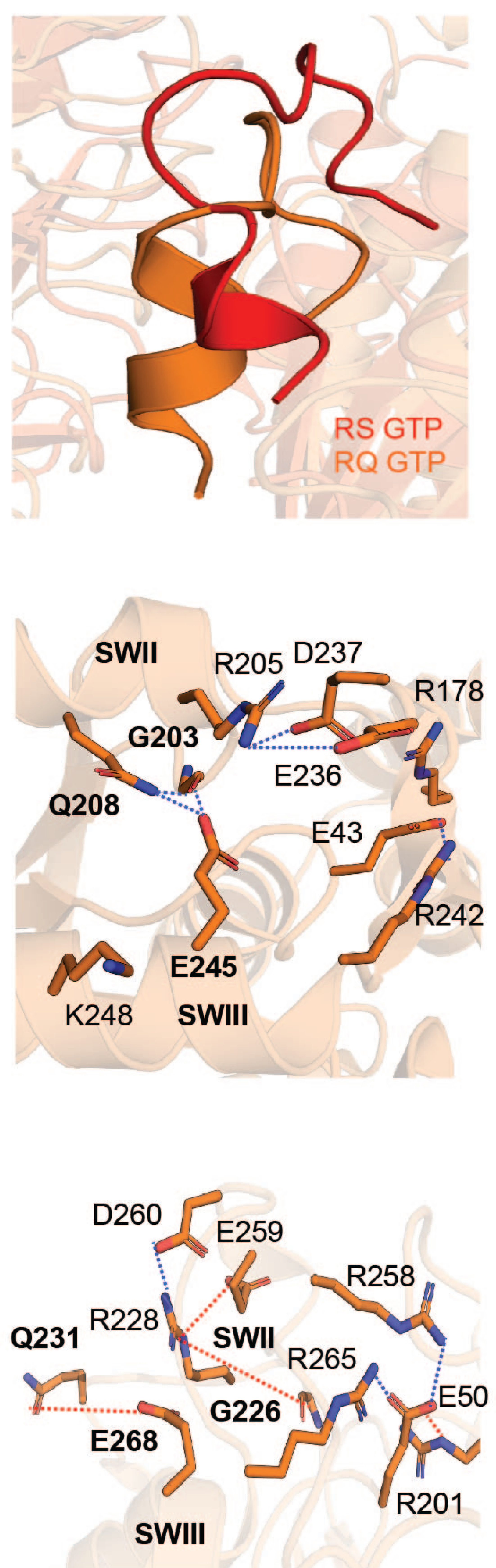

J

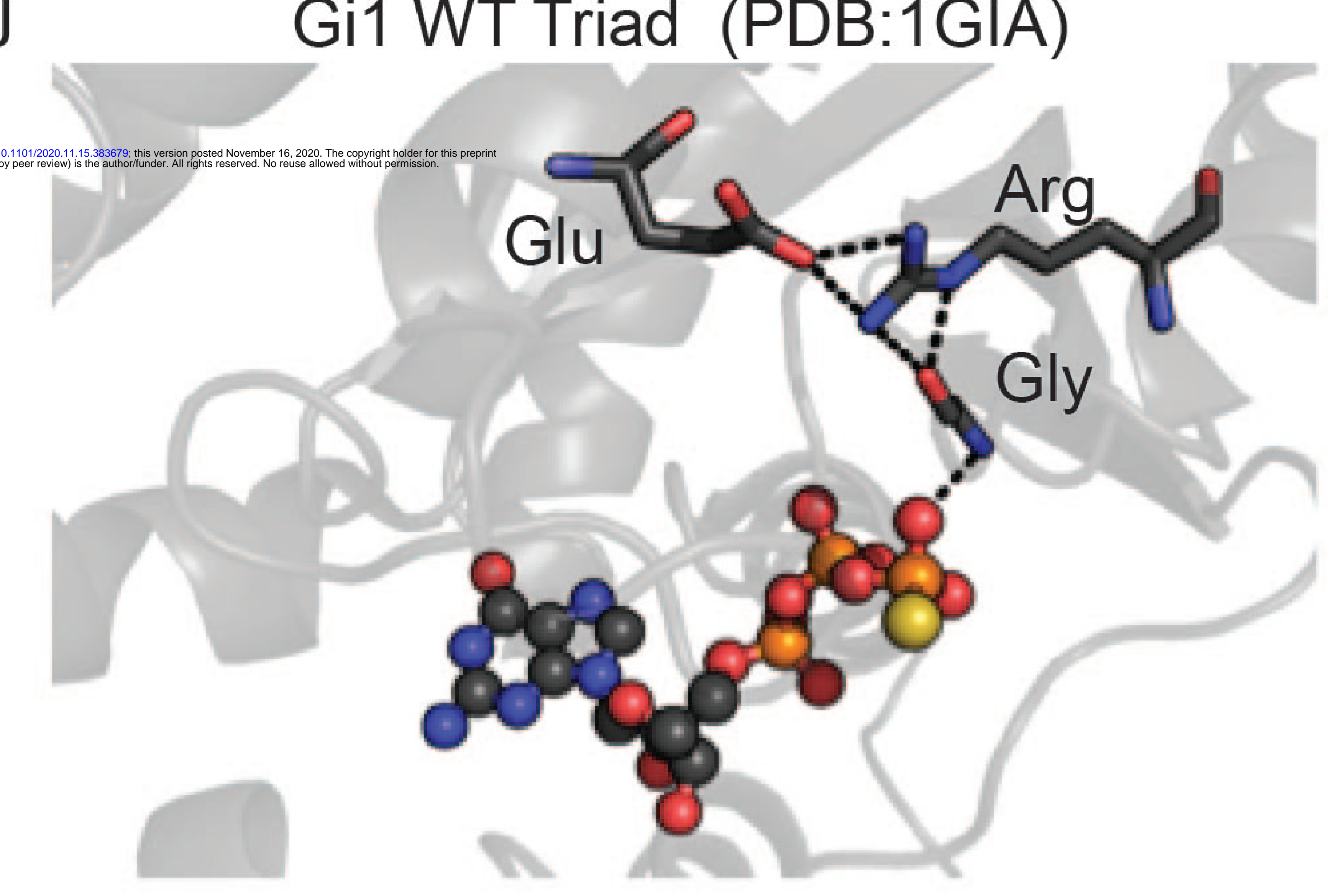

Gi1 RQ Triad (PDB:6M8H)

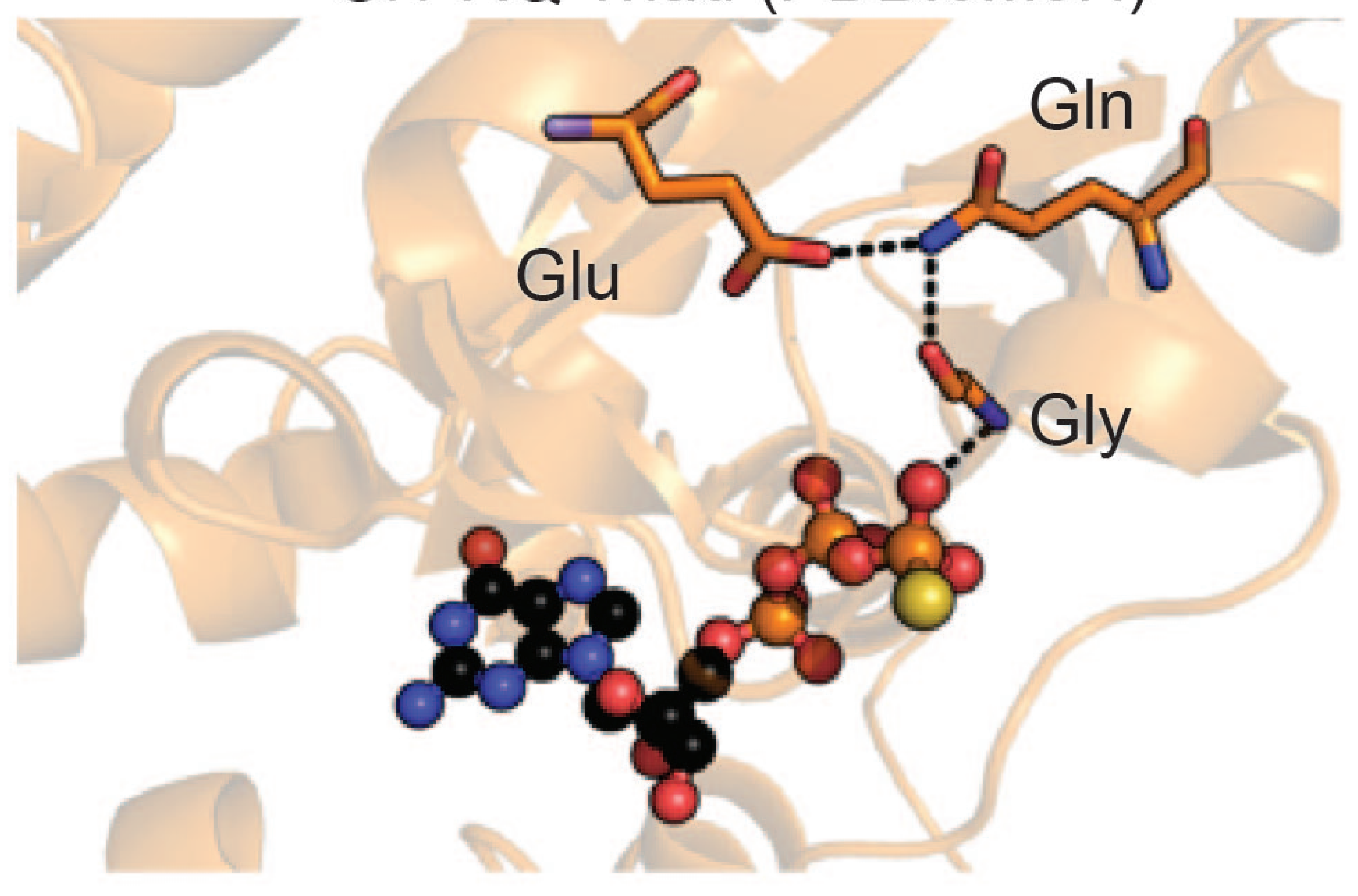



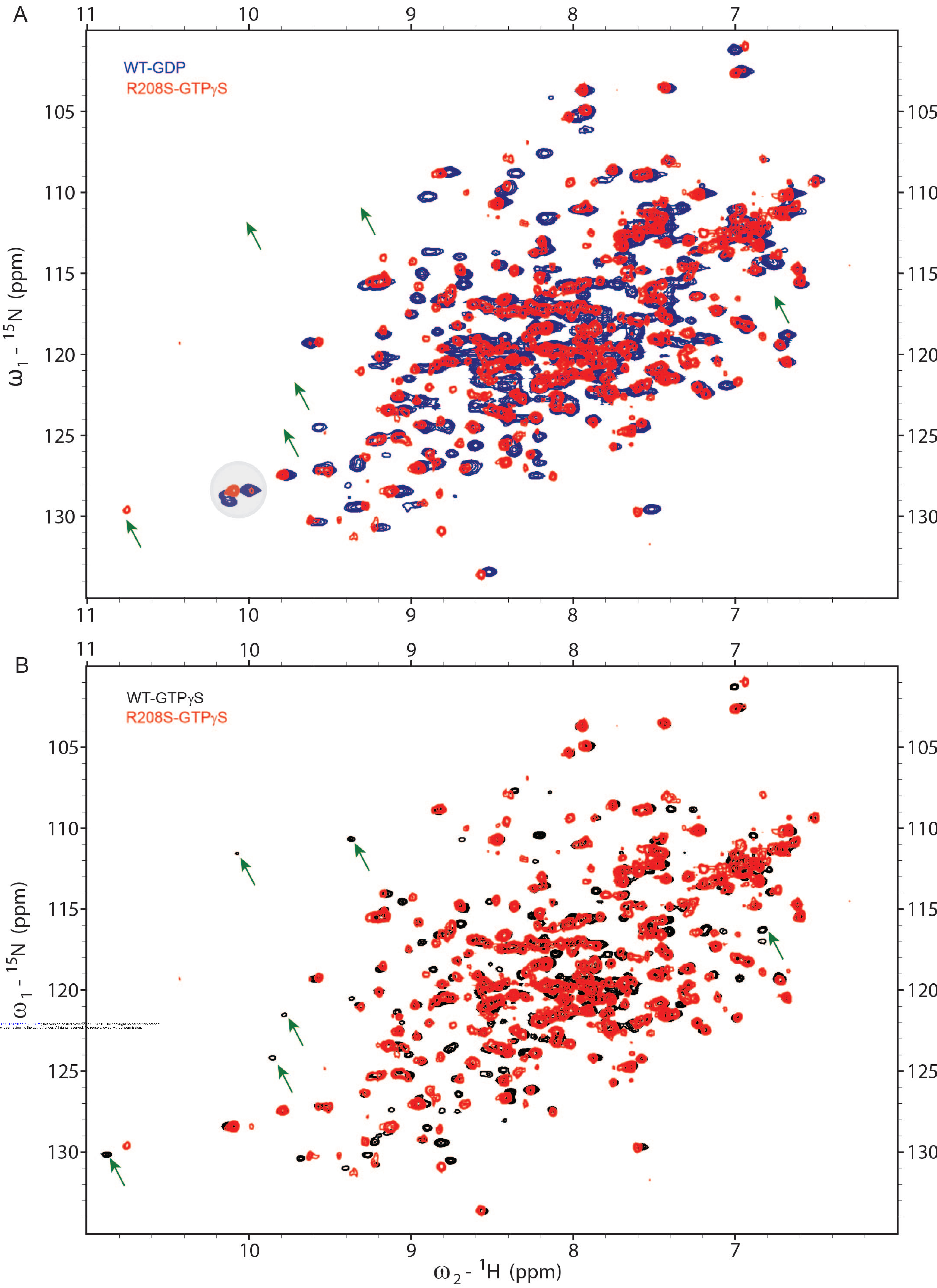


\section{Table S1}

\begin{tabular}{|l|rrrr|}
\hline \multicolumn{5}{|c|}{$\mathrm{k}_{\text {on }}$ M-1 s-1 } \\
\hline & WT GDP & WT AlF & RS GDP & RS AIF \\
\hline $1 \mu \mathrm{M}$ & 0.1739 & 0.1089 & 0.1835 & 0.2097 \\
$3 \mu \mathrm{M}$ & 0.3786 & 0.2191 & 0.3431 & 0.3555 \\
$10 \mu \mathrm{M}$ & 0.6042 & 0.4996 & 0.5821 & 0.5791 \\
\hline
\end{tabular}

\begin{tabular}{|l|rr|rr|}
\hline \multicolumn{5}{|c|}{ Standard Error in $\mathrm{k}_{\text {on }}$} \\
\hline & \multicolumn{1}{|c|}{ WT GDP } & \multicolumn{1}{|c|}{ WT AlF } & \multicolumn{1}{|c|}{ RS GDP } & \multicolumn{1}{c|}{ RS AlF } \\
\hline $1 \mu \mathrm{M}$ & 0.006226 & 0.00811 & 0.005236 & 0.005273 \\
$3 \mu \mathrm{M}$ & 0.008694 & 0.005218 & 0.007435 & 0.008023 \\
$10 \mu \mathrm{M}$ & 0.01724 & 0.01252 & 0.01729 & 0.01692 \\
\hline
\end{tabular}

\begin{tabular}{|l|rrrr|}
\hline \multicolumn{5}{|c|}{$\mathrm{k}_{\text {off }} \mathrm{s}-1$} \\
\hline & WT GDP & WT AlF & \multicolumn{1}{c|}{ RS GDP } & RS AlF \\
\hline $1 \mu \mathrm{M}$ & 0.1542 & 0.1188 & 0.1532 & 0.1401 \\
$3 \mu \mathrm{M}$ & 0.2295 & 0.1639 & 0.1634 & 0.1644 \\
$10 \mu \mathrm{M}$ & 0.2626 & 0.1922 & 0.186 & 0.1786 \\
\hline
\end{tabular}

\begin{tabular}{|l|rrrr|}
\hline \multicolumn{5}{|c|}{ B max } \\
\hline & WT GDP & WT AIF & RS GDP & RS AIF \\
\hline $1 \mu \mathrm{M}$ & 0.1739 & 0.1141 & 0.208 & 0.2525 \\
$3 \mu \mathrm{M}$ & 0.4217 & 0.3171 & 0.4746 & 0.5472 \\
$10 \mu \mathrm{M}$ & 0.6234 & 0.6609 & 0.6668 & 0.6999 \\
\hline
\end{tabular}


G11 $208 \quad 210 \quad 21:\left[\begin{array}{lllllllllllllllllll}214 & 216 & 218 & 220 & 222 & 224 & 226 & 228 & 230 & 232 & 234 & 236 & 238 & 240 & 242 & 244 & 246 & 248 & 250\end{array}\right.$ \begin{tabular}{llllllllllllllllllllllllllll}
\hline & \\
\hline
\end{tabular}

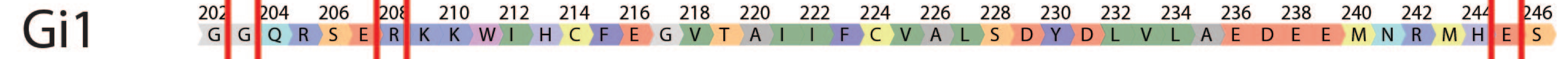

\begin{tabular}{llllllllllllllllllllll|l}
210 & 212 & 214 & 21 & 218 & 220 & 222 & 224 & 226 & 228 & 230 & 232 & 234 & 236 & 238 & 240 & 242 & 244 & 246 & 248 & 250 & 252 & 254
\end{tabular}

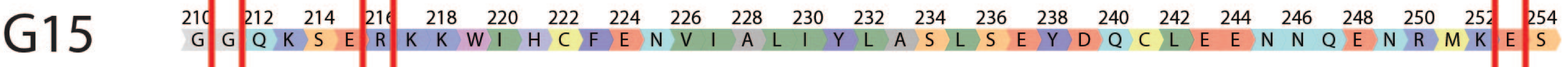

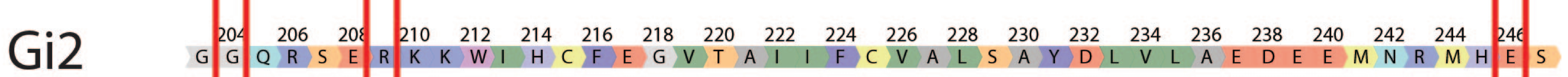

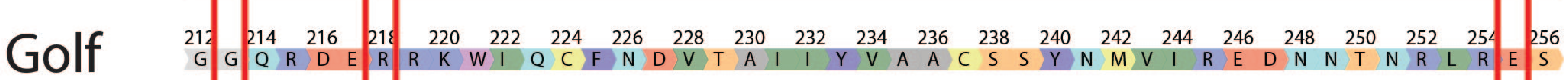

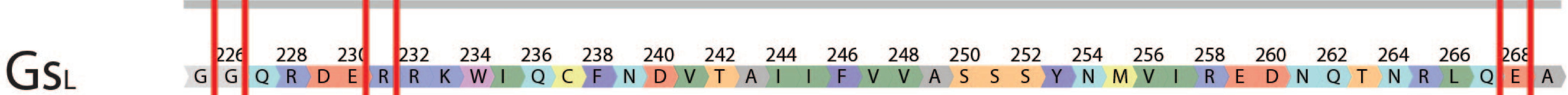

GOB $\quad 204 \quad 206 \quad 208 \quad \begin{array}{lllllllllllllllllll}210 & 212 & 214 & 216 & 218 & 220 & 222 & 224 & 226 & 228 & 230 & 232 & 234 & 236 & 238 & 240 & 242 & 244 & 246\end{array}$

G G G Q R S E R K K WI HCF E D V T A I I F C V A L S G Y D Q V L HE DETTNRMHES

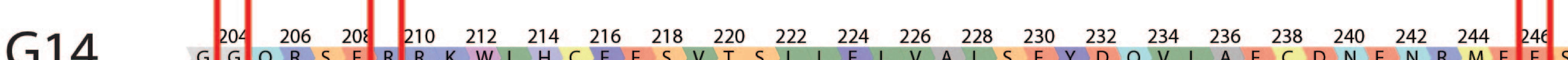

G14 G G Q R S E R R R W W

GSs $\quad G \quad$\begin{tabular}{lllllllllllllllllllllllllll}
212 & 214 & 216 & 218 & 220 & 222 & 224 & 226 & 228 & 230 & 232 & 234 & 236 & 238 & 240 & 242 & 244 & 246 & 248 & 250 & 252 & 25 & \\
\hline
\end{tabular}

$\begin{array}{llllllllllllllllllllll}204 & 206 & 208 & 210 & 212 & 214 & 216 & 218 & 220 & 222 & 224 & 226 & 228 & 230 & 232 & 234 & 236 & 238 & 240 & 242 & 244 & 246\end{array}$

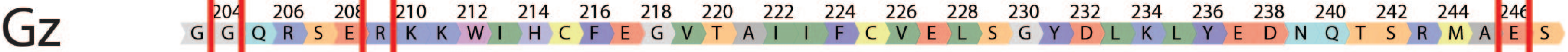

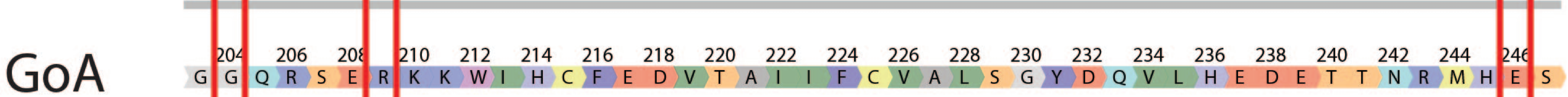

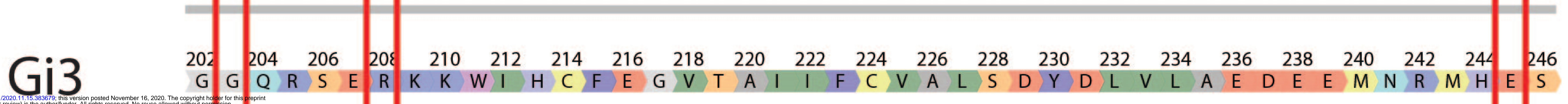

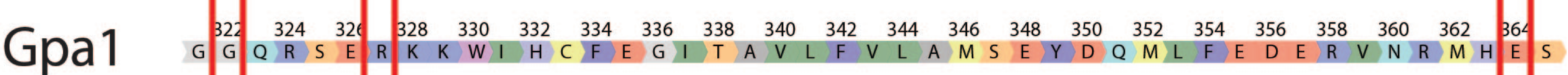

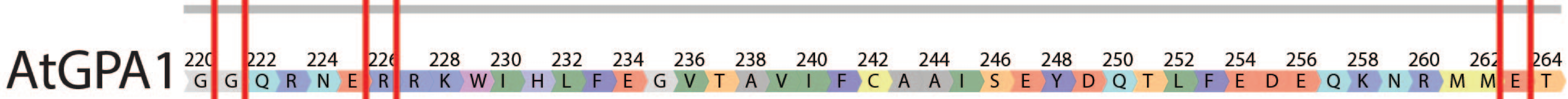

Gq $\quad$ G 
Gas (1AZT)

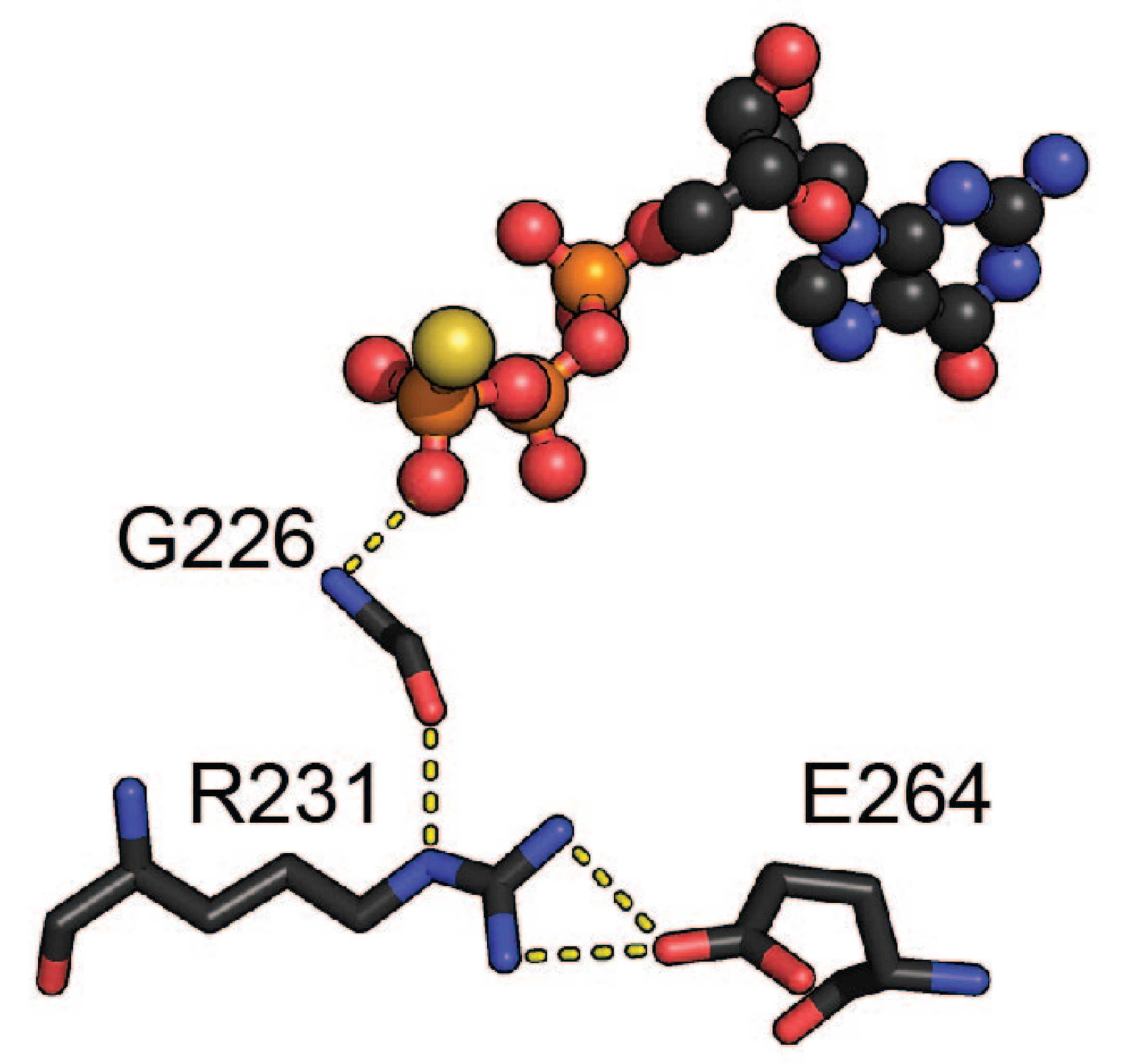

Gat (1TND)

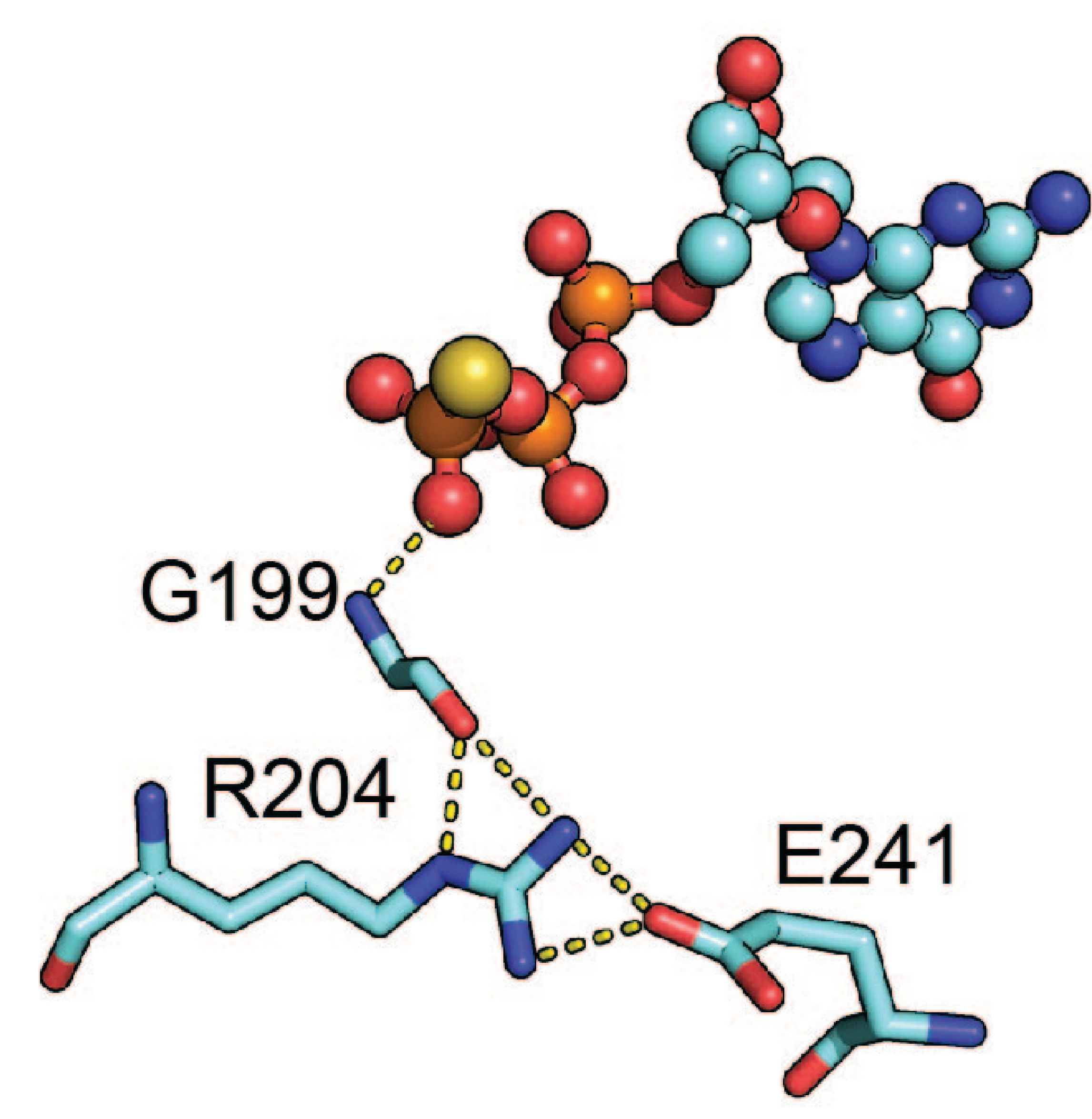

$\mathrm{G} \alpha \mathrm{q}(3 \mathrm{OHM})$

${ }^{*} \mathrm{AlF}_{4}$

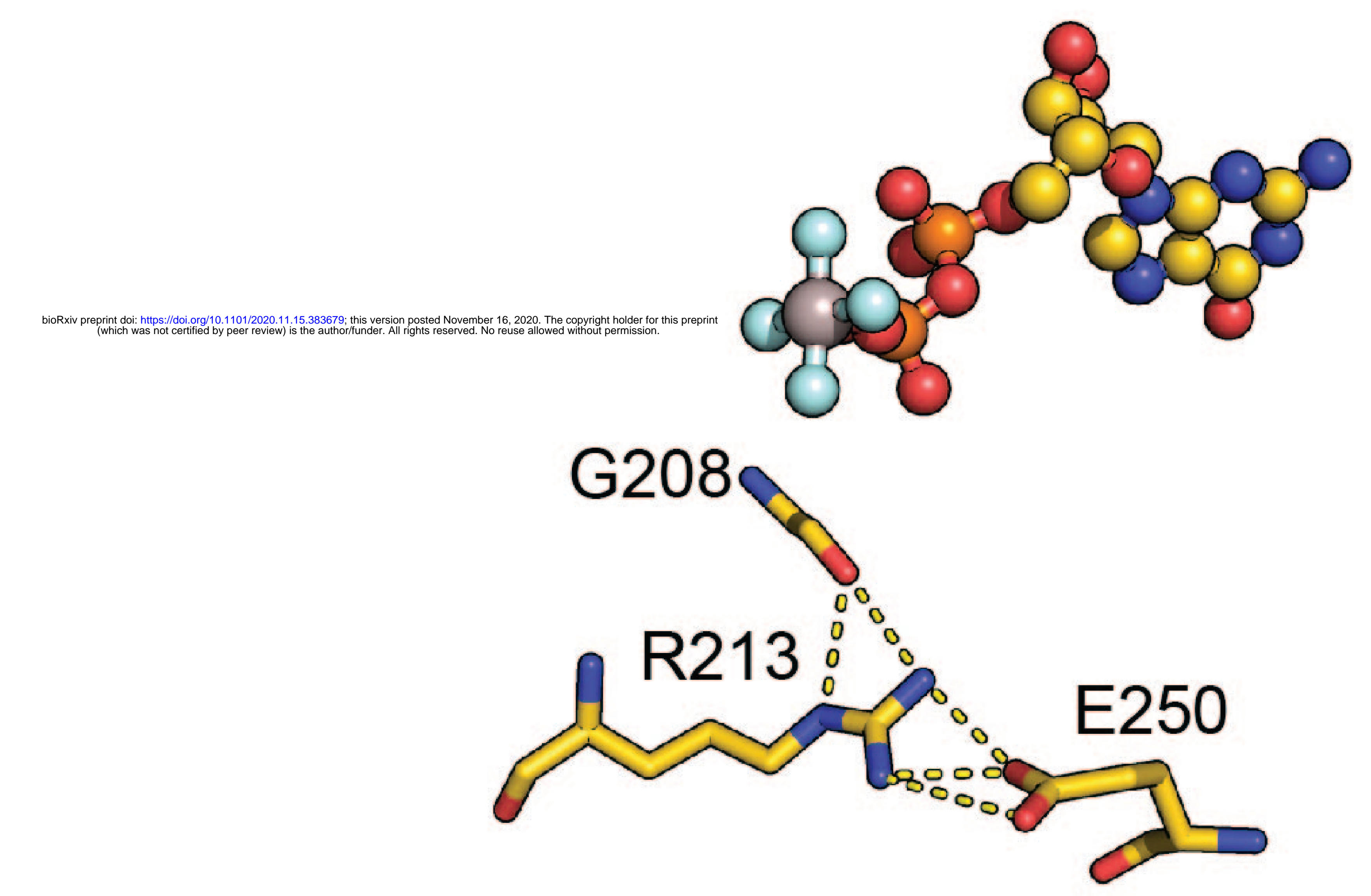

Gai1 (1GIA)

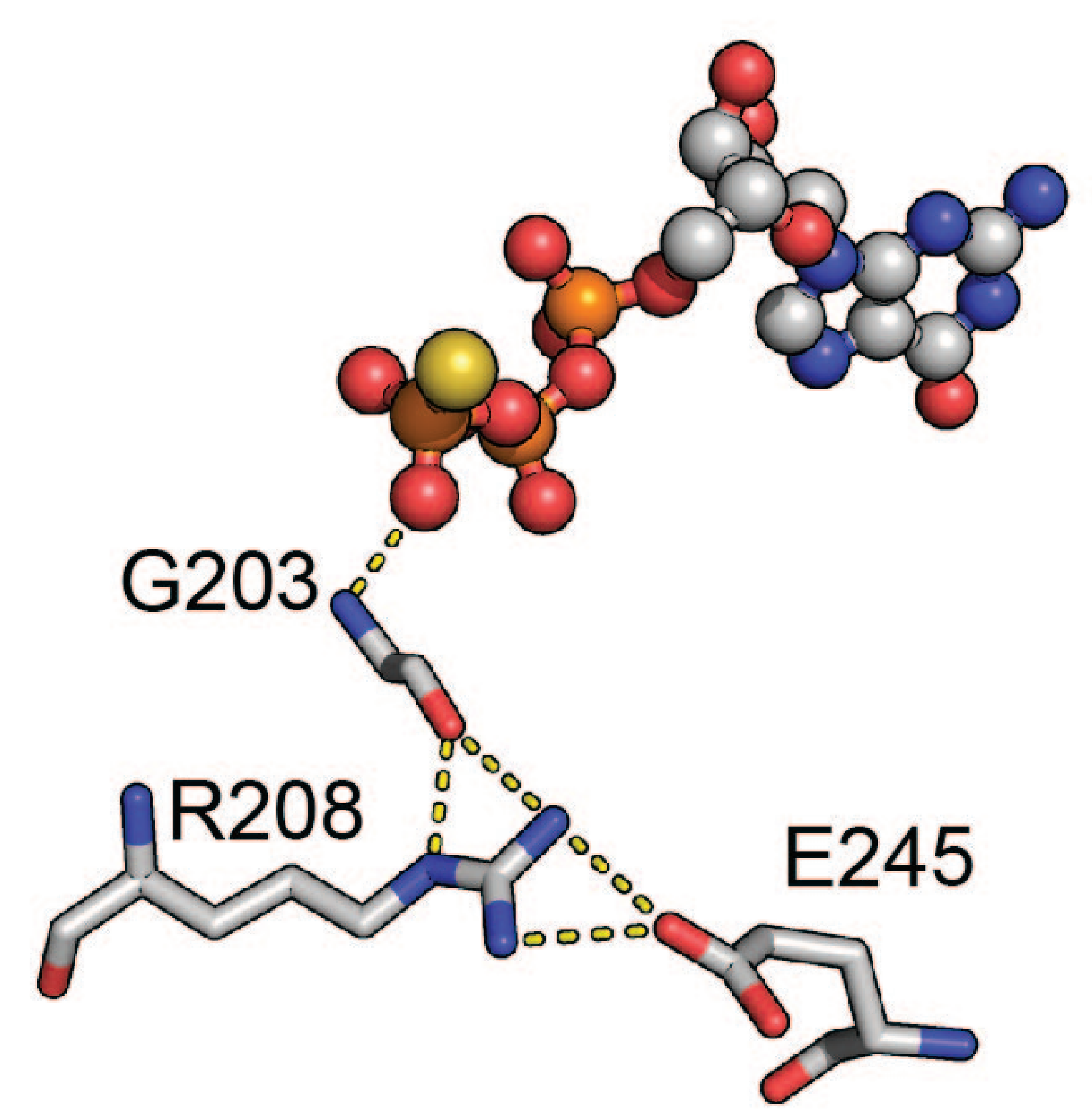

$\mathrm{G} \alpha 13$ (3CX8)

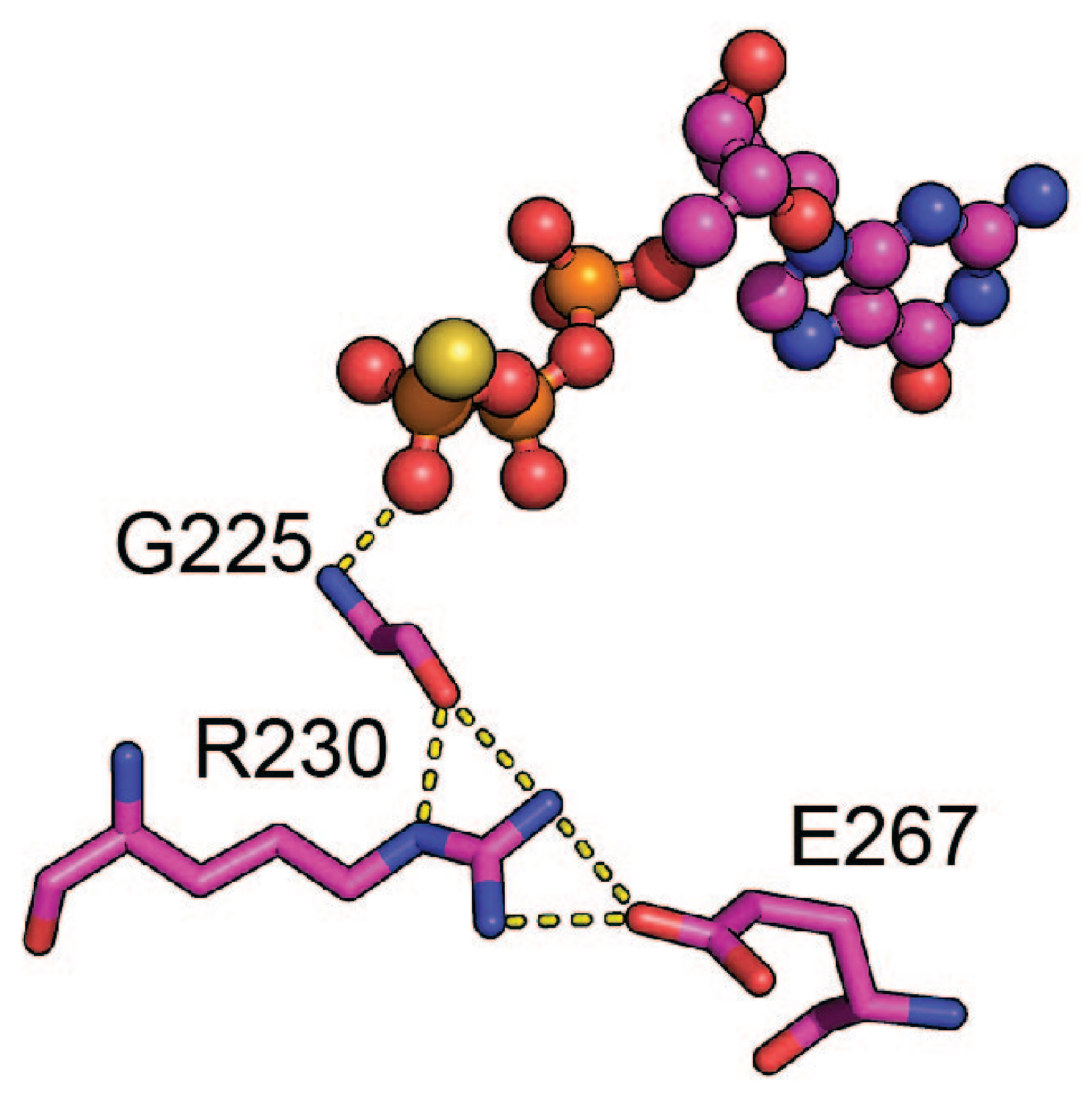

AtGPA1 (2XTZ)

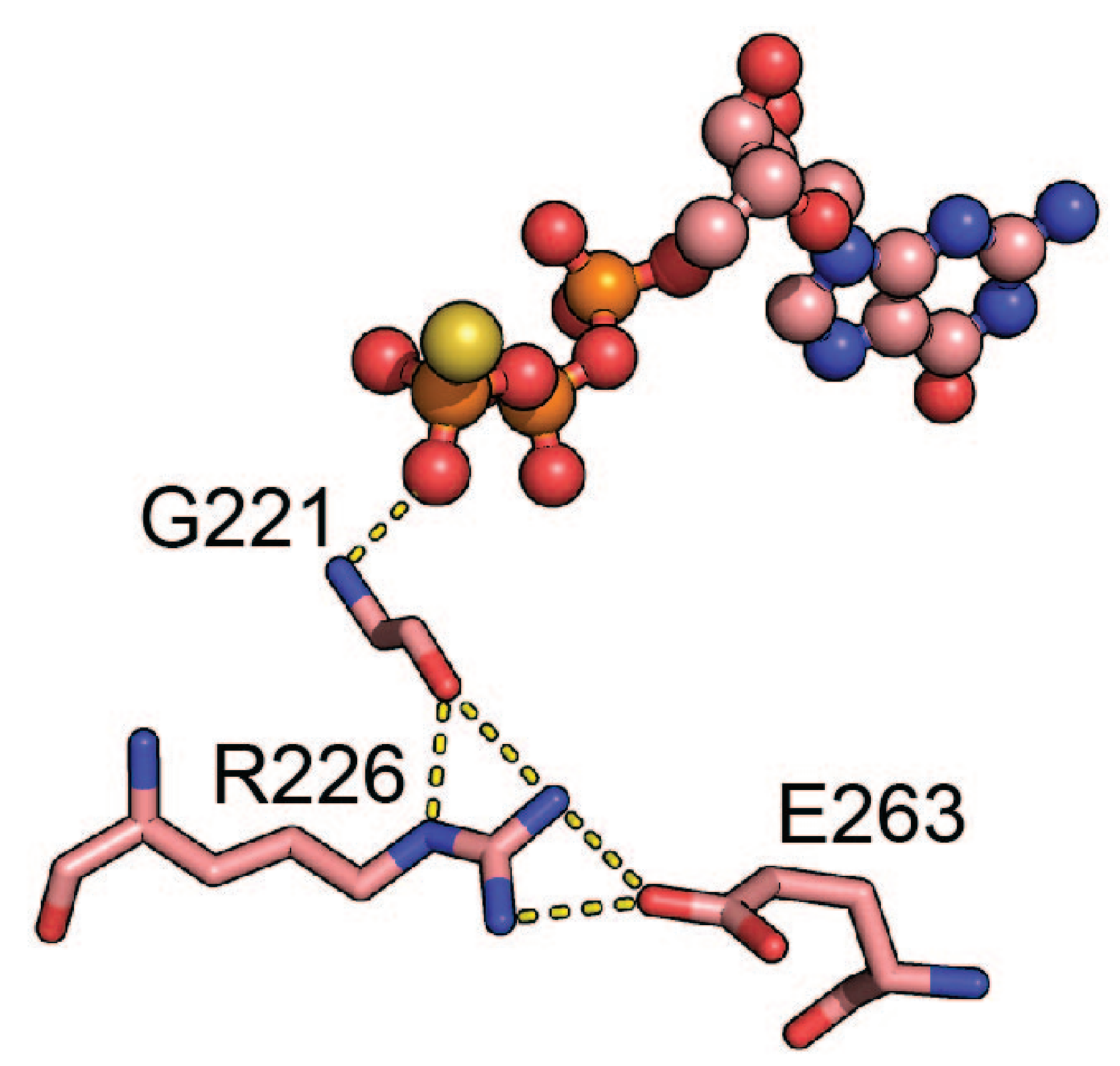



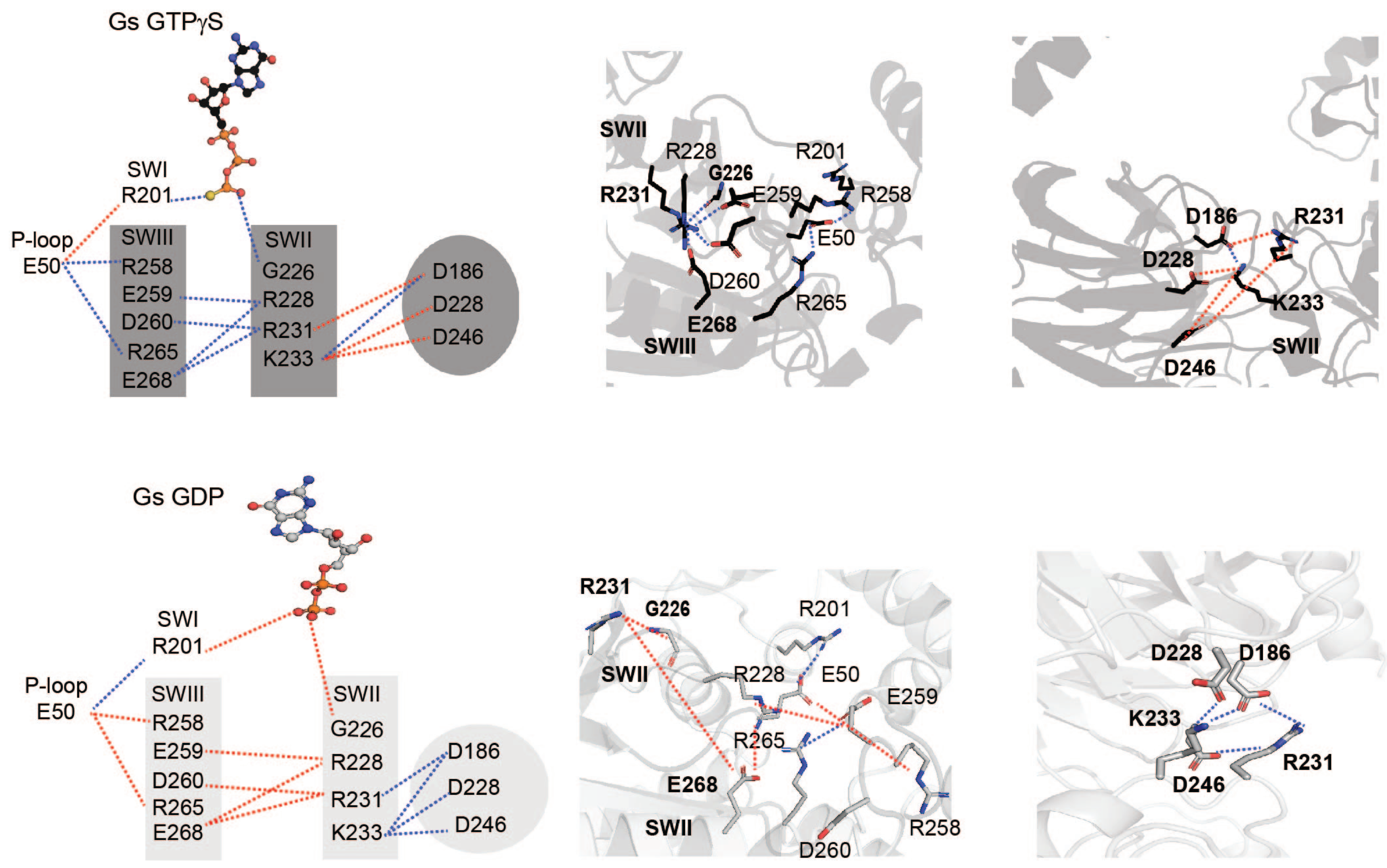

B
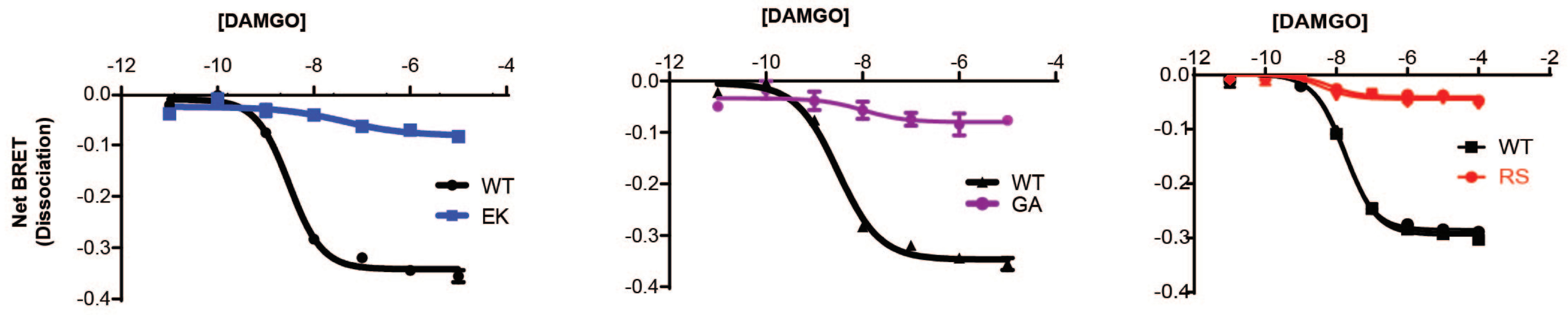


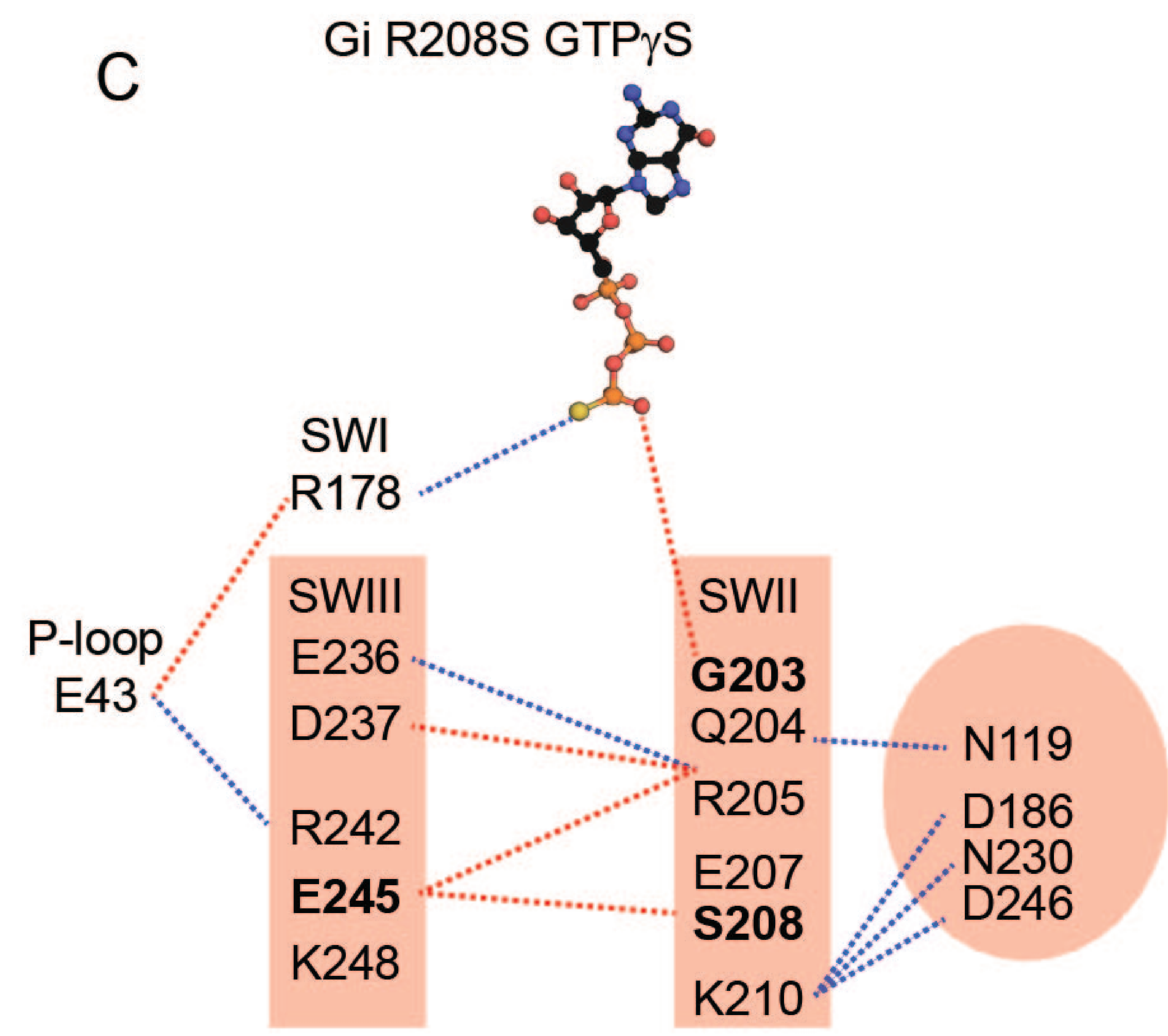

SWII-SWIII Interface
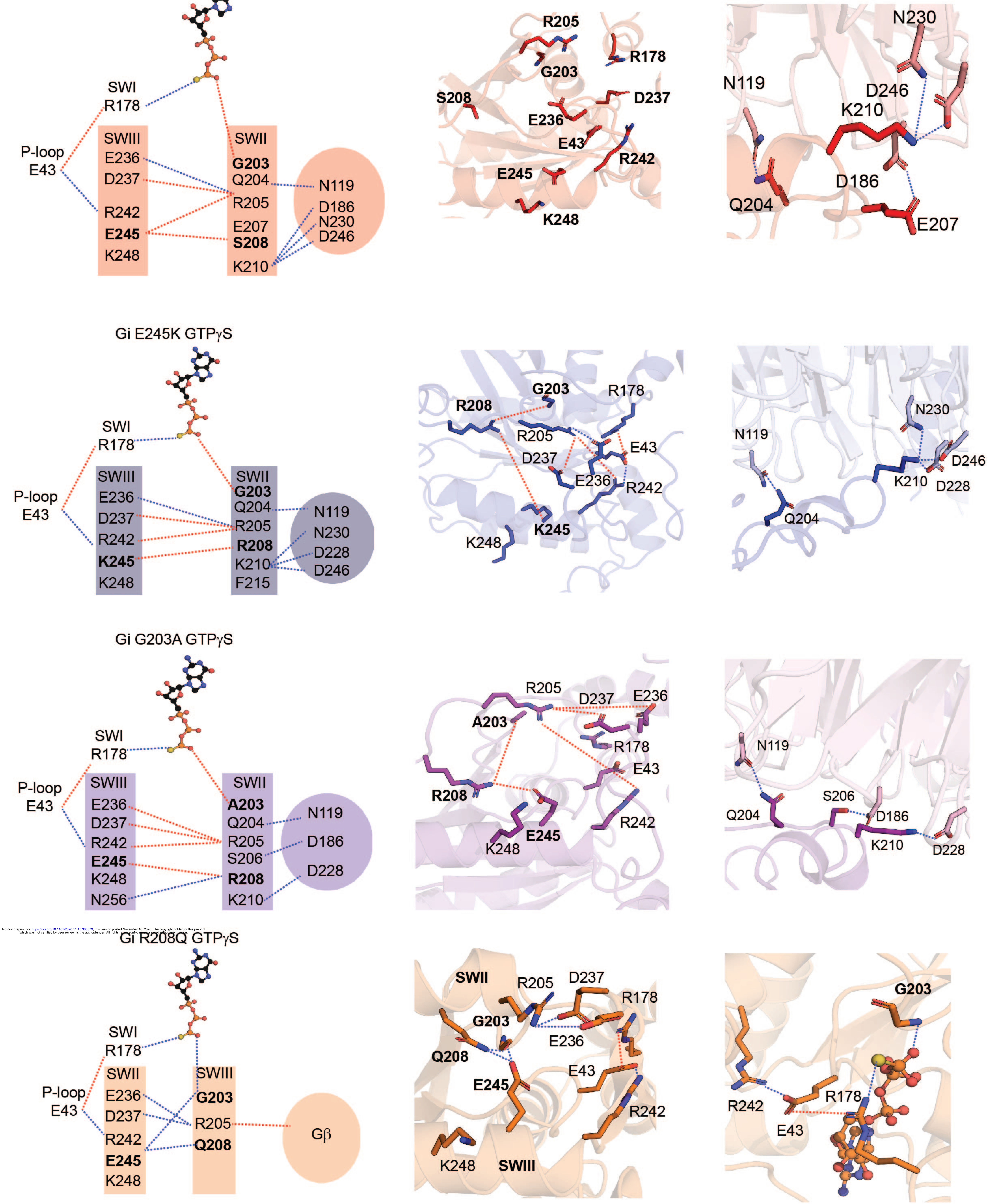
D Gs R231S GTP $\gamma$ S

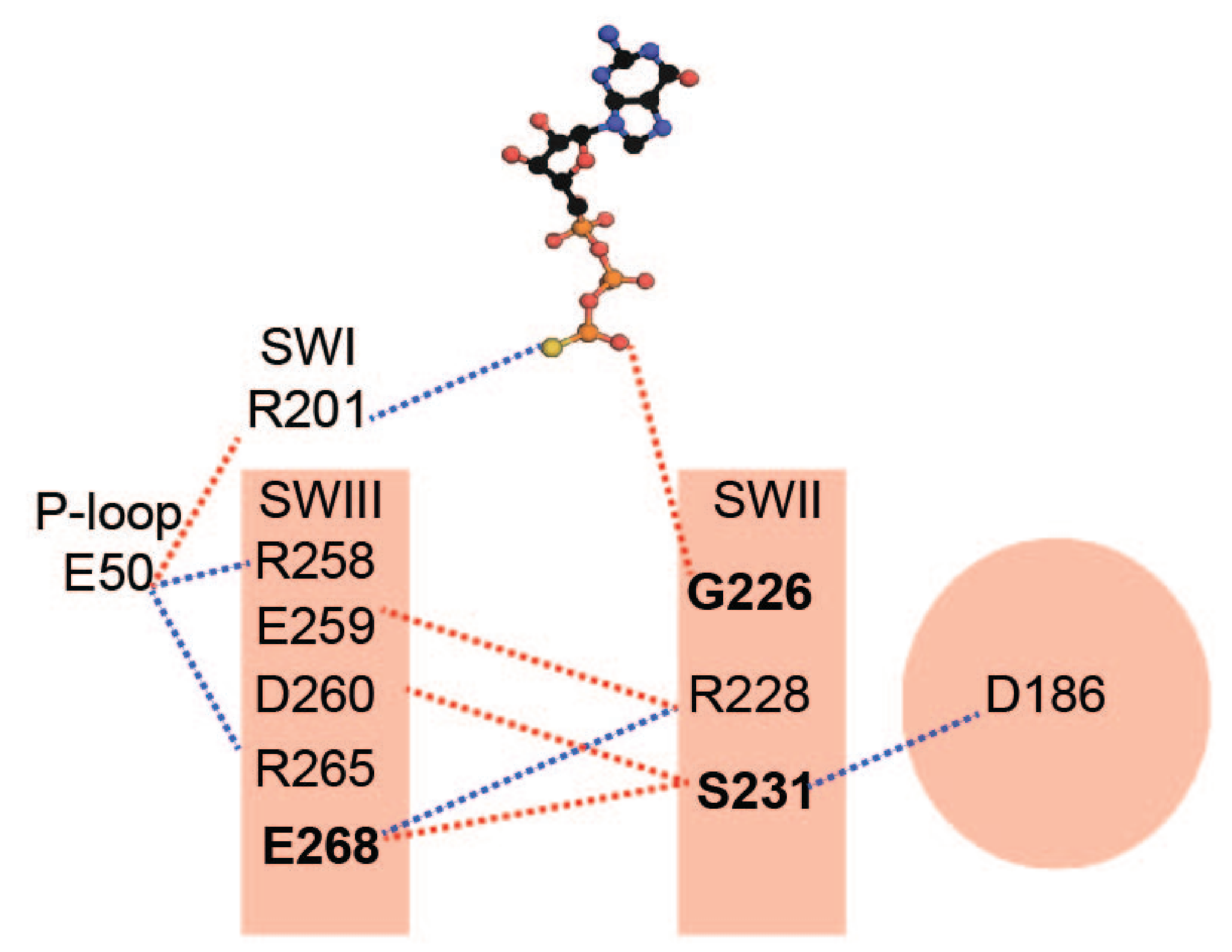

Gs E268K GTP $\gamma$ S
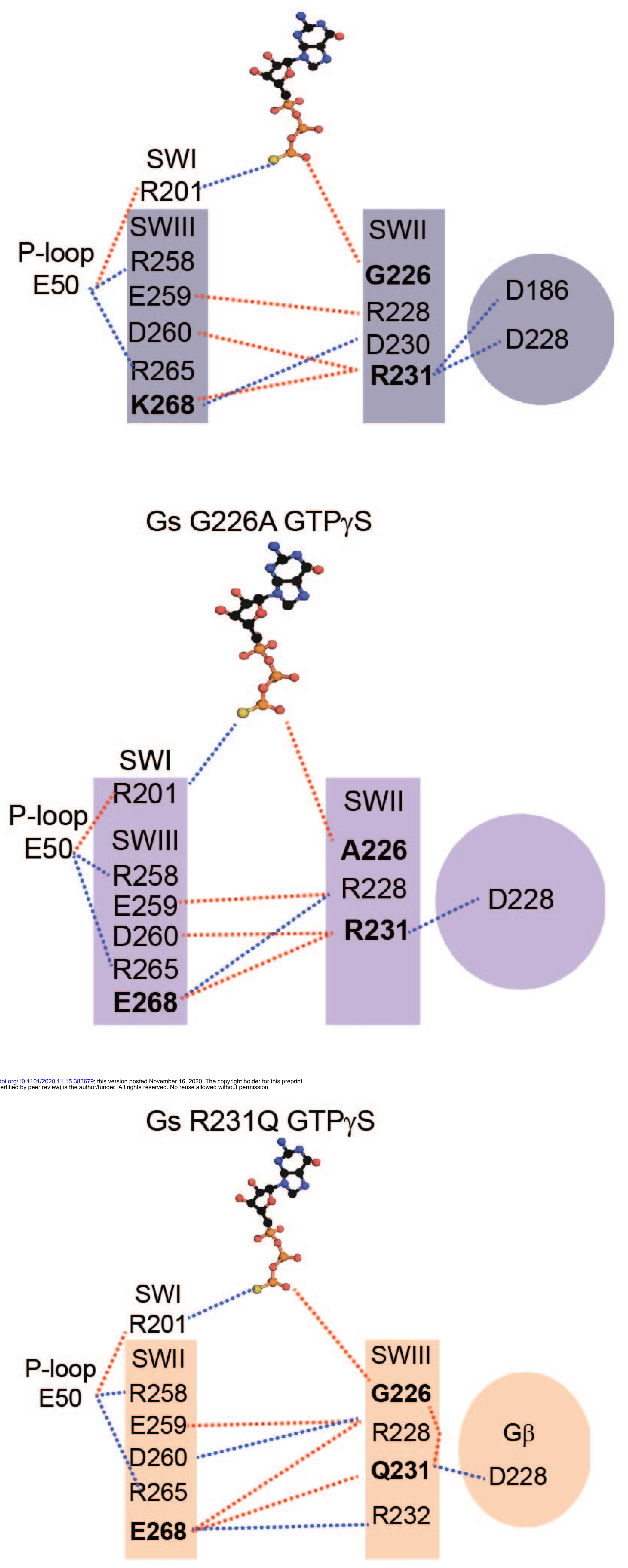
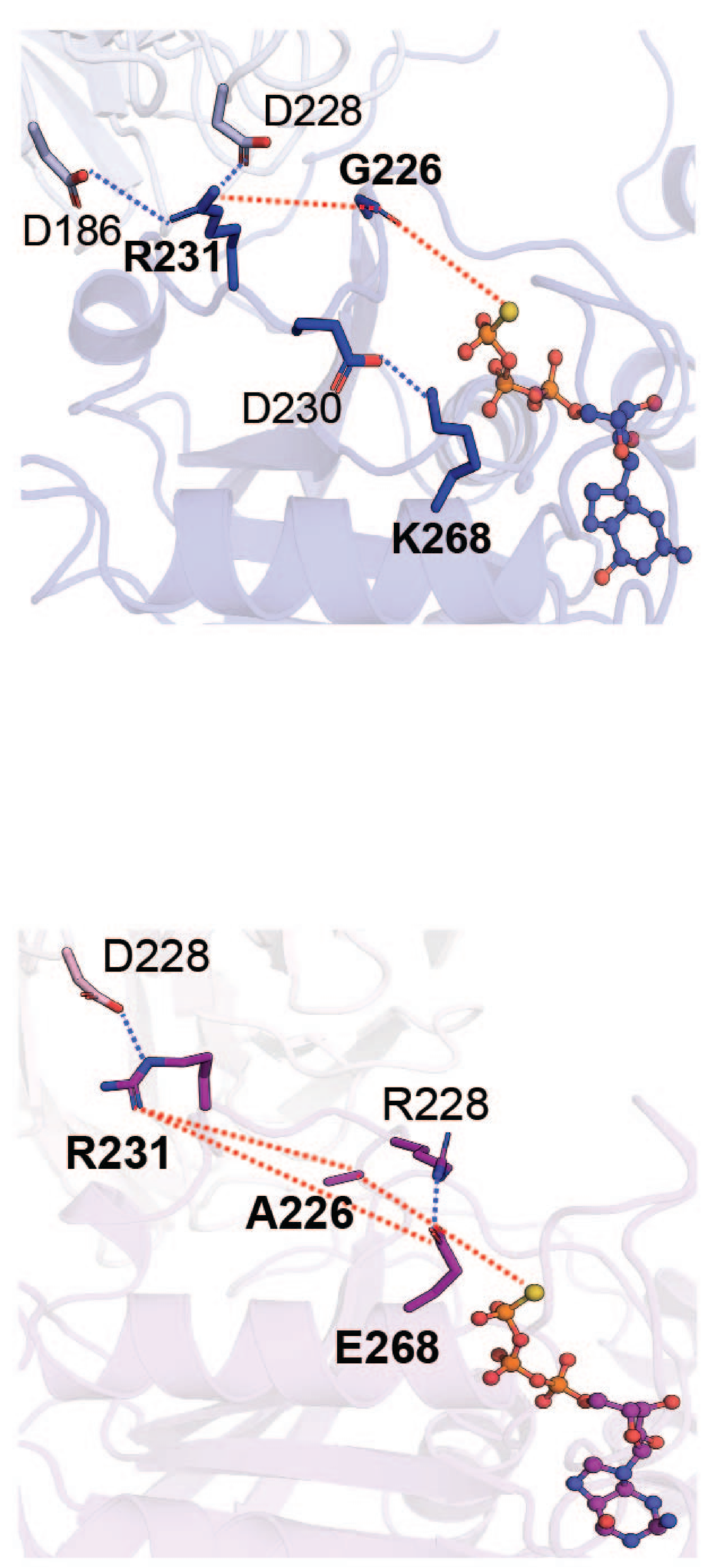

SWII-SWIII Interface
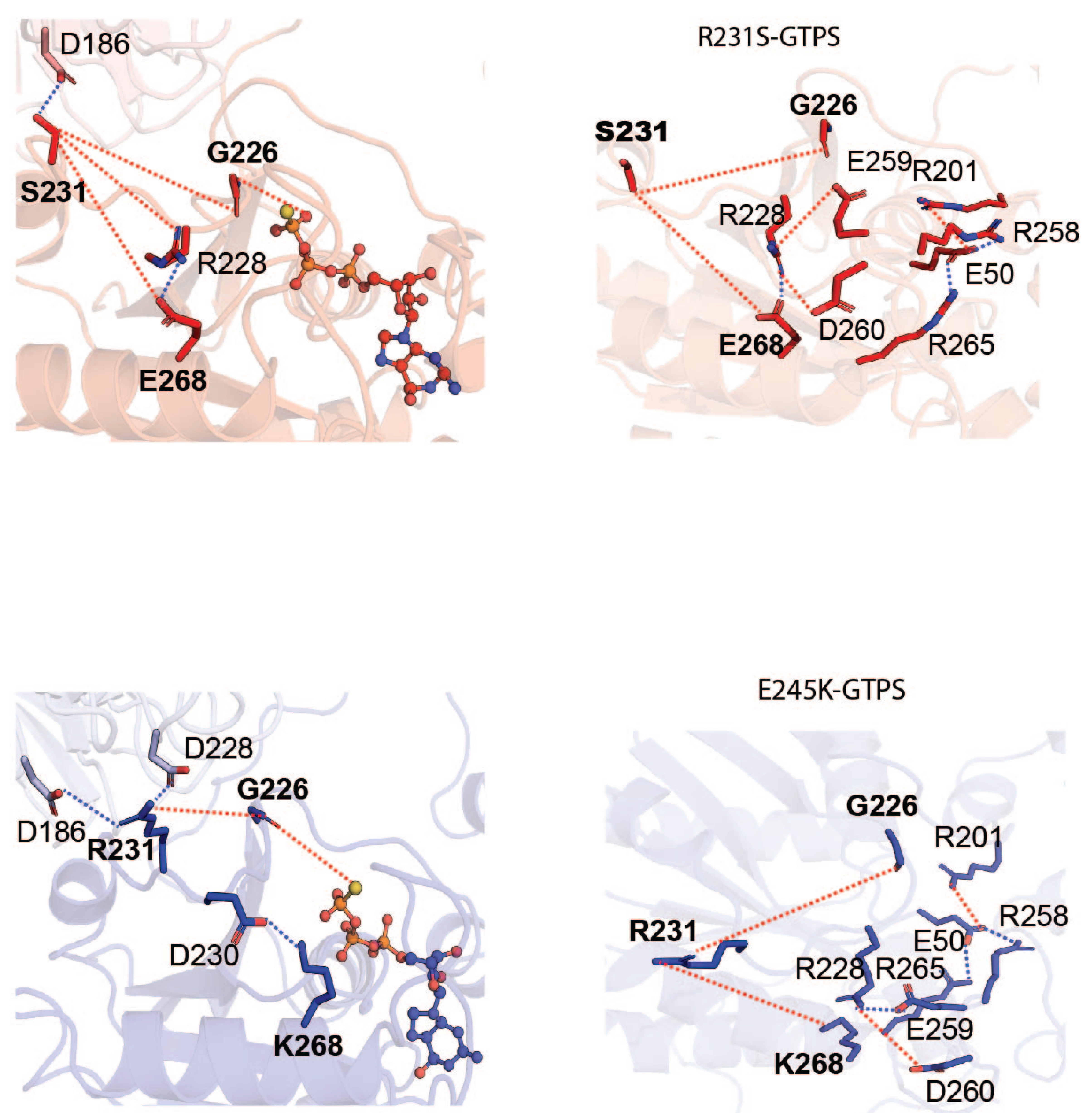

G226A-GTPS
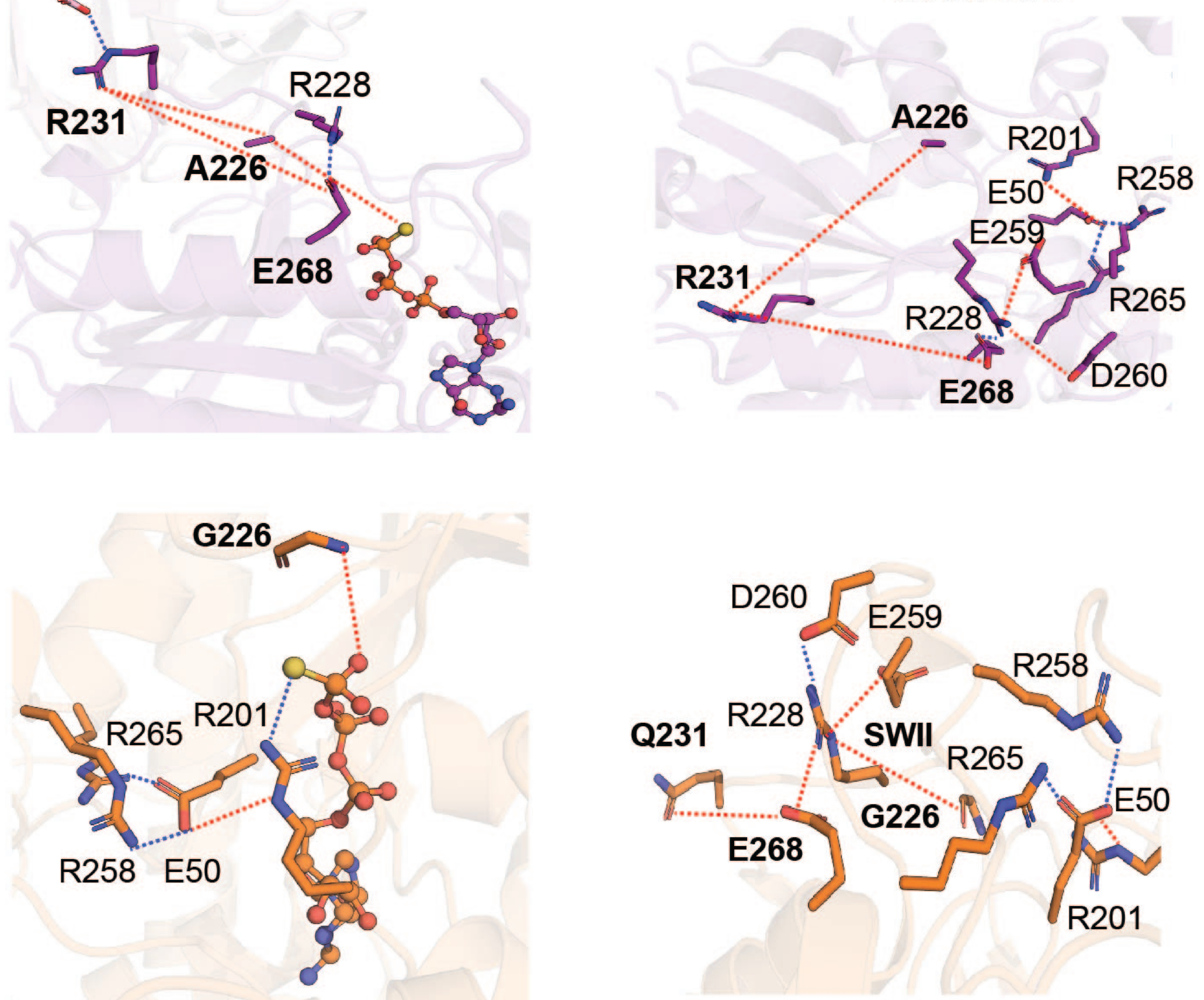
A
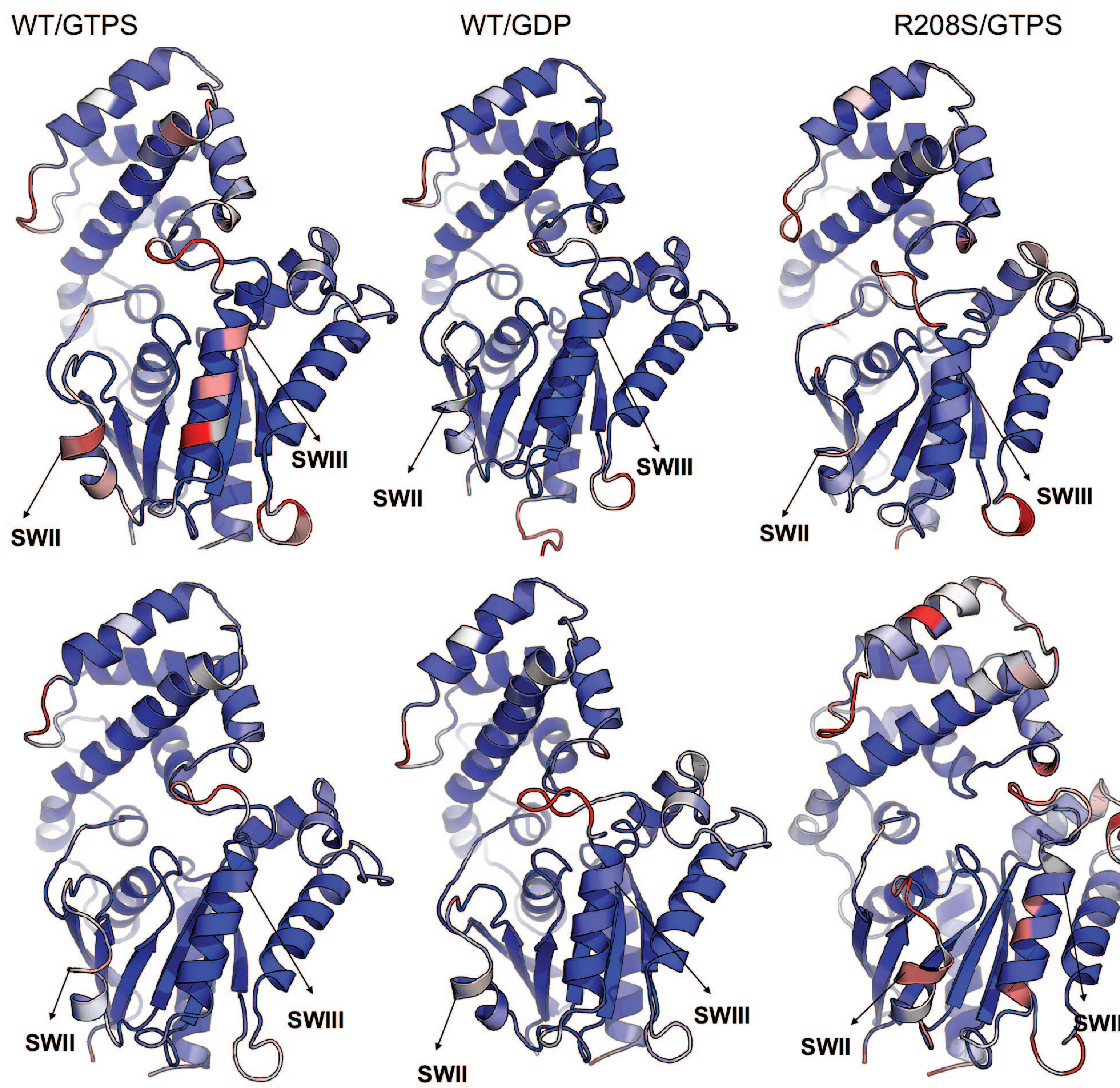

E245K/GTPS
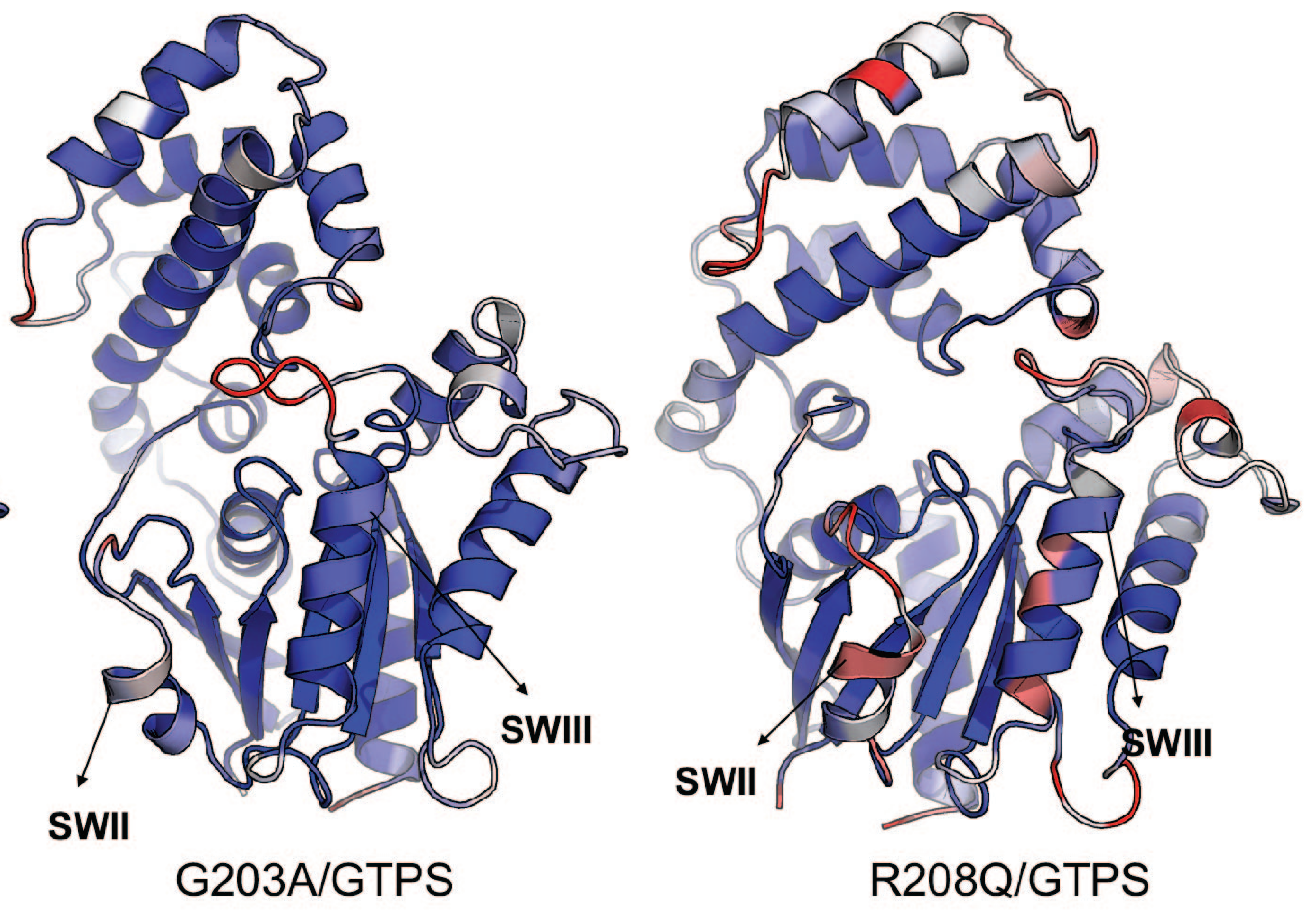

B

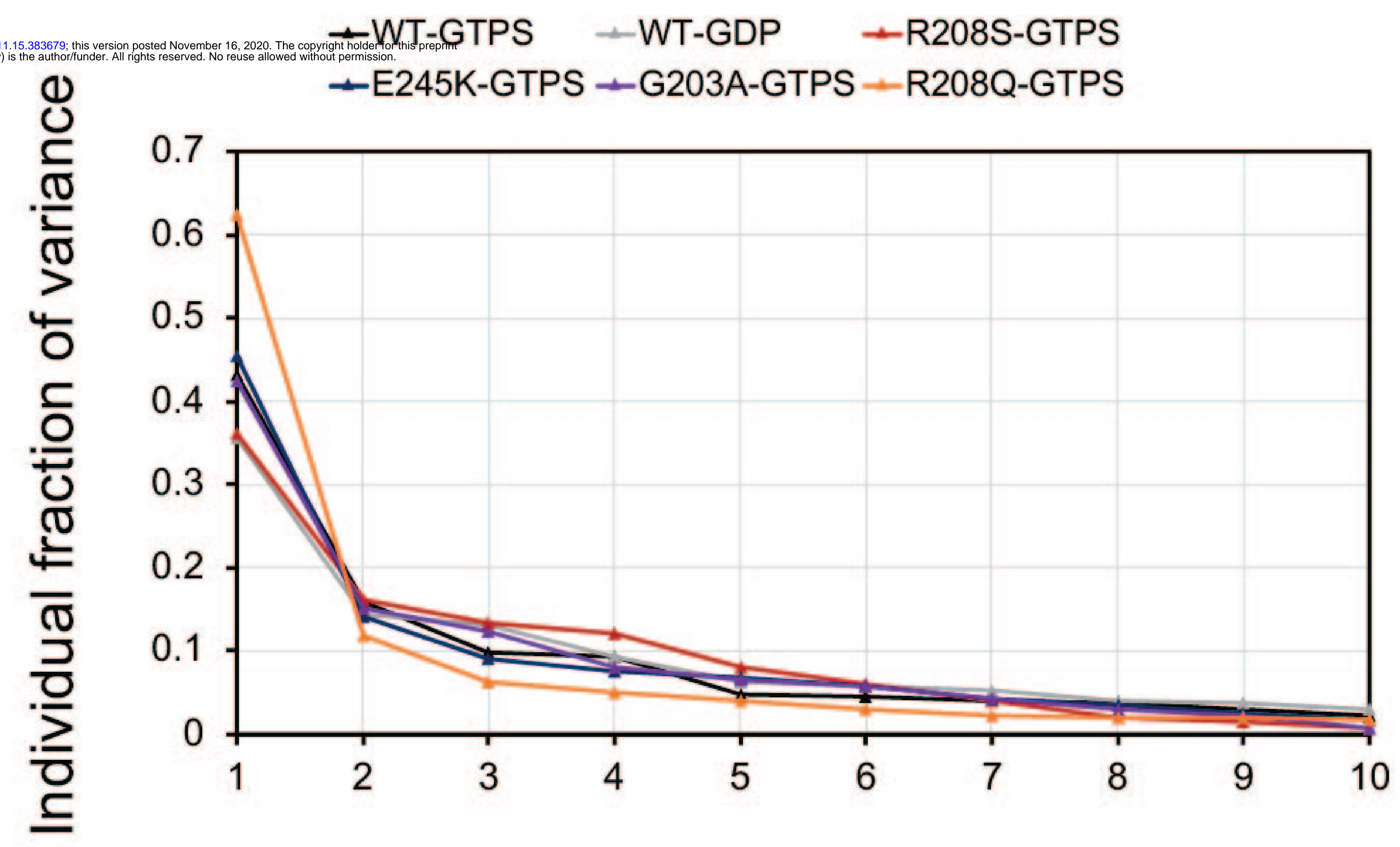

Eigenvector index
C

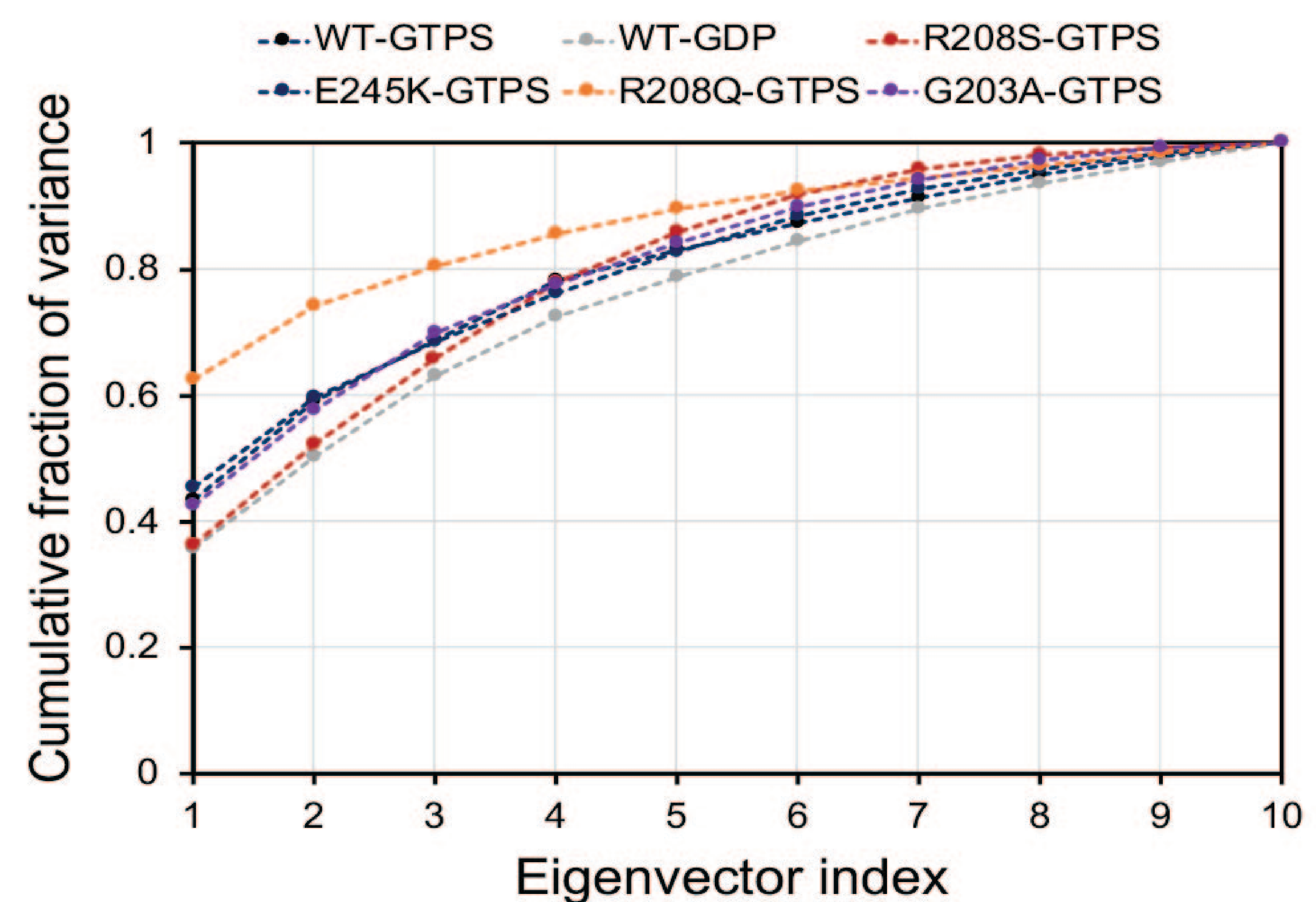




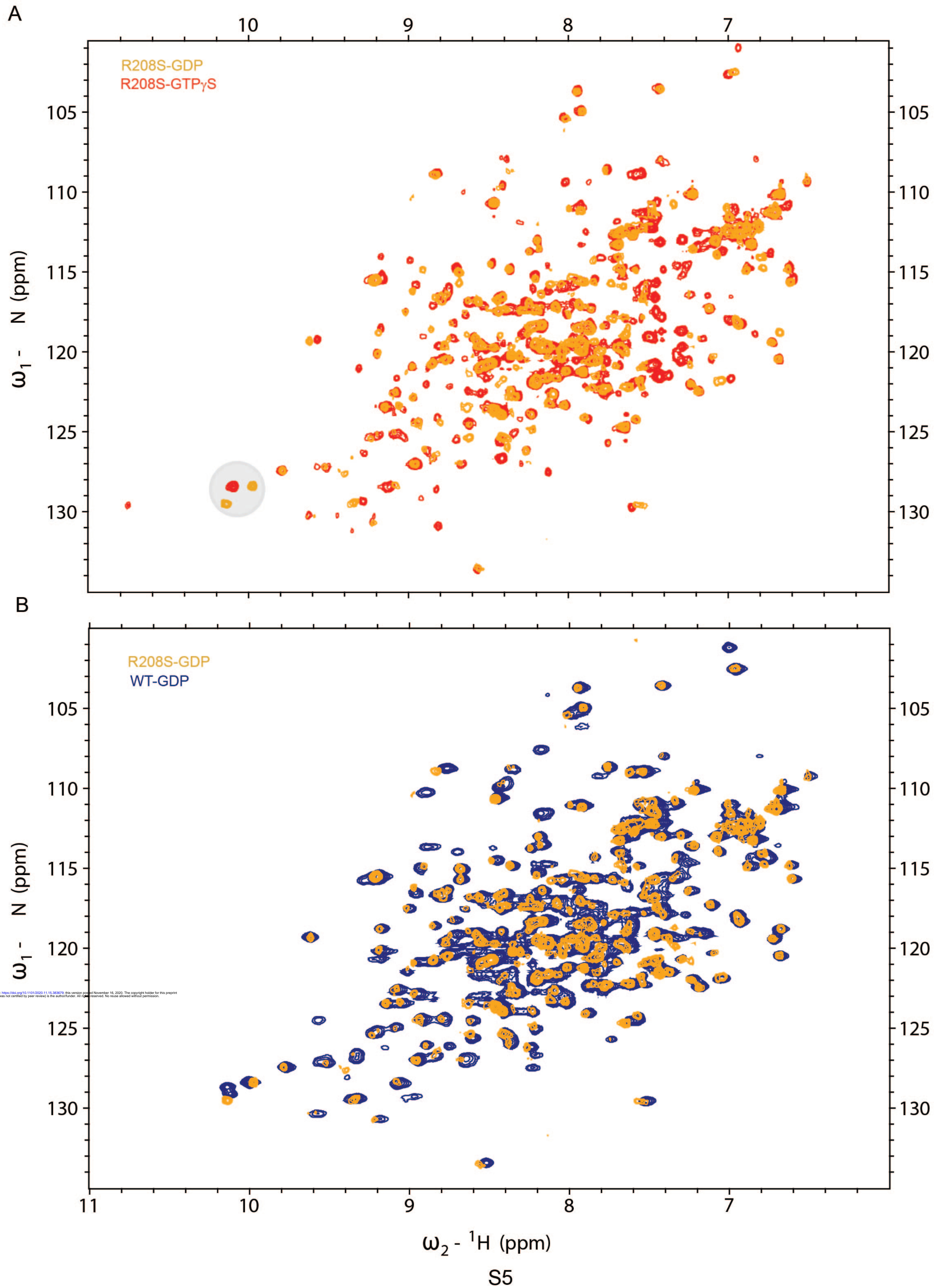

